\title{
Phosphine as a Biosignature Gas in Exoplanet Atmospheres
}

\author{
Clara Sousa-Silva, ${ }^{1,2}$ Sara Seager, ${ }^{1-3}$ Sukrit Ranjan,, ${ }^{1,4}$ Janusz Jurand Petkowski, ${ }^{1}$ Zhuchang Zhan, \\ Renyu $\mathrm{Hu}^{5,6}$ and William Bains ${ }^{7}$
}

\begin{abstract}
A long-term goal of exoplanet studies is the identification and detection of biosignature gases. Beyond the most discussed biosignature gas $\mathrm{O}_{2}$, only a handful of gases have been considered in detail. In this study, we evaluate phosphine $\left(\mathrm{PH}_{3}\right)$. On Earth, $\mathrm{PH}_{3}$ is associated with anaerobic ecosystems, and as such, it is a potential biosignature gas in anoxic exoplanets. We simulate the atmospheres of habitable terrestrial planets with $\mathrm{CO}_{2}$ - and $\mathrm{H}_{2}$-dominated atmospheres and find that $\mathrm{PH}_{3}$ can accumulate to detectable concentrations on planets with surface production fluxes of $10^{10}$ to $10^{14} \mathrm{~cm}^{-2} \mathrm{~s}^{-1}$ (corresponding to surface concentrations of $10 \mathrm{~s}$ of ppb to 100s of ppm), depending on atmospheric composition and ultraviolet (UV) irradiation. While high, the surface flux values are comparable to the global terrestrial production rate of methane or $\mathrm{CH}_{4}\left(10^{11} \mathrm{~cm}^{-2} \mathrm{~s}^{-1}\right)$ and below the maximum local terrestrial $\mathrm{PH}_{3}$ production rate $\left(10^{14} \mathrm{~cm}^{-2} \mathrm{~s}^{-1}\right)$. As with other gases, $\mathrm{PH}_{3}$ can more readily accumulate on low-UV planets, for example, planets orbiting quiet $\mathrm{M}$ dwarfs or with a photochemically generated UV shield. $\mathrm{PH}_{3}$ has three strong spectral features such that in any atmosphere scenario one of the three will be unique compared with other dominant spectroscopic molecules. Phosphine's weakness as a biosignature gas is its high reactivity, requiring high outgassing rates for detectability. We calculate that tens of hours of JWST (James Webb Space Telescope) time are required for a potential detection of $\mathrm{PH}_{3}$. Yet, because $\mathrm{PH}_{3}$ is spectrally active in the same wavelength regions as other atmospherically important molecules (such as $\mathrm{H}_{2} \mathrm{O}$ and $\mathrm{CH}_{4}$ ), searches for $\mathrm{PH}_{3}$ can be carried out at no additional observational cost to searches for other molecular species relevant to characterizing exoplanet habitability. Phosphine is a promising biosignature gas, as it has no known abiotic false positives on terrestrial planets from any source that could generate the high fluxes required for detection. Key Words: Phosphine-BiosignatureExoplanet-Atmospheric gases-Spectroscopy-Anoxic environments. Astrobiology 20, 235-268.
\end{abstract}

\section{Introduction}

$\mathbf{L}$ IFE MAKES USE of thousands of volatile molecular species that could contribute toward a biosphere and its associated atmospheric spectrum. Some of these volatiles may accumulate in a planetary atmosphere and be remotely detectable; these are commonly called "biosignature gases."

Theoretical studies of biosignature gases have been recently heavily reviewed elsewhere (Seager et al., 2016; Grenfell, 2018; Kiang et al., 2018; Schwieterman et al., 2018).

Prominent biosignature gases on Earth are those that are both relatively abundant and spectroscopically active (primarily $\mathrm{O}_{2}$, its photochemical by-product $\mathrm{O}_{3}$, and also $\mathrm{CH}_{4}$ and $\mathrm{N}_{2} \mathrm{O}$ ). Other gases that are not prominent in Earth's atmosphere but might be prominent in exoplanet atmospheres have also been studied, for example, dimethyl sulfide, dimethyl disulfide, and $\mathrm{CH}_{3} \mathrm{Cl}$ (Pilcher, 2003; Segura et al., 2005; Domagal-Goldman et al., 2011).

The next generation telescopes will open the era of the study of rocky exoplanet atmospheres. The James Webb Space Telescope (JWST, planned for launch in 2021) is the most capable for transmission spectra studies of a handful of the most suitable rocky planets transiting bright $\mathrm{M}$ dwarf stars (Gardner et al., 2006), whereas ESAs Atmospheric Remotesensing Infrared Exoplanet Large-survey (ARIEL, planned for launch in 2028) may be able to detect atmospheric components

Departments of ${ }^{1}$ Earth, Atmospheric, and Planetary Sciences, ${ }^{2}$ Physics, and ${ }^{3}$ Aeronautics and Astronautics, Massachusetts Institute of Technology (MIT), Cambridge, Massachusetts.

${ }_{5}^{4}$ SCOL Postdoctoral Fellow

${ }^{5}$ Jet Propulsion Laboratory, California Institute of Technology, Pasadena, California.

${ }^{6}$ Division of Geological and Planetary Sciences, California Institute of Technology, Pasadena, California.

${ }^{7}$ Rufus Scientific, Royston, United Kingdom. 
on super-Earths around the smallest $\mathrm{M}$ dwarf stars (Pascale et al., 2018). Large ground-based telescopes now under construction, that is, Giant Magellan Telescope, Extremely Large Telescope, and Thirty Meter Telescope (Johns et al., 2012; Tamai and Spyromilio, 2014; Skidmore et al., 2015), can also reach $\mathrm{M}$ dwarf star rocky planets by direct imaging, with the right instrumentation.

To the best of our knowledge, phosphine $\left(\mathrm{PH}_{3}\right)$ has not yet been evaluated as a biosignature gas. In Earth's atmosphere, $\mathrm{PH}_{3}$ is a trace gas. It is possible, however, that biospheres on other planets could accumulate significant detectable $\mathrm{PH}_{3}$ levels. In particular, anoxic biospheres where life would not be heavily dependent on oxygen could produce $\mathrm{PH}_{3}$ in significantly higher quantities than on modern Earth (Bains et al., 2019b).

Astronomical observations find that phosphine is spectroscopically active and present in stellar atmospheres (namely carbon stars) and in the giant planet atmospheres of Jupiter and Saturn (Bregman et al., 1975; Tarrago et al., 1992; Agúndez et al., 2014). In T dwarfs and giant planets, $\mathrm{PH}_{3}$ is expected to contain the entirety of the atmospheres' phosphorus in the deep atmosphere layers (Visscher et al., 2006), where it is sufficiently hot for $\mathrm{PH}_{3}$ formation to be thermodynamically favored.

In both Jupiter and Saturn, phosphine is found on the high observable layers at abundances (4.8 and $15.9 \mathrm{ppm}$, respectively) several orders of magnitude higher than those predicted by thermodynamic equilibrium (Fletcher et al., 2009). This overabundance of $\mathrm{PH}_{3}$ occurs because chemical equilibrium timescales are long when compared with convective timescales (Noll and Marley, 1997). $\mathrm{PH}_{3}$ forms in the hotter deep layers of the atmosphere (temperatures $\gtrsim$ $800 \mathrm{~K}$ ) and is mixed upward, so that the $\mathrm{PH}_{3}$ inventory at the cloud-top is replenished. In every astronomical body where phosphine has been detected thus far, other than on Earth, there are regions with high enough temperatures for $\mathrm{PH}_{3}$ to be the thermodynamically favored phosphorus species.

It has been postulated that elemental phosphorus species originating from the photolysis of phosphine are responsible for the red coloring of Jupiter's red spot and other Jovian chromophores (Prinn and Lewis, 1975), although this hypothesis has not achieved wide community acceptance (Noy et al., 1981; Kim, 1996). For a review of chemical species that are current candidates for the chromophores of Jupiter, see Carlson et al. (2016) and references therein.

Phosphine has not been detected in the observable layers of ice giants, such as Uranus and Neptune (Burgdorf et al., 2004; Moreno et al., 2009), despite these planets having sufficiently hot layers to produce $\mathrm{PH}_{3}$ and strong convection currents, which could transport $\mathrm{PH}_{3}$ to observable altitudes. Observations put the $\mathrm{P} / \mathrm{H}$ abundance in Uranus and Neptune at an upper limit of $<0.1$ solar $\mathrm{P} / \mathrm{H}$, which is significantly lower than expected (Teanby et al., 2019).

In this work, we critically assess phosphine as a biosignature gas. We first summarize in what circumstances phosphine is generated by life on Earth (Sections 2.1 and 2.2). We next review the known destruction mechanisms for $\mathrm{PH}_{3}$ (Section 2.3) and describe our inputs and methods for the assessment of the detectability of $\mathrm{PH}_{3}$ in a variety of planetary scenarios (Section 3). We then present our results (Section 4): here, we first calculate surface fluxes and associated atmospheric abundances required for the remote spectroscopic detection of $\mathrm{PH}_{3}$ in transmission and emission spectra (Section 4.1). We then highlight the properties of the $\mathrm{PH}_{3}$ spectrum that help distinguish it from other molecules (Section 4.2). Next, we present thermodynamic calculations that show that $\mathrm{PH}_{3}$, in temperate planets, has no substantial false positives as a biosignature gas (Section 4.3). We conclude with a discussion of our results (Section 5).

\section{Phosphine Sources and Sinks}

On Earth, phosphine is associated with biological production in anaerobic environments and anthropogenic production via a multitude of industrial processes. $\mathrm{PH}_{3}$ has low mean production rates on Earth, but it is a mobile gas and is found globally, albeit in trace amounts, in the atmosphere. Below, we summarize the known emissions of $\mathrm{PH}_{3}$ on Earth (Section 2.1), phosphine's association with life (Section 2.2), and its known destruction mechanisms (Section 2.3).

\subsection{Phosphine emissions on Earth}

Phosphine is a ubiquitous trace component of the atmosphere on modern Earth (Morton and Edwards, 2005). About $10 \%$ of the phosphorus in the atmosphere is $\mathrm{PH}_{3}$; the major phosphorus form is phosphate, mostly as phosphoric acid (Elm et al., 2017). Although $\mathrm{PH}_{3}$ is found everywhere in Earth's atmosphere, its atmospheric abundance is widely variant, with high concentration regions sometimes having more $\mathrm{PH}_{3}$ than low concentration areas by a factor of 10,000 (Pasek et al., 2014).

Phosphine has been found worldwide in the lower troposphere of Earth in the ppq to ppb range in daytime, with higher nighttime concentrations than at daytime (due to inhibited ultraviolet [UV]-induced oxidation) (Gassmann, 1994; Gassmann et al., 1996; Glindemann et al., 1996b, 2003; Ji-ang et al., 1999; Han et al., 2000; Zhu et al., 2006a, 2007a, 2007b; Li et al., 2009; Hong et al., 2010a; Zhang et al., 2010). In the high troposphere, $\mathrm{PH}_{3}$ was found at a peak of $7 \mathrm{ppt}$ during daylight (Glindemann et al., 2003; Han et al., 2011b). This implies that sunlight does not lead to complete destruction of $\mathrm{PH}_{3}$, unlike previous suggestions (Glindemann et al., 2003; Han et al., 2011b). A sample of locally measured gaseous $\mathrm{PH}_{3}$ concentrations in a variety of environments on Earth, ranging from $\mathrm{ppq}$ to $\mathrm{ppb}\left(\mathrm{ng} / \mathrm{m}^{3}\right.$ to $\left.\mu \mathrm{g} / \mathrm{m}^{3}\right)$, can be found in Fig. 1 .

On Earth, a significant source of phosphine emissions is anthropogenic activity. Because of its broad toxicity to aerobic organisms, ${ }^{*} \mathrm{PH}_{3}$ is widely used in the agricultural industry as a rodenticide and insecticide (Devai et al., 1988; Bingham, 2001; Glindemann et al., 2005; Perkins et al., 2015; Chen et al., 2017). $\mathrm{PH}_{3}$ is also used commercially, for example, as a doping agent (Budavari et al., 1996). However, $\mathrm{PH}_{3}$ emissions linked to biological activity are believed to form the majority of atmospheric $\mathrm{PH}_{3}$ (Glindemann et al., 2005; Morton and Edwards, 2005). Evidence for the association of $\mathrm{PH}_{3}$ with anaerobic biology is presented in Section 2.2.

\subsection{Biological production of phosphine}

All life on Earth relies on phosphorous compounds. The biological phosphorus cycle is heavily, but not exclusively,

*Exposure to phosphine abundances of 400 ppm results in a quick death (Fluck, 1973). 


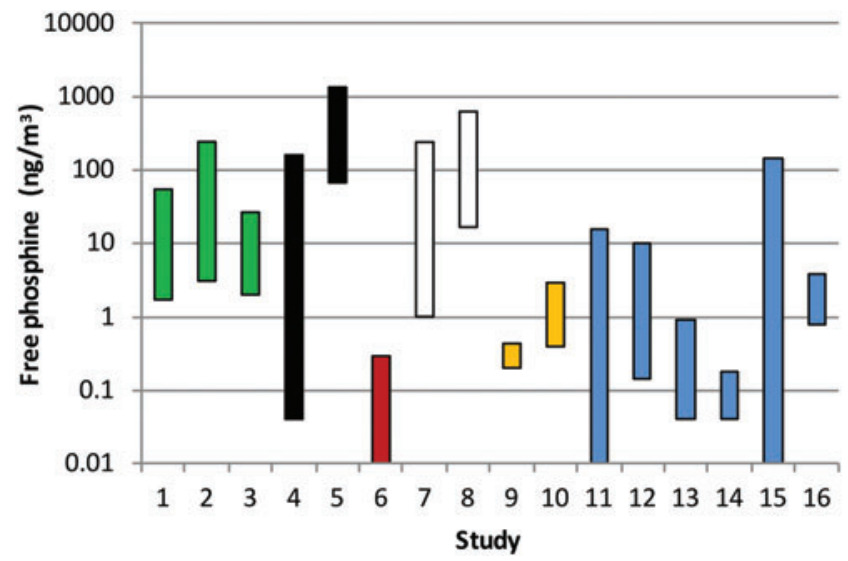

FIG. 1. Measurements of phosphine concentrations in Earth's atmosphere. Study number shown in $x$ axis (references below) and $y$ axis showing the span of locally measured concentration of gaseous $\mathrm{PH}_{3}$ in units of $\mathrm{ng} / \mathrm{m}^{3}$, with maximum values of 600.2 and $1259 \mathrm{ng} / \mathrm{m}^{3}$ (corresponding to concentrations ranging between ppq and $\mathrm{ppb}$ ). Green bars: marshlands and paddy fields. Black bars: industrial environments. Red bar: Namibia (rural environment). White bars: Arctic and Antarctic environments. Yellow bars: upper troposphere. Blue bars: oceanic samples (coastal and open ocean). References for studies shown: (1) Han et al. (2011a); (2) Han et al. (2000); (3) Niu et al. (2013); (4) Glindemann et al. (1996a); (5) Zhang et al. (2010); (6) Glindemann et al. (1996a); (7) Zhu et al. (2007a, 2007b); (8) Zhang et al. (2010); (9, 10) Glindemann et al. (2003); (11) Li et al. (2009); (12) Zhu et al. (2007a, 2007b); (13) Gassmann et al. (1996); (14) Glindemann et al. (2003); (15) Geng et al. (2005), Han et al. (2011b); (16) Hong et al. (2010a). We do not include measurements of "MatrixBound Phosphine" (MBP), material that releases $\mathrm{PH}_{3}$ when a matrix is treated with high temperatures and strong acid or alkali. Figure adapted from the work of Bains et al. (2019a). Please see Bains et al. (2019a) for more details on MBP and environmental $\mathrm{PH}_{3}$ production. Gaseous $\mathrm{PH}_{3}$ is found in multiple altitudes in Earth's atmosphere above a wide variety of environments, in concentrations ranging from ppq to ppb. MBP, Matrix-Bound Phosphine; $\mathrm{PH}_{3}$, phosphine. Color images are available online.

reliant on phosphates. Other, less oxidized, phosphoruscontaining molecules also play a crucial role in the phosphorus cycle. The exact role of phosphine in the Earth's global phosphorus cycle is not yet fully known. It is, however, likely that, similar to other reduced phosphorus species, $\mathrm{PH}_{3}$ also has an important role in the global cycling of this essential element. For more details on the role of phosphines in the Earth's phosphorus cycle, please see Appendix E.

Biological phosphine production is associated with microbial activity in environments that are strictly anoxic (lacking oxygen). This finding is in alignment with the fact that the toxicity of $\mathrm{PH}_{3}$ is intrinsically linked to its interference with $\mathrm{O}_{2}$-dependent metabolism (Bains et al., 2019b).

The argument that phosphine is associated with anaerobic life is strengthened by its detection in a wide variety of ecosystems with anoxic niches, including above penguin colonies, rich in bird guano, where it reaches abundances of 300 ppt (Ji-ang et al., 1999; Zhu et al., 2006a, 2007a; Li et al., 2009; Hong et al., 2010a); animal intestinal tracts, flatus, and feces ${ }^{\dagger}$ (Gassmann and Glindemann, 1993; Eismann et al., 1997a; Chughtai and Pridham, 1998; Zhu et al., 2006a, 2006b, 2014); paddy fields (Han et al., 2011a; Chen et al., 2017); rivers and lakes (Feng et al., 2008; Geng et al., 2010; Hong et al., 2010b; Han et al., 2011b; Ding et al., 2014); wetlands and marshlands (Devai and Delaune, 1995; Eismann et al., 1997b; Glindemann et al., 1996b); and landfills and sludges (Roels and Verstraete, 2004; Ding et al., 2005a, 2005b). Several studies have also reported the production of $\mathrm{PH}_{3}$ from mixed bacterial cultures in the laboratory (Rutishauser and Bachofen, 1999; Jenkins et al., 2000; Liu et al., 2008); in one case, bacteria turning half of the phosphorus in the culture medium $(\sim 180 \mathrm{mg} / \mathrm{L})$ into $\mathrm{PH}_{3}$ in 56 days (Devai et al., 1988).

Despite a large body of robust circumstantial evidence for the production of phosphine by life, the exact mechanisms for biologically associated production of $\mathrm{PH}_{3}$ are still debated. Recent work postulates that $\mathrm{PH}_{3}$ production may be associated with the microbial tricarboxylic acid cycle of Enterobacteriaceae (Fan et al., 2020), but the exact metabolic pathway leading to $\mathrm{PH}_{3}$ production in anaerobic organisms remains unknown. However, we note that the absence of a known enzymatic mechanism is not evidence for the absence of biological production. The synthetic pathways for most of life's natural products are not known, and yet their origin is widely accepted to be biological because of the implausibility of their abiotic synthesis, their obligate association with life, and their chemical similarity to other biological products. For example, a recently published, manually curated, database of natural molecules produced by life on Earth contains 220,000 unique molecules of biological origin, produced by thousands of species (Petkowski et al., 2019a), whereas the number of known, experimentally elucidated, metabolic pathways from organisms belonging to all three domains of life is only $\sim 2720$ (Caspi et al., 2017). Further examples of the complexities in discovering metabolic pathways for molecules associated with biological activity are provided in Appendix E.

There are two proposed explanations for the production of phosphine in anoxic ecosystems [reviewed in Glindemann et al. (1998), Roels and Verstraete (2001), Roels and Verstraete (2004), Roels et al. (2005), Bains et al. (2019a, 2019b)]:

(1) $\mathrm{PH}_{3}$ is directly produced by anaerobic bacteria from environmental phosphorus.

(2) $\mathrm{PH}_{3}$ is indirectly produced by anaerobic bacteria. Anoxic fermentation of organic matter by anaerobic bacteria results in acid products; these acid products, in turn, could react with inorganic metal phosphides, like those present as trace elements in scrap metal, resulting in phosphine generation.

Of the two proposed explanations for biologically associated production of phosphine, we argue that the direct production as a result of metabolic activity of anaerobic bacteria is the most likely. Our reasoning is based on two lines of evidence:

\footnotetext{
${ }^{\dagger}$ We note that animal guts are anaerobic, even in small animals.

In agricultural wetlands, both industrial and biological sources of $\mathrm{PH}_{3}$ are plausible.
} 
(a) $\mathrm{PH}_{3}$ has been detected in significant amounts in bacterial cultures in controlled laboratory experiments, where no metal phosphides were present, making the indirect acid-dependent production of $\mathrm{PH}_{3}$ an unlikely scenario (Devai et al., 1988; Glindemann et al., 1996b; Jenkins et al., 2000; Schink and Friedrich, 2000; Ding et al., 2005a, 2005b; Liu et al., 2008).

(b) Several independent studies found that $\mathrm{PH}_{3}$ was detected in feces from evolutionarily distant animals, inhabiting diverse environments, for example, insects, birds, and mammals (including humans) (Gassmann and Glindemann, 1993; Chughtai and Pridham, 1998; Zhu et al., 2014). It is implausible that there is a significant presence of contaminant metal phosphides in the guts of all the animals, which would be required for an indirect acid-dependent production of $\mathrm{PH}_{3}$.

We end this introduction to the biological association of phosphine by noting that thermochemical studies on the feasibility of the production of $\mathrm{PH}_{3}$ in temperate environments have found no plausible thermodynamically favored abiotic pathways, and as such, $\mathrm{PH}_{3}$ has no substantial false positives for life (see Section 4.3 and Appendix C) (Bains et al., 2019a). Conversely, the production of $\mathrm{PH}_{3}$ under anoxic conditions by living systems can be thermochemically favorable (Bains et al., 2019a), and biological functions that are accomplished through energy consuming reactions are not uncommon (Bains et al., 2019b). $\mathrm{PH}_{3}$ could be used by life to perform complex functions that would warrant an energetic investment, such as signaling or a defense mechanism (Bains et al., 2019b). For more information about $\mathrm{PH}_{3}$ in the context of terrestrial biology and the thermodynamic feasibility of $\mathrm{PH}_{3}$ production by life, see Bains et al. (2019a, 2019b) and Appendices C-E.

\subsection{Phosphine chemistry in the atmosphere}

Within an atmosphere, phosphine is destroyed by the radicals $\mathrm{O}, \mathrm{H}$, and $\mathrm{OH}$ in reactions, which are thought to be first order with respect to its reactants and second order overall. $\mathrm{PH}_{3}$ can also be regenerated by reaction of $\mathrm{PH}_{2}$ with $\mathrm{H}$ and directly photolyzed by UV radiation. These processes are summarized below and discussed in Section 5.2.

Reaction rate constants are expressed via the Arrhenius equation:

$$
k=A e^{-E / R T},
$$

where $k$ is the reaction rate constant in units of $\mathrm{cm}^{3} \mathrm{~s}^{-1}, A$ is a constant in units of $\mathrm{cm}^{3} \mathrm{~s}^{-1}, E$ is the activation energy in units of $\mathrm{J} \mathrm{mol}^{-1}, R$ is the gas constant in units of $\mathrm{J} \mathrm{mol}^{-1} \mathrm{~K}^{-1}$, and $T$ is temperature in $\mathrm{K}$.

2.3.1. Destruction by $\mathrm{OH}$ radicals. Oxidation with $\mathrm{OH}$ radicals is thought to be the main sink for phosphine in Earth's atmosphere via the reaction (Cao et al., 2000; Glindemann et al., 2005; Elm et al., 2017):

$$
\begin{aligned}
& \mathrm{PH}_{3}+\mathrm{OH} \rightarrow \mathrm{H}_{2} \mathrm{O}+\mathrm{PH}_{2} \\
& \mathrm{PH}_{2}+[\mathrm{O} \text { species }] \rightarrow \rightarrow \text { products (including } \mathrm{H}_{3} \mathrm{PO}_{4} \text { ). }
\end{aligned}
$$

For this reaction, $A=2.71 \times 10^{-11} \mathrm{~cm}^{3} \mathrm{~s}^{-1}, E=1.29 \mathrm{~kJ}$ $\mathrm{mol}^{-1}$, corresponding to $k_{\mathrm{PH}_{3}, \mathrm{OH}}=2 \times 10^{-11} \mathrm{~cm}^{3} \mathrm{~s}^{-1}$ at $T=288$ $\mathrm{K}$ (Fritz et al., 1982). The lifetime of $\mathrm{PH}_{3}$ due to $\mathrm{OH}$ reactions is calculated to be $28 \mathrm{~h}$ at night and $5 \mathrm{~h}$ in daytime, with the difference controlled by the concentration of UV-generated $\mathrm{OH}$ (Glindemann et al., 2003). The destruction of $\mathrm{PH}_{3}$ by $\mathrm{OH}$ in the atmosphere eventually leads to phosphoric acid, which in turn contributes to the soluble phosphates found in rainwater (Lewis et al., 1985; Elm et al., 2017).

2.3.2. Destruction by $\mathrm{O}$ radicals. Phosphine also reacts very rapidly with atomic oxygen (on Earth, generated by photolyzed ozone), with reaction:

$$
\mathrm{PH}_{3}+\mathrm{O} \rightarrow \longrightarrow \text { products (including } \mathrm{H}_{3} \mathrm{PO}_{4} \text { ). }
$$

For this reaction, $A=4.75 \times 10^{-11} \mathrm{~cm}^{3} \mathrm{~s}^{-1}, E \approx 0$, corresponding to $k_{\mathrm{PH}_{3}, \mathrm{O}}=5 \times 10^{-11} \mathrm{~cm}^{3} \mathrm{~s}^{-1}$ at $T=288 \mathrm{~K}$ (i.e., temperature-independent in 208-423 K, Nava and Stief, 1989). Because atomic oxygen is less abundant than $\mathrm{OH}$ in Earth's atmosphere and troposphere, $\mathrm{PH}_{3}$ destruction by $\mathrm{OH}$ is still the dominant route despite $\mathrm{PH}_{3}$ reacting with $\mathrm{O}$ at a higher rate. On Earth, $\mathrm{OH}$ radicals described above are partially generated from the interaction between $\mathrm{O}$ radicals and water vapor, and so the reactions that produce the two radical species are not happening in isolation (Jacob, 1999). In anoxic atmospheres, however, the main source of $\mathrm{OH}$ and $\mathrm{H}$ radicals is the photolysis of water vapor (Hu et al., 2012).

2.3.3. Destruction by $\mathrm{H}$ radicals. Phosphine can be destroyed by the $\mathrm{H}$ radical via the reaction:

$$
\mathrm{PH}_{3}+\mathrm{H} \rightarrow \mathrm{H}_{2}+\mathrm{PH}_{2} \text {. }
$$

For this reaction, $A=7.22 \times 10^{-11} \mathrm{~cm}^{3} \mathrm{~s}^{-1}, E=7.37 \mathrm{~kJ}$ $\mathrm{mol}^{-1}$, corresponding to $k_{\mathrm{PH}_{3}, \mathrm{H}}=3 \times 10^{-12} \mathrm{~cm}^{3} \mathrm{~s}^{-1}$ at $T=288$ $\mathrm{K}$ (Arthur and Cooper, 1997). The reaction of $\mathrm{PH}_{3}$ with the $\mathrm{H}$ radical is most relevant to $\mathrm{H}_{2}$-rich atmospheres (Seager et al., 2013b).

2.3.4. Recombination from $\mathrm{H}$ radicals. Phosphine can be regenerated by the radical recombination reaction:

$$
\mathrm{PH}_{2}+\mathrm{H} \rightarrow \mathrm{PH}_{3}
$$

With rate constant $k_{\mathrm{PH}_{2}, \mathrm{H}}=3.7 \times 10^{-10} \exp (-340 K / T) \mathrm{cm}^{3} \mathrm{~s}^{-1}$, corresponding to $k_{\mathrm{PH}_{2}, \mathrm{H}}=1.1 \times 10^{-10} \mathrm{~cm}^{3} \mathrm{~s}^{-1}$ at $T=288 \mathrm{~K}$ (Kaye and Strobel, 1984). If $\left[\mathrm{PH}_{2}\right]$ is high, this reaction can be a major reformation pathway for $\mathrm{PH}_{3}$ (see Section 5.2).

2.3.5. Destruction through UV radiation. UV radiation is thought to directly photolyze phosphine with unit quantum efficiency upon absorption of irradiation at wavelengths $\leq 230 \mathrm{~nm}$ (Visconti, 1981; Kaye and Strobel, 1984):

$$
\mathrm{PH}_{3}+h v \rightarrow \mathrm{PH}_{2}+\mathrm{H} .
$$

This photolysis reaction is not relevant on UV-shielded planets (e.g., modern Earth with its ozone layer) but could be relevant on anoxic planets where UV radiation may penetrate deeper into lower altitudes of the atmosphere.

Overall, phosphine is destroyed by UV irradiation, through both direct photolysis and reactions with UV-generated radical species. The daytime-nighttime $\mathrm{PH}_{3}$ concentration difference on Earth is large due to the generation of radicals by 
UV irradiation during the day and their comparative absence at night. However, $\mathrm{PH}_{3}$ has been detected at concentrations of up to $7 \mathrm{ppt}\left(2.45 \mathrm{ng} \mathrm{m}^{-3}\right)$ during daylight in Earth's high troposphere (Glindemann et al., 2003). $\mathrm{PH}_{3}$ accumulates in the dry upper troposphere on Earth because ozone attenuation of $\mathrm{UV}$ and lack of $\mathrm{OH}$-producing $\mathrm{H}_{2} \mathrm{O}$ result in low abundances of $\mathrm{OH}$ radicals, which slows the $\mathrm{PH}_{3}$ destruction and its return to the surface in the form of phosphates (Frank and Rippen, 1987; Glindemann et al., 2003).

2.3.6. Solubility and aerosol formation. Phosphine does not easily stick to aerosols and has very low water solubility (Fluck, 1973). $\mathrm{PH}_{3}$ is therefore a very mobile gas that is less likely to wash out and fall to the surface than other gases, such as hydrogen sulfide, methanethiol, and ammonia (Glindemann et al., 2003).

UV photolysis of phosphine in the presence of hydrocarbons could lead to the formation of complicated alkylphosphines (Guillemin et al., 1995, 1997). Atmospheres that are prone to high concentrations of hydrocarbon radicals, such as $\mathrm{H}_{2}$-rich atmospheres, could therefore lead to the creation of organophosphine hazes. The plausibility of organophosphine haze formation is discussed further in Section 5.2.

\section{Inputs and Methods for the Assessment of Detectability}

We have assessed the spectral distinguishability, atmospheric survival, and observational detectability of phosphine in anoxic exoplanets. In this section, we first describe the choice of molecular inputs used for our spectral analyses (Section 3.1). We then provide a brief outline of the photochemical method used to calculate the distribution of molecules throughout the atmosphere (Section 3.2). Finally, we outline the method and detectability criteria for the simulations of observational spectra (Section 3.3).

\subsection{Molecular inputs}

Molecular spectra can be represented in various forms to best serve as input for spectral representations and atmospheric models. For the comparison of phosphine with other major components of atmospheres (see Section 4.2), we have used cross sections calculated from the most complete spectra available. The $\mathrm{PH}_{3}$ molecular cross sections come from the recently variationally computed $\mathrm{PH}_{3}$ line list (Sousa-Silva et al., 2015) and the total internal partition function calculated in the work of Sousa-Silva et al. (2014). For all temperatures under $800 \mathrm{~K}$ (which includes all temperate environments), this $\mathrm{PH}_{3}$ line list represents a complete spectrum containing over 16 billion transitions between 7.5 million energy levels. Even at low temperatures, it is recommended that complete line lists are used for spectral simulations; complete line lists allow for more representative cross sections with improved band shapes when compared with experimental or calculated spectra at room temperature. The carbon dioxide line list is from HITEMP (Rothman et al., 2010). All other molecular cross sections are simulated by using complete, theoretically calculated, line lists from the ExoMol database (Yurchenko et al., 2011; Yurchenko and Tennyson, 2014; Sousa-Silva et al., 2015; Tennyson et al., 2016).
For the calculation of the transmission and thermal emission spectra of the model atmospheres (see Section 4.1), molecular opacities for phosphine are adopted from the ExoMol database (Sousa-Silva et al., 2015; Tennyson et al., 2016). For all other molecules, we used the HITEMP and the HITRAN 2016 databases (Rothman et al., 2010; Gordon et al., 2017). Molecular cross sections in the UV region, used to calculate photolysis rates (see Section 3.2), were obtained from the absorption cross sections compendium of Ranjan and Sasselov (2017) and from the work of Chen et al. (1991) via the MPI-Mainz Spectral Atlas (KellerRudek et al., 2013).

\subsection{Photochemical modeling}

In this subsection, we provide a brief description of the photochemical model used to calculate the concentration of phosphine as a function of altitude for a range of $\mathrm{PH}_{3}$ surface fluxes. We also describe the atmospheric and stellar scenarios considered in our photochemical model. We found that it is necessary to use a photochemical model instead of the approximation of fixed radical profiles (Seager et al., 2013b) because of the intense reactivity of $\mathrm{PH}_{3}$, which can drastically alter the radical profiles of an atmosphere. In particular, at high $\mathrm{PH}_{3}$ fluxes, the radical concentrations are suppressed due to reactions with $\mathrm{PH}_{3}$, meaning that $\mathrm{PH}_{3}$ can build up to much higher concentrations than a fixed radical profile approximation would predict.

3.2.1. Photochemical model. We adapt the photochemical model of $\mathrm{Hu}$ et al. (2012) to calculate atmospheric composition for different planetary scenarios. The model is detailed in the work of Hu et al. (2012); in brief, the code calculates the steady-state chemical composition of an exoplanetary atmosphere by solving the one-dimensional chemical transport equation. The model treats up to 800 chemical reactions, photochemical processes (i.e., UV photolysis of molecules), dry and wet deposition, surface emission, thermal escape of $\mathrm{H}$ and $\mathrm{H}_{2}$, and formation and deposition of elemental sulfur and sulfuric acid aerosols. The model is designed to have the flexibility of simulating both oxidized and reduced conditions. UV and visible radiation in the atmosphere is computed by the deltaEddington two-stream method. The code has been validated by reproducing the atmospheres of modern Earth and Mars. The code and extensive application examples are described in several studies (Hu et al., 2012; Hu et al., 2013; Seager et al., 2013a, 2013b; Hu and Seager, 2014). In calculating convergence, we required that the chemical variation timescale of significant species $\left(>1 \mathrm{~cm}^{-3}\right)$ to be at least $10^{19} \mathrm{~s}$, that is, longer than the age of the universe.

We added phosphine to the model of Hu et al. (2012). We considered surficial production as the only source of $\mathrm{PH}_{3}$, and rainout, photolysis, and reactions with the main radical species $\mathrm{O}, \mathrm{H}$, and $\mathrm{OH}$ as the sinks (see Section 2.3). We take the Henry's Law constant for $\mathrm{PH}_{3}$ from the work of Fu et al. (2013) via Sander (2015). For photolysis, we take the $\mathrm{PH}_{3}$ UV cross sections at $295 \mathrm{~K}$ from the work of Chen et al. (1991). We follow Kaye and Strobel (1984) in taking the branching ratio of this reaction to be unity and take the quantum yield of $\mathrm{PH}_{3}$ photolysis to be $q_{\lambda}=1$ for $\lambda<230$ $\mathrm{nm}$ and $q_{\lambda}=0$ for $\lambda>230 \mathrm{~nm}$. For the reactions with $\mathrm{OH}$, 
$\mathrm{O}$, and $\mathrm{H}$, we take the rate constants from the works of Fritz et al. (1982), Nava and Stief (1989), and Arthur and Cooper (1997), as detailed in Section 2.3. We are unaware of geochemical constraints on the dry deposition velocity of $\mathrm{PH}_{3}$; we take this value to be $0 \mathrm{~cm} \mathrm{~s}^{-1}$, which could lead to an overestimation of $\mathrm{PH}_{3}$ accumulation rates (see Section 5.2 for a discussion of possible $\mathrm{PH}_{3}$ deposition). However, our approach neglects the possibility that atmospheric photochemistry may generate $\mathrm{PH}_{3}$. In particular, we do not consider the recombination reaction, $\mathrm{PH}_{2}+\mathrm{H} \rightarrow \mathrm{PH}_{3}$. This may lead to underestimating $\mathrm{PH}_{3}$ accumulation, especially in $\mathrm{H}_{2}$-dominated atmospheres where $\mathrm{H}$ abundances are high.

3.2.2. Planetary scenarios. We model the atmospheres of Earth-sized, Earth-mass planets with two bulk atmospheric compositions: a $\mathrm{H}_{2}$-dominated atmosphere and a $\mathrm{CO}_{2}$-dominated atmosphere. We focus on $\mathrm{H}_{2}$-dominated atmospheres because their low mean molecular masses make them amenable to characterization via transmission spectroscopy (Batalha et al., 2015). We focus on $\mathrm{CO}_{2-}$ dominated atmospheres as an oxidizing end-member to complement the reducing $\mathrm{H}_{2}$-dominated case, and because early Earth is thought to have had a $\mathrm{CO}_{2}$-rich atmosphere (Kasting, 1993). We only consider anoxic atmospheres because $\mathrm{O}_{2}$-rich atmospheres are likely to have large quantities of $\mathrm{OH}$ radicals, which rapidly destroy phosphine (see Section 2.3). Additionally, the aerobic metabolism of $\mathrm{O}_{2}$ dependent life is likely to be incompatible with widespread $\mathrm{PH}_{3}$ biological production (Bains et al., 2019b).

Our atmospheres correspond to the $\mathrm{H}_{2^{-}}$and $\mathrm{CO}_{2^{-}}$ dominated benchmark scenarios of $\mathrm{Hu}$ et al. (2012), with the key difference that we do not set the rainout rates of $\mathrm{H}_{2}$, $\mathrm{CO}, \mathrm{CH}_{4}, \mathrm{C}_{2} \mathrm{H}_{6}$, or $\mathrm{O}_{2}$ to zero, as $\mathrm{Hu}$ et al. (2012) did to simulate an abiotic planet. In brief, we consider planets with surface pressures of 1 bar, surface temperatures of $288 \mathrm{~K}$, and bulk dry atmospheric composition of $10 \% \mathrm{~N}_{2}$, $90 \% \mathrm{H}_{2} / \mathrm{CO}_{2}$ for the $\mathrm{H}_{2} / \mathrm{CO}_{2}$-dominated cases, respectively. The temperature profile is taken to evolve as a dry adiabat until 160 and $175 \mathrm{~K}$ for the $\mathrm{H}_{2^{-}}$and $\mathrm{CO}_{2}$ dominated cases, respectively, and isothermally thereafter.

The strength of vertical mixing is scaled from that measured in Earth's atmosphere according to the mean molecular mass. The $\mathrm{H}_{2} \mathrm{O}$ concentration at the bottom of the atmosphere is set to 0.01 , corresponding to $60 \%$ humidity. $\mathrm{H}_{2}, \mathrm{CO}_{2}, \mathrm{CH}_{4}, \mathrm{SO}_{2}$, and $\mathrm{H}_{2} \mathrm{~S}$ are emitted from the surface at rates corresponding to terrestrial volcanism. The mixing ratio profile of the dominant gases (gases with abundances exceeding $100 \mathrm{ppb}$ ) used for the modeling of the $\mathrm{H}_{2}$-rich atmosphere on a massive super Earth orbiting an active $\mathrm{M}$ dwarf is shown in Fig. 2 (see Appendix A for the mixing ratio profiles used to model the remaining atmospheric scenarios). For further details, including the rationale for these parameters, see Hu et al. (2012).

Stellar irradiation is a key input for photochemical models. We considered instellation corresponding to the Sun (our "Sun-like" case) (Hu et al., 2012) and from the M dwarf GJ1214 (our "active M dwarf" case) (Seager et al., 2013b). The semimajor axes of the planets for the $\mathrm{H}_{2-}$ and $\mathrm{CO}_{2}$-dominated cases are taken to be 1.6 $\mathrm{AU}$ and $1.3 \mathrm{AU}$ for the Sun-like case, and $0.042 \mathrm{AU}$ and $0.034 \mathrm{AU}$ for the "active M dwarf" case, corresponding to surface temperatures of $288 \mathrm{~K}$ at $0 \mathrm{PH}_{3}$ emission.

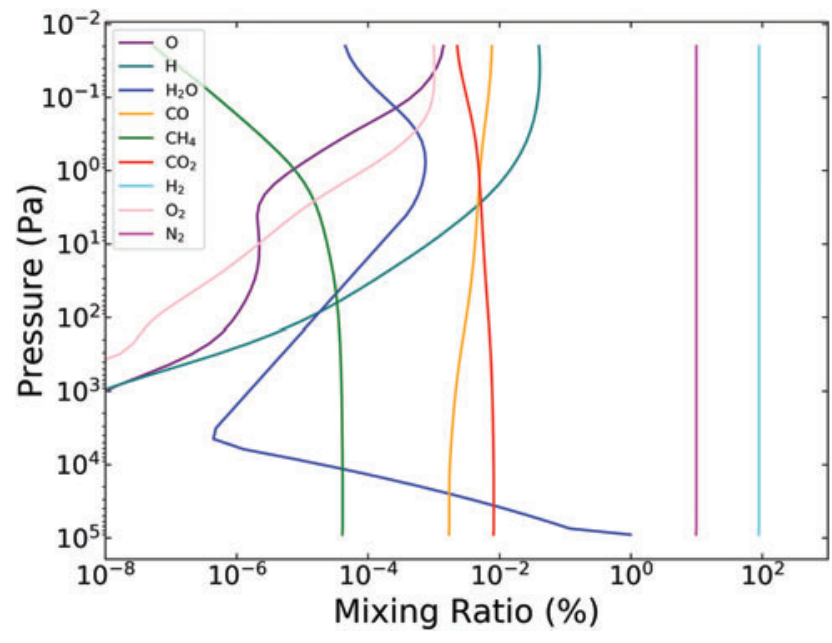

FIG. 2. Mixing ratio profile of a $\mathrm{H}_{2}$-rich atmosphere on an Earth-sized planet orbiting an active $\mathrm{M}$ dwarf. Vertical axis represents pressure in units of $\mathrm{Pa}$, and the horizontal axis shows the mixing ratio represented as a percentage of the total atmospheric layer. Figure partially adapted from the works of $\mathrm{Hu}$ et al. (2012) and Seager et al. (2013b). Color images are available online.

For a sensitivity test, we also considered a theoretical "quiet" $\mathrm{M}$ dwarf simulated by reducing the UV-flux of GJ1214 by three orders of magnitude (corresponding to approximately a factor of 100 less UV radiation than the least active M dwarf known GJ581) (France et al., 2016). See Section 4.1.4 for a discussion of the sensitivity of our results to changes in surface temperature and to low UV irradiation levels.

\subsection{Atmospheric spectral simulations}

We use the outputs of the photochemical models described above to model observational spectra projected to massive super-Earths with $M_{p}=10 M_{E}$ and $R_{p}=1.75 R_{E}$. We focus on such large massive planets due to the following observational considerations: (1) such planets are easier to detect via radial velocities and transit observations; (2) large planets have larger thermal emission signatures; and (3) massive planets are more likely to retain $\mathrm{H}_{2}$-rich atmospheres, which are much easier to characterize in transmission because of their larger scale heights than other atmosphere types.

3.3.1. Transmission and emission spectral calculations. Transmission and thermal emission spectra were simulated with the program SEAS (Simulated Exoplanet Atmosphere Spectra). The projection from Earth-sized to super-Earths is performed by using equivalent techniques to those in the work of Hu et al. (2012); SEAS takes as input a list of molecular mixing ratios as a function of pressure, which, to first order, are invariant to changes in the surface gravity.

The transmission spectrum code calculates the optical depth along the limb path and sums up chords, assuming that the planet atmosphere is homogeneous. The SEAS transmission spectrum code is similar in structure to that described in two studies (Miller-Ricci et al., 2009; Kempton 
et al., 2017), with the main difference being that SEAS can accept variable mixing ratio inputs, important for superEarths whose atmospheres are severely impacted by photochemistry. The temperature-pressure profile, including limits and resolution, is specified by the user. Molecular line lists are taken from HITRAN 2016 and ExoMol databases (Gordon et al., 2017; Tennyson et al., 2016), with cross sections calculated with HAPI (Kochanov et al., 2016) and ExoCross (Yurchenko et al., 2018), respectively. The molecular species are chosen by the user, and all molecules in the HITRAN and ExoMol databases are user-selectable options.

The thermal emission code integrates a blackbody exponentially attenuated by the optical depth without scattering (Seager, 2010). The code uses the same input temperaturepressure profile and molecular cross sections as described above.

SEAS considers clouds in the emergent spectra for thermal emission by averaging cloudy and cloud-free spectra (resulting in 50\% cloud coverage). We omit clouds or hazes for the transmission spectra model; if the atmosphere is cloudy or hazy at high altitudes, the spectral features in transmission will be muted. Consequently, our calculations represent upper bounds on the magnitude of the transmission features with respect to cloud or haze effects. We discuss the impact of clouds in our modeled transmission spectra in Section 5.2.

The SEAS transmission code has been validated by comparing results with the Atmospheric Chemistry Experiment data set (Bernath et al., 2005) for transmission spectrum and with the MODTRAN spectrum (Berk et al., 1998) for thermal emission spectrum. We also compared results related to this phosphine work with transmission spectra generated by the code described in the work of $\mathrm{Hu}$ et al. (2012).

3.3.2. Detectability metric. We study the spectroscopic detectability of phosphine in $\mathrm{H}_{2}$ - and $\mathrm{CO}_{2}$-rich atmospheres, in transmission and emission observation scenarios. In transmission, we compare with the mean transit depth of the planet radius, and in emission with the blackbody curve.

We consider a $6.5 \mathrm{~m}$ space telescope, having a quantum efficiency of $25 \%$ observing with a $50 \%$ photon noise limit. We consider our $1.75 R_{\text {Earth-planet to be orbiting (1) a } 0.26 \text { - }}$ $R_{\text {sun }} \mathrm{M}$ dwarf star at $5 \mathrm{pc}$ with an effective temperature of $3000 \mathrm{~K}$; and (2) a Sun-like star. Stellar flux is the source of the noise, combining in-transit and out-of-transit flux noises. The theoretical transmission spectra are based on JWST and its NIRSpec and MIRI instruments (Bagnasco et al., 2007; Wright et al., 2010). To calculate theoretical thermal emission spectra, we consider a secondary eclipse scenario as observed from JWST with the MIRI instruments (both mid- and low-resolution spectrometers). We binned the data to a resolution of $R \sim 10$ to increase the significance of detection.

We investigate the detectability of phosphine in exoplanet atmospheres by adapting the detection metrics defined by Seager et al. (2013b) and Tessenyi et al. (2013). The detectability metric is a theoretical metric that uses simulated data.

We first simulate model-independent observational data for all planetary scenarios considered (e.g., using the instrumental constraints of JWST). For an analysis of the transmission spectra models, we then compare the wavelength-dependent transit depth of the planet to the "white-light" transit depth in each wave band (corresponding to the coverage of each instrument).

Phosphine is considered detectable if we can detect opacity at wavelengths corresponding to $\mathrm{PH}_{3}$ absorption features with statistically significant confidence. To establish the statistical significance of opacity detection in transmission, we assume a simulated spectrum and then assign binned values for the transit depth. We then calculate the wavelength-dependent one-sigma (1- $\sigma$ ) error bar for each binned value (i.e., standard deviation) using only stellar photon noise. The significance of the deviation is calculated with:

$$
\frac{\left|\mu_{\lambda}-\mu_{\lambda}\right|}{\sqrt{\sigma_{\lambda}^{2}+\sigma_{\lambda}^{2}}}
$$

where $\mu_{\lambda}$ is the wavelength-dependent transit depth of the simulated atmosphere, $\mu_{\lambda}$ is the mean transit depth of the white-light averaged waveband, and $\sigma$ is the uncertainty on the measurement. The uncertainties are estimated based on shot noise. We then assess the detectability of phosphine by simulating a model atmosphere with and without $\mathrm{PH}_{3}$ and comparing the deviation of each atmosphere from their associated mean. This comparison establishes whether a model atmosphere with $\mathrm{PH}_{3}$ fits the simulated observational data better than one without $\mathrm{PH}_{3}$.

In thermal emission, we use a similar detectability metric to the transmission analysis described above, with the distinction that we calculate the deviation of our modeled atmosphere spectra from its best-fit blackbody continuum (instead of the white light average used for transmission comparisons). The temperature of the blackbody is set by fitting a blackbody curve to the simulated data.

The integration time is a variable parameter in the SEAS models, but features are only considered detectable if they achieve at least a 3- $\sigma$ interval with 200 observation hours or less (considering $100 \mathrm{~h}$ in-transit and $100 \mathrm{~h}$ out-of-transit).

3.3.3. Scaling to smaller planets. We performed our spectral simulations for a massive super-Earth planet $\left(M_{p}=\right.$ $10 M_{E}$ and $R_{p}=1.75 R_{E}$ ). In this section, we consider how the prospects for atmospheric characterization scale to smaller, more Earth-sized worlds.

The amplitude of the atmospheric absorption signal in transmission is characterized by Brown (2001):

$$
\frac{\delta A}{A}=\frac{2 \pi R_{p}(k T / \mu g)}{\pi R_{*}^{2}}=\frac{2 \pi R_{p}\left(k T R_{p}^{2} / \mu G M_{p}\right)}{\pi R_{*}^{2}} \propto \frac{R_{p}^{3}}{M_{p}},
$$

where $R_{p}$ is the planet radius and $M_{p}$ is the planet mass. This implies that the transmission spectroscopy signal from a $1 R_{E}, 1 M_{E}$ planet should be twice the signal from the $1.75 R_{E}, 10 M_{E}$ planet we consider here, and the phosphine surface fluxes required to produce a detectable atmospheric signal should be half of what we model for our super-Earth scenario. $^{\S}$

${ }^{\S}$ We note that it is uncertain whether a small planet can retain a $\mathrm{H}_{2}$-dominated atmosphere over geological time in the face of atmospheric escape. 
The amplitude of the thermal emission spectral signal is characterized by:

$$
F(\lambda) \propto\left[B\left(T_{\text {cont }}, \lambda\right)-B\left(T_{\text {line }}, \lambda\right)\right] R_{p}^{2},
$$

where $\lambda$ is the wavelength, $B$ is the blackbody function, and $T_{\text {cont }}$ and $T_{\text {atm }}$ are, respectively, the brightness temperature in and out of the spectral line under consideration. The above equation implies that the thermal emission signal from a $1 R_{E}, 1 M_{E}$ planet should be a third of the signal from the $1.75 R_{E}, 10 M_{E}$ planet we consider here, and the $\mathrm{PH}_{3}$ surface fluxes required to produce a detectable atmospheric signal should be three times larger than what we model for our super-Earth scenario.

We conclude that spectrally characterizing Earth-sized planets is comparable in difficulty to characterizing super Earth planets, but that characterizing the atmospheres of smaller worlds is somewhat easier in transmission and somewhat harder in emission. These differences do not affect our main conclusions.

\section{Results}

We find that phosphine as a detectable biosignature gas has three encouraging properties: (1) $\mathrm{PH}_{3}$ can accumulate to detectable levels in an exoplanet atmosphere, provided it has a high production rate at the planet's surface (Section 4.1); (2) $\mathrm{PH}_{3}$ has unique spectral features, namely strong bands around $2.7-3.6,4.0-4.8$, and $7.8-11.5 \mu \mathrm{m}$, which allow it to be distinguishable from other dominant atmosphere molecules (Section 4.2); and (3) based on the abundances and surface fluxes needed to produce detectable levels of $\mathrm{PH}_{3}$, it has no known false positives provided that the planet's surface temperature is below $800 \mathrm{~K}$ (Section 4.3). In addition to the above, our results show that, at surface fluxes near the minimum flux necessary to allow for $\mathrm{PH}_{3}$ detection, a "runaway" effect for $\mathrm{PH}_{3}$ occurs that drastically alters the atmosphere (Section 5.1).

We present results for the phosphine detection in $\mathrm{H}_{2}$ - and $\mathrm{CO}_{2}$-rich atmospheres, for planets orbiting Sun-like stars and active $\mathrm{M}$ dwarf stars.

\subsection{Phosphine detection in exoplanet atmospheres}

We performed a series of simulations and calculations to explore the prospects for detecting phosphine in an exoplanet atmosphere using transmission and thermal emission spectroscopy. We consider $\mathrm{H}_{2-}$ and $\mathrm{CO}_{2}$-dominated atmospheres, and stellar irradiation environments corresponding to the modern Sun ("Sun-like") and GJ1214 ("active M dwarf"); see Seager et al. (2013b) for details.

In this section, we first present a set of simulated spectra, both in transmission (Section 4.1.1) and in emission (Section 4.1.2), for atmospheric scenarios with and without phosphine. Here, we predict the minimum abundances required for $\mathrm{PH}_{3}$ to be detectable in each atmosphere considered. We calculate the $\mathrm{PH}_{3}$ surface production rates needed for $\mathrm{PH}_{3}$ to achieve the abundances required for detection in Section 4.1.3, using our photochemical model to simulate the equilibrium distribution of atmospheric gases throughout each atmosphere. Finally, we assess the sensitivity of our results to a variable surface temperature and a host star with low levels of radiation (Section 4.1.4).
4.1.1. Amount of phosphine required for detection via transmission spectroscopy. We find that phosphine is detectable in anoxic atmospheres only if it is able to accumulate to the order of ppb to $100 \mathrm{~s}$ of $\mathrm{ppm}$, for $\mathrm{H}_{2-}$ and $\mathrm{CO}_{2-}$ rich atmospheres, respectively. For comparison, $\mathrm{PH}_{3}$ is present at the ppt to ppb level on modern Earth. We estimate the photochemical plausibility of $\mathrm{PH}_{3}$ accumulating to such large abundances in Section 4.1.3.

Unfortunately, even with high concentrations of phosphine in the atmosphere, many tens of hours of JWST time are needed to detect it. The atmosphere mixing ratios, the surface production rates required, and the number of observation hours needed for different planet and host star scenarios are provided in Table 1.

Planets with $\mathrm{H}_{2}$-rich atmospheres orbiting active $\mathrm{M}$ dwarfs require the smallest phosphine abundances for its detection (10s to 100s of ppb; Fig. 3), which can be expected due to their lower mean radical concentrations compared with an oxidized atmosphere (Seager et al., 2013b). $\mathrm{H}_{2}$-rich atmospheres also have transmission spectra that are easier to detect than planets with higher mean molecular-weight atmospheres (e.g., $\mathrm{CO}_{2}$ ) because of their larger scale height, that is, a "puffier" atmosphere.

For $\mathrm{H}_{2}$-rich atmospheres, only the strongest band of phosphine at $4.0-4.8 \mu \mathrm{m}$ can be detectable (Fig. 3). The other $\mathrm{PH}_{3}$ features are either too weak or contaminated by other atmospheric molecular species.

For $\mathrm{CO}_{2}$-rich atmospheres (Fig. 4), several spectral bands of phosphine show substantial spectral absorptions when compared with atmospheres without $\mathrm{PH}_{3}$. Nonetheless, no spectral band of $\mathrm{PH}_{3}$ can achieve a 3- $\sigma$ statistical significance even after 200 observation hours when considering a planet orbiting an active $\mathrm{M}$ dwarf.

Phosphine is very difficult to detect on planets orbiting Sun-like stars. Planets with $\mathrm{CO}_{2}$-dominated atmospheres require longer than 200 observation hours for the detection of $\mathrm{PH}_{3}$ in transmission spectra, even with the highest surface fluxes considered $\left(3 \times 10^{13} \mathrm{~cm}^{-2} \mathrm{~s}^{-1}\right)$. The detection of $\mathrm{PH}_{3}$ can only achieve a $3-\sigma$ statistical significance on planets with $\mathrm{H}_{2}$-rich atmospheres for fluxes of $10^{14} \mathrm{~cm}^{-2} \mathrm{~s}^{-1}(\mathrm{Ta}-$ ble 1); this flux is comparable to the highest recorded $\mathrm{PH}_{3}$ flux on Earth (above sewage plants) (Devai et al., 1988) and above the values for the biological production of methane, which on Earth corresponds to $1.2 \times 10^{11} \mathrm{~cm}^{-2} \mathrm{~s}^{-1}$ (Segura et al., 2005; Guzmán-Marmolejo and Segura, 2015).

The results presented above show that it is possible, but difficult, to detect phosphine in anaerobic atmospheres if it is present as a trace gas. However, if $\mathrm{PH}_{3}$ production rates increase sufficiently, they outpace the ability of stellar NUV photons to destroy $\mathrm{PH}_{3}$, whether via direct photolysis or via generation of radical species. $\mathrm{PH}_{3}$ may then become a significant component of the atmosphere (e.g., 100s to 1000s of ppm), and its detectability increases dramatically. The $\mathrm{PH}_{3}$ surface fluxes required to reach this runaway phase $\left(\sim 10^{12}\right.$ $\left.\mathrm{cm}^{-2} \mathrm{~s}^{-1}\right)$ are not significantly higher than those required for detection $\left(\sim 10^{10-11} \mathrm{~cm}^{-2} \mathrm{~s}^{-1}\right)$. For example, with surface production rates only nine times larger than those that produce the atmospheric spectrum shown in Fig. $3, \mathrm{PH}_{3}$ reaches the runaway threshold and can be detected with 5- $\sigma$ statistical significance after only $3 \mathrm{~h}$ of observation (Fig. 5). The plausibility of this runaway effect is discussed further in Sections 4.1.3 and 5.1. 
Table 1. Phosphine Mixing Ratios Needed for Detection in Transmission for Different Atmospheric and Stellar Scenarios, with Associated Observation and Surface Flux Requirements

\begin{tabular}{lccc}
\hline Atmospheric scenario & $\begin{array}{c}\text { Required mixing } \\
\text { ratio for detection }\end{array}$ & $\begin{array}{c}\text { Minimum observation } \\
\text { hours (in-transit }+ \text { out- } \\
\text { of-transit) }\end{array}$ & $\begin{array}{c}\text { Associated confidence interval } \\
\text { for phosphine detection }(\sigma)\end{array}$ \\
\hline $\mathrm{H}_{2}$-dominated, Sun-like star & $780 \mathrm{ppm}$ & 56 & 3 \\
$\mathrm{H}_{2}$-dominated, active M dwarf (Fig. 3) & $220 \mathrm{ppb}$ & 91 & 3 \\
$\mathrm{H}_{2}$-dominated, active M dwarf & $220 \mathrm{ppb}$ & 200 & 4.4 \\
$\mathrm{H}_{2}$-dominated, active M dwarf & $5 \mathrm{ppb}$ & 200 & 2.5 \\
$\mathrm{H}_{2}$-dominated, active M dwarf & $0.28 \%$ & 3 & 5 \\
$\mathrm{CO}_{2}$-dominated, Sun-like star & $\mathrm{N} / \mathrm{A}$ & Not detectable & N/A \\
$\mathrm{CO}_{2}$-dominated, active M dwarf (Fig. 4) & $310 \mathrm{ppm}$ & 200 & 2.7 \\
$\mathrm{CO}_{2}$-dominated, active M dwarf & $7.6 \%$ & 32 & 3 \\
\hline
\end{tabular}

For planets orbiting an active $\mathrm{M}$ dwarf, phosphine requires minimum abundances of $220 \mathrm{ppb}$ and $>310 \mathrm{ppm}$ to be detectable with a $3-\sigma$ confidence interval on $\mathrm{H}_{2}$ - and $\mathrm{CO}_{2}$-rich atmospheres, respectively. Values in italics correspond to atmospheric scenarios where $\mathrm{PH}_{3}$ is at a runaway threshold (see Fig. 5 and Sections 4.1.3 and 5.1). For planets orbiting a Sun-like star, $\mathrm{PH}_{3}$ must become a major component of the atmosphere for its detection to be possible with less than 200 observation hours or, in the case of $\mathrm{H}_{2}$-rich atmospheres, with surface production rates above those found anywhere on Earth $\left(\right.$ i.e., $>10^{14} \mathrm{~cm}^{-2} \mathrm{~s}^{-1}$ ). For comparison, biological production of $\mathrm{CH}_{4}$ on Earth corresponds to $1.2 \times 10^{11} \mathrm{~cm}^{-2} \mathrm{~s}^{-1}$, of which a significant proportion is due to human activity (Houghton, 1995; Segura et al., 2005; Guzmán-Marmolejo and Segura, 2015).
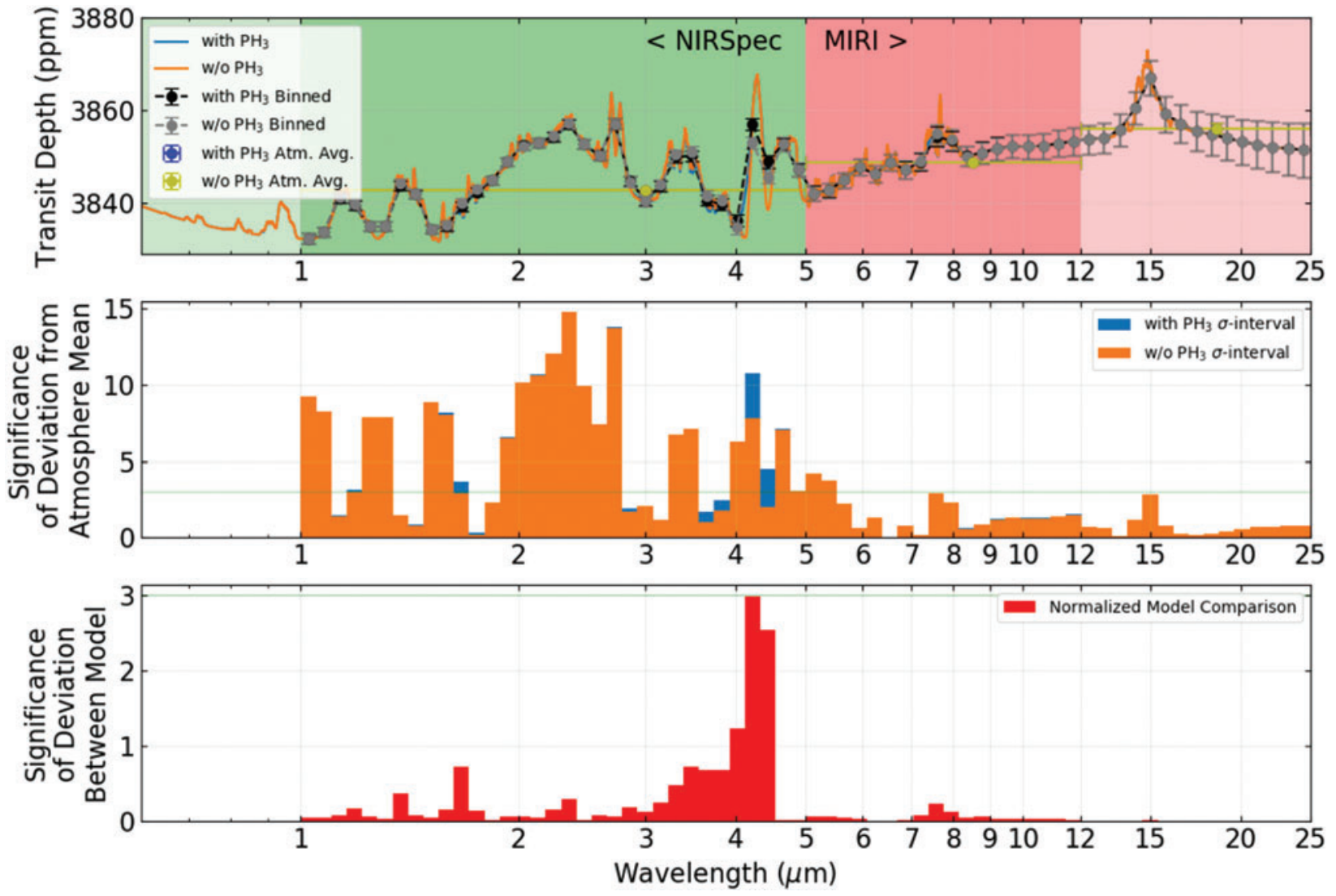

FIG. 3. Theoretical transmission spectra for a $\mathrm{H}_{2}$-rich atmosphere on a $10 M_{E}, 1.75 R_{E}$ planet with a surface temperature of $288 \mathrm{~K}$ orbiting an active $\mathrm{M}$ dwarf ( 1 bar atmosphere composed of $90 \% \mathrm{H}_{2}$ and $10 \% \mathrm{~N}_{2}$ ), after $91 \mathrm{~h}$ of observation. Top panel: vertical axis shows transit depth of the simulated atmosphere spectra in units of ppm ( $y$ axis) and horizontal axis showing wavelength in microns. The orange curve corresponds to the simulated atmosphere spectrum without $\mathrm{PH}_{3}$, and the blue curve to an atmosphere spectrum with $\mathrm{PH}_{3}$, simulated considering a $\mathrm{PH}_{3}$ concentration of 220 ppb. Blue error bars correspond to the wavelength-averaged uncertainty within the instrumental waveband; black and gray error bars correspond to the uncertainty of each wavelength bin for atmosphere models with and without $\mathrm{PH}_{3}$, respectively. Green and pink shading represent the wavelength coverage of the NIRSpec and MIRI instruments (Bagnasco et al., 2007; Wright et al., 2010). Middle panel: vertical axis shows the statistical significance of detection for two different model atmospheres, with $\mathrm{PH}_{3}$ (blue) and without $\mathrm{PH}_{3}$ (orange). Bottom panel: statistical significance of the detection of $\mathrm{PH}_{3}$ opacities at each wavelength bin. Vertical axis shows size of the statistical deviation between atmosphere models with and without $\mathrm{PH}_{3}(\mathrm{units}$ of $\sigma$-interval). In the middle and bottom panels, the horizontal green line represents the 3- $\sigma$ statistical significance threshold, and the horizontal axes show the individual wavelength bins (microns). For $\mathrm{H}_{2}$-dominated atmospheres, the 4.0$4.8 \mu \mathrm{m}$ spectral band of $\mathrm{PH}_{3}$ is the most promising feature for detection in transmission. Color images are available online. 

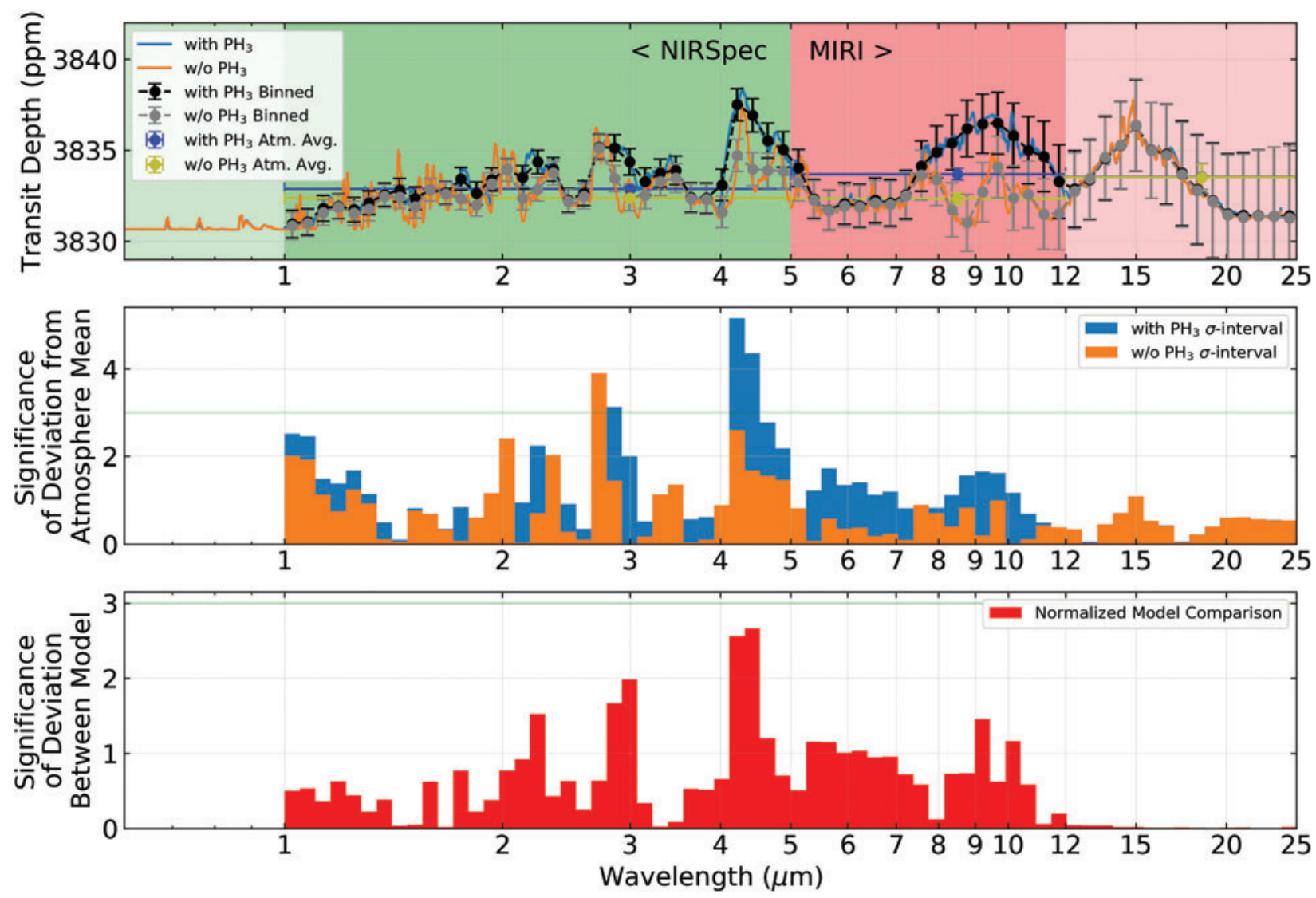

FIG. 4. Theoretical transmission spectra for a $\mathrm{CO}_{2}$-rich atmosphere on a $10 M_{E}, 1.75 R_{E}$ planet with a surface temperature of $288 \mathrm{~K}$ orbiting an active $\mathrm{M}$ dwarf ( 1 bar atmosphere composed of $90 \% \mathrm{CO}_{2}$ and $10 \% \mathrm{~N}_{2}$ ), after $200 \mathrm{~h}$ of observation. Top panel: vertical axis shows transit depth of the simulated atmosphere spectra in units of ppm ( $y$ axis), and horizontal axis showing wavelength in microns. The orange curve corresponds to the simulated atmosphere spectrum without $\mathrm{PH}_{3}$, and the blue curve to an atmosphere spectrum with $\mathrm{PH}_{3}$, simulated considering a $\mathrm{PH}_{3}$ concentration of $310 \mathrm{ppm}$. Blue error bars correspond to the wavelength-averaged uncertainty within the instrumental waveband; black and gray error bars correspond to the uncertainty of each wavelength bin for atmosphere models with and without $\mathrm{PH}_{3}$, respectively. Green and pink shading represent the wavelength coverage of the NIRSpec and MIRI instruments (Bagnasco et al., 2007; Wright et al., 2010). Middle panel: vertical axis shows the statistical significance of detection for two model atmospheres, with $\mathrm{PH}_{3}(\mathrm{blue})$ and without $\mathrm{PH}_{3}$ (orange). Bottom panel: statistical significance of the detection of $\mathrm{PH}_{3}$ opacities at each wavelength bin. Vertical axis shows size of the statistical deviation between atmosphere models with and without $\mathrm{PH}_{3}$ (units of $\sigma$-interval). In the middle and bottom panels, the horizontal green line represents the 3- $\sigma$ statistical significance threshold, and the horizontal axes show the individual wavelength bins (microns). In $\mathrm{CO}_{2}$-dominated atmospheres, several spectral features of $\mathrm{PH}_{3}$ have substantial opacities, but no feature achieves a 3- $\sigma$ statistical significance when compared with the model atmosphere without $\mathrm{PH}_{3}$. Color images are available online.

4.1.2. Amount of phosphine required for detection via emission spectroscopy. We now examine the influence of phosphine in the simulated emission spectra of $\mathrm{H}_{2}$ - and $\mathrm{CO}_{2}$-rich planets orbiting Sun-like stars and active $\mathrm{M}$ dwarfs.

Our findings on the amount of phosphine required for detection in thermal emission are similar in transmission, that is, $\mathrm{PH}_{3}$ can only be detected with many tens of hours of observation time (Table 2). We find that, in emission, $\mathrm{PH}_{3}$ is detectable in anoxic atmospheres only if it accumulates to at least abundances in the order of ppb; for comparison, $\mathrm{PH}_{3}$ is present at the ppt to ppb level on modern Earth. The photochemical plausibility of $\mathrm{PH}_{3}$ accumulating to such large abundances is presented in Section 4.1.3.

Detecting an opacity corresponding to an atmospheric spectral feature is much easier than assigning an atmospheric feature to a particular molecule. Our models show that the detection of a $\mathrm{CO}_{2}$ - or $\mathrm{H}_{2}$-rich atmosphere with high statistical significance $(>5-\sigma)$ is feasible with only a few tens of observation hours. However, the unambiguous attribution of opacity to phosphine requires much longer observation times (Table 2). As an observer, a detection can be considered as an offset to the blackbody curve, but these are only reliable if the blackbody temperature has been accurately estimated. Our detection metric uses a blackbody curve created from a best fit to the simulated observations, which biases toward low temperatures by non- $\mathrm{PH}_{3}$ absorbers. In reality the temperature of the planet may be obtained through other methods, so our results can be considered a conservative estimate for the minimum $\mathrm{PH}_{3}$ abundances required for detection.

We find the most detectable spectral region of phosphine in thermal emission is the broad band at $7.8-11.5 \mu \mathrm{m}$ (Figs. 6 and 7). In emission, planets orbiting an active $\mathrm{M}$ 

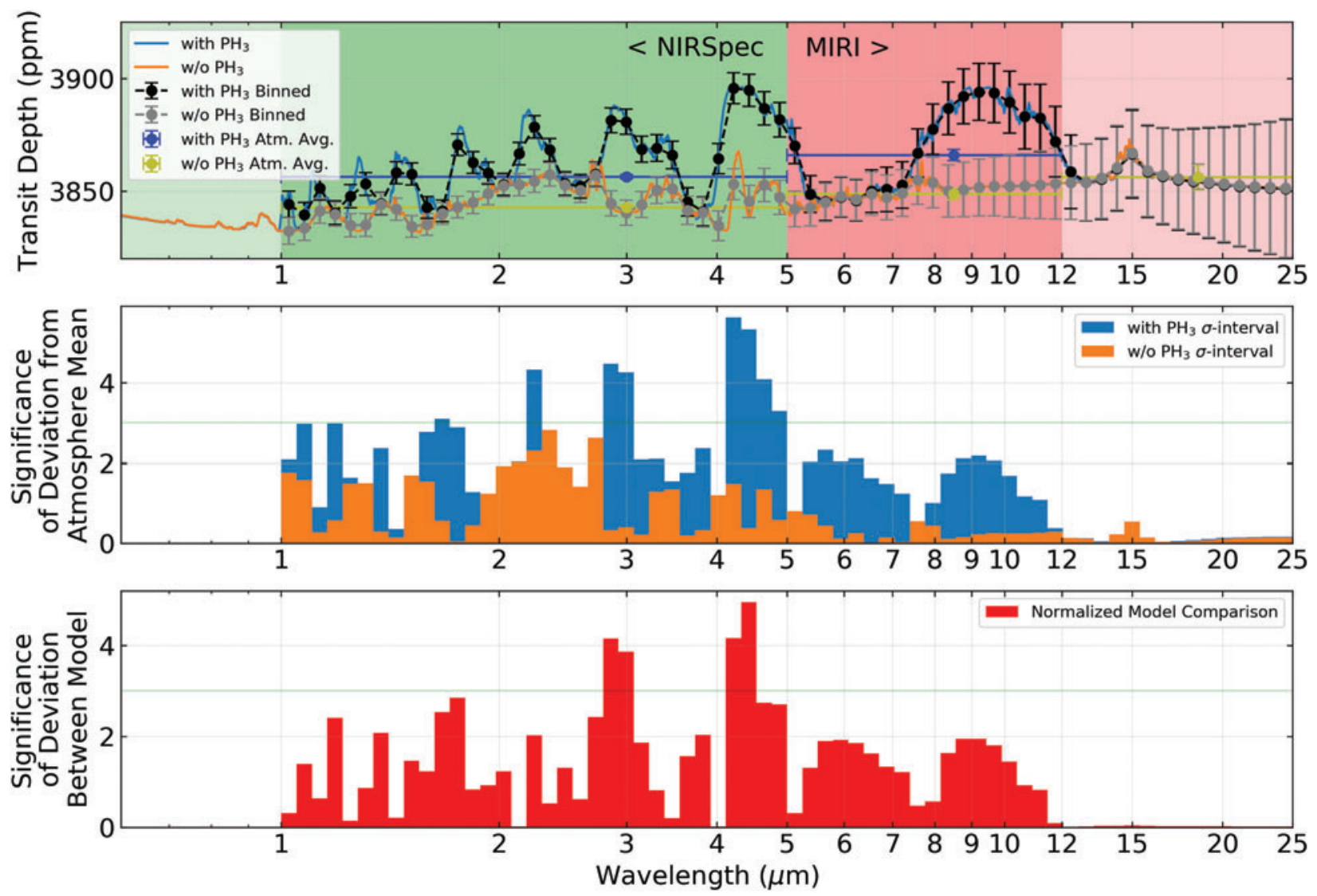

FIG. 5. Theoretical transmission spectra for a $\mathrm{H}_{2}$-rich atmosphere on a $10 M_{E}, 1.75 R_{E}$ planet with a surface temperature of $288 \mathrm{~K}$ orbiting an active $\mathrm{M}$ dwarf (1 bar atmosphere composed of $90 \% \mathrm{H}_{2}$ and $10 \% \mathrm{~N}_{2}$ ), at the threshold of the phosphine runaway phase. Top panel: vertical axis shows transit depth of the simulated atmosphere spectra in units of ppm ( $y$ axis), after $3 \mathrm{~h}$ of observation, and horizontal axis showing wavelength in microns. The orange curve corresponds to an atmosphere spectrum without $\mathrm{PH}_{3}$, and the blue curve to an atmosphere spectrum with $\mathrm{PH}_{3}$, simulated considering a $\mathrm{PH}_{3}$ concentration of $0.28 \%$. Blue error bars correspond to the wavelength-averaged uncertainty within the instrumental waveband; black and gray error bars correspond to the uncertainty of each wavelength bin for atmosphere models with and without $\mathrm{PH}_{3}$, respectively. Green and pink shading represent the wavelength coverage of the NIRSpec and MIRI instruments (Bagnasco et al., 2007; Wright et al., 2010). Middle panel: vertical axis shows the statistical significance of detection for two model atmospheres with $\mathrm{PH}_{3}$ (blue) and without $\mathrm{PH}_{3}$ (orange). Bottom panel: statistical significance of the detection of $\mathrm{PH}_{3}$ opacities at each wavelength bin. Vertical axis shows size of the statistical deviation between atmosphere models with and without $\mathrm{PH}_{3}$ (units of $\sigma$-interval). In the middle and bottom panels, the horizontal green line represents the 3- $\sigma$ statistical significance threshold, and the horizontal axes show the individual wavelength bins (microns). Once $\mathrm{PH}_{3}$ enters the runaway phase, it can be detected after a few hours of observations, through its two strong features in the 2.7-3.6 and 4$4.8 \mu \mathrm{m}$ regions. Color images are available online.

Table 2. Phosphine Mixing Ratios Needed for Detection in Emission for Different Atmospheric and Stellar Scenarios, with Associated Observation and Surface Flux Requirements

\begin{tabular}{lccc}
\hline Atmospheric scenario & $\begin{array}{c}\text { Required mixing } \\
\text { ratio for detection }\end{array}$ & $\begin{array}{c}\text { Minimum observation } \\
\text { hours (in-transit+out- } \\
\text { of-transit) }\end{array}$ & $\begin{array}{c}\text { Associated confidence } \\
\text { interval for phosphine } \\
\text { detection }(\sigma)\end{array}$ \\
\hline $\mathrm{H}_{2}$-dominated, Sun-like star & N/A & Not detectable & N/A \\
$\mathrm{H}_{2}$-dominated, active M dwarf (Fig. 6) & $220 \mathrm{ppb}$ & 131 & 3 \\
$\mathrm{H}_{2}$-dominated, active M dwarf & $4 \mathrm{ppm}$ & 52 & 3 \\
$\mathrm{CO}_{2}$-dominated, Sun-like star & N/A & Not detectable & N/A \\
$\mathrm{CO}_{2}$-dominated, active M dwarf (Fig. 7) & $15 \mathrm{ppm}$ & 150 & 3 \\
$\mathrm{CO}_{2}$-dominated, active M dwarf & $310 \mathrm{ppm}$ & 48 & 3 \\
\hline
\end{tabular}

For planets orbiting an active $\mathrm{M}$ dwarf, phosphine requires minimum abundances of $220 \mathrm{ppb}$ and $15 \mathrm{ppm}$ to be detectable on $\mathrm{H}_{2^{-}}$and $\mathrm{CO}_{2}$-rich atmospheres, respectively. For planets orbiting a Sun-like star, no scenario where $\mathrm{PH}_{3}$ is not a major component of the atmosphere could allow for its detection with less than 200 observation hours. 

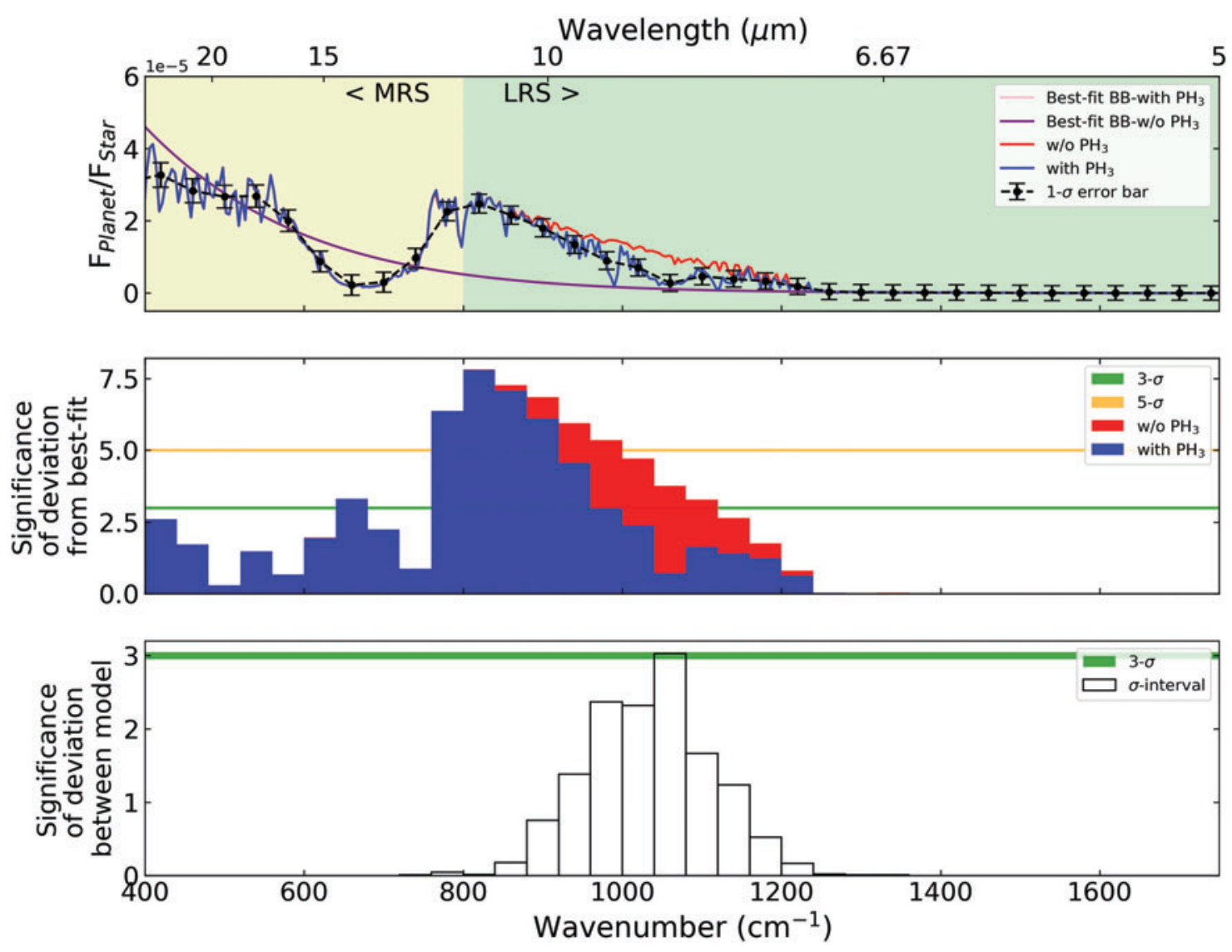

FIG. 6. Detectability of phosphine in the emission spectrum of a super Earth exoplanet $\left(10 M_{E}\right.$ and $\left.1.75 R_{E}\right)$ with a $\mathrm{H}_{2^{-}}$ rich atmosphere orbiting an active $\mathrm{M}$ dwarf, after $131 \mathrm{~h}$ of observation. Horizontal axes show wavelength in microns (top) and wave numbers in $\mathrm{cm}^{-1}$ (bottom). Top panel: vertical axes show the flux ratio between the star and the planet; pink and purple lines represent the blackbody curves fitted to the simulated observational data for atmospheres with and without $\mathrm{PH}_{3}$, respectively; blue and red curves represent a modeled atmosphere with a $\mathrm{PH}_{3}$ mixing ratio of 220 ppb and an atmosphere without $\mathrm{PH}_{3}$, respectively; black error bars represent the 1- $\sigma$ uncertainty in the observed data; MRS (yellow shading) and LRS (green shading) represent the coverage of the JWST mid- and low-resolution MIRI instruments, respectively. Middle panel: statistical significance of the detection of an atmosphere with (blue) and without (red) $\mathrm{PH}_{3}$ when compared with their best-fit blackbody curves, in units of $\sigma$-interval; the horizontal green and orange lines represent the 3- $\sigma$ and 5- $\sigma$ statistical significance threshold, respectively. Bottom panel: statistical significance of the deviation between an atmospheric model with and without $\mathrm{PH}_{3}$; the horizontal green line represents the 3- $\sigma$ statistical significance threshold. The detection of $\mathrm{PH}_{3}$ achieves a 3- $\sigma$ confidence interval through the high-frequency wing of its strong broad band at 7.8-11.5 $\mu \mathrm{m}$. JWST, James Webb Space Telescope. Color images are available online.

dwarf require the smallest $\mathrm{PH}_{3}$ abundances (100s of ppb to $100 \mathrm{~s}$ of $\mathrm{ppm}$ ) to confirm its detection, achieving a $3-\sigma$ confidence interval with a minimum of 52 and $48 \mathrm{~h}$ of observation, for $\mathrm{H}_{2-}$ and $\mathrm{CO}_{2}$-rich atmospheres, respectively.

Detection of phosphine, in emission, on planets orbiting Sun-like stars is difficult. In these scenarios, the detection of any modeled super-Earth atmosphere cannot achieve a 3- $\sigma$ confidence interval even with 200 observation hours.

We note that, at sufficiently high phosphine concentrations, our model shows that the wings of the $\mathrm{PH}_{3}$ absorption features become opaque (e.g., the strong broad band at 7.8$11 \mu \mathrm{m})$ and our emission spectra probe the isothermal stratosphere. Consequently, if $\mathrm{PH}_{3}$ concentrations are high enough, our models show that it is not possible to detect wavelengthdependent opacities due to $\mathrm{PH}_{3}$ on the basis of emission data alone. At face value, this observation implies a maximum $\mathrm{PH}_{3}$ concentration and flux, past which it is impossible to detect $\mathrm{PH}_{3}$ in emission. However, in reality this effect is an artifact of our assumption of an isothermal stratosphere. The stratosphere may have temperature variations, which would facilitate the detection of wavelength-dependent opacity variations due to $\mathrm{PH}_{3}$. A coupled climate-photochemistry model that can provide self-consistent temperature-pressure profiles is required to probe this scenario.

4.1.3. Phosphine surface fluxes required for detection. More critical than atmosphere abundances is the surface flux (i.e., the biological production rate) required for phosphine to accumulate to detectable abundance levels in anoxic atmospheres. This quantity plays a key role in determining the efficacy of $\mathrm{PH}_{3}$ as a biosignature: if the presence of detectable levels of $\mathrm{PH}_{3}$ in an atmosphere requires surface fluxes of $\mathrm{PH}_{3}$ that are higher than those which a biosphere could plausible generate, then it is disfavored as a biosignature gas; if, however, $\mathrm{PH}_{3}$ accumulates to detectable concentrations at fluxes within the range of 

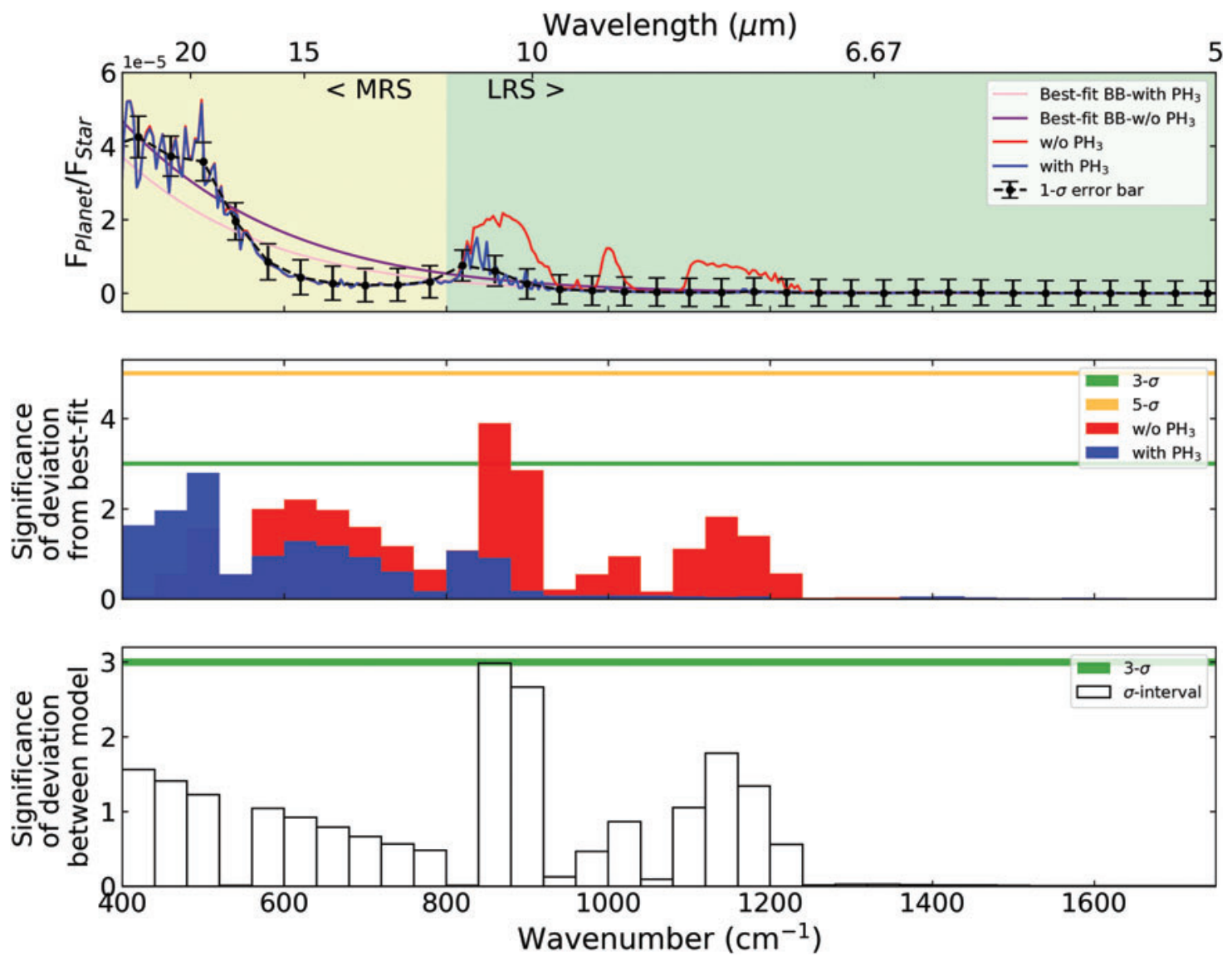

FIG. 7. Detectability of phosphine in the emission spectrum of a super Earth exoplanet $\left(10 M_{E}\right.$ and $\left.1.75 R_{E}\right)$ with a $\mathrm{CO}_{2}$ rich atmosphere orbiting an active $\mathrm{M}$ dwarf, after $48 \mathrm{~h}$ of observation. Horizontal axes show wavelength in microns (top) and wave numbers in $\mathrm{cm}^{-1}$ (bottom). Top panel: vertical axes show the flux ratio between the star and the planet; pink and purple lines represent the blackbody curves fitted to the simulated observational data for an atmosphere with and without $\mathrm{PH}_{3}$, respectively; blue and red curves represent a modeled atmosphere with a $\mathrm{PH}_{3}$ mixing ratio of $310 \mathrm{ppm}$ and an atmosphere without $\mathrm{PH}_{3}$, respectively; black error bars represent the $1-\sigma$ uncertainty in the observed data; MRS (yellow shading) and LRS (green shading) represent the coverage of the JWST mid- and low-resolution MIRI instruments, respectively. Middle panel: statistical significance of the detection of an atmosphere with (blue) and without (red) $\mathrm{PH}_{3} \mathrm{when}$ compared with their best-fit blackbody curves, in units of $\sigma$-interval; the horizontal green and orange lines represent the 3- $\sigma$ and 5- $\sigma$ statistical significance threshold, respectively. Bottom panel: statistical significance of the deviation between an atmospheric model with and without $\mathrm{PH}_{3}$; the horizontal green line represents the $3-\sigma$ statistical significance threshold. The detection of $\mathrm{PH}_{3}$ achieves a 3- $\sigma$ confidence interval through its strong broad band at 7.8-11.5 $\mu \mathrm{m}$. Color images are available online.

plausible biological productivity, it is favored as a biosignature gas.

As phosphine moves up the atmosphere, its destruction rate and consequent mixing ratio change, due to the varying levels of radical concentrations and radiation at different altitudes. The dominant $\mathrm{PH}_{3}$ reaction in $\mathrm{H}_{2}$-dominated atmospheres is $\mathrm{PH}_{3}+\mathrm{H}$. The dominant reaction in $\mathrm{CO}_{2}-$ dominated atmospheres is $\mathrm{PH}_{3}+\mathrm{O}$. However, in high- $\mathrm{PH}_{3}$ atmospheres, $\mathrm{H}$ produced from $\mathrm{PH}_{3}$ photolysis becomes an increasingly important sink for $\mathrm{PH}_{3}$, even in $\mathrm{CO}_{2}$-dominated atmospheres. $\mathrm{PH}_{3}$ is unlikely to dissolve into water and condense into aerosols (as ammonia, hydrogen sulfide, and methanethiol are) (Glindemann et al., 2003), meaning rainout is not expected to be a sink.

We use our photochemical model to estimate the minimum surface production flux, $\mathrm{P}_{\mathrm{PH} 3}$, for the detectability of phosphine in transmission and emission for a range of planetary scenarios (Table 3). We find that, for planets orbiting active $\mathrm{M}$ dwarfs, $\mathrm{PH}_{3}$ can build to concentrations detectable by transmission and emission spectroscopy if produced at the surface with rates of the order of $10^{11} \mathrm{~cm}^{-2} \mathrm{~s}^{-1}$. We note that $\mathrm{PH}_{3}$ requires similar surface flux rates in $\mathrm{H}_{2-}$ and $\mathrm{CO}_{2^{-}}$ dominant atmospheres to reach detectable abundance levels, even though those correspond to much lower $\mathrm{PH}_{3}$ concentration requirements in $\mathrm{H}_{2}$-rich atmospheres than in $\mathrm{CO}_{2}$-rich atmospheres. We speculate that this occurs because UV penetrates deeper into the more transparent $\mathrm{H}_{2}$-rich atmosphere, allowing more radical accumulation and more photolysis at depth [see Fig. 4 of Hu et al. (2012)].

The phosphine surface fluxes required to generate the detectable levels of $\mathrm{PH}_{3}$ are large when compared with global $\mathrm{PH}_{3}$ emissions on Earth but are comparable to the production rates of other major biosignature gases. For comparison, biological $\mathrm{CH}_{4}$ and isoprene production on 
Table 3. Phosphine Mixing Ratios Needed FOR DETECTION IN TRANSMISSION AND EMISSION For Different Atmospheric and Stellar Scenarios, As Well As Associated Surface Flux Requirements $\left(\mathrm{P}_{\mathrm{PH}_{3}}\left[\mathrm{CM}^{-2} \mathrm{~S}^{-1}\right]\right)$

\begin{tabular}{|c|c|c|}
\hline Atmospheric scenario & $\begin{array}{c}\text { Required mixing } \\
\text { ratio } \\
\text { for detection } \\
\text { (in transmission } \\
\text { and emission) }\end{array}$ & $\left(\begin{array}{c}P_{P_{H_{3}}} \\
\left(\mathrm{~cm}^{-2} \mathrm{~s}^{-1}\right)\end{array}\right.$ \\
\hline $\begin{array}{l}\mathrm{H}_{2} \text {-rich planet, } \\
\text { Sun-like star }\end{array}$ & $\begin{array}{c}780 \mathrm{ppm} \\
\text { (transmission) }\end{array}$ & $1 \times 10^{14}$ \\
\hline $\begin{array}{l}\mathrm{H}_{2} \text {-rich planet, active } \\
\text { M dwarf }\end{array}$ & $\begin{array}{c}5 \text { ppb } \\
\text { (transmission) }\end{array}$ & $1 \times 10^{10}$ \\
\hline $\begin{array}{l}\mathrm{H}_{2} \text {-rich planet, active } \\
\quad \mathrm{M} \text { dwarf }\end{array}$ & $\begin{array}{l}220 \mathrm{ppb} \\
\text { (emission) }\end{array}$ & $1 \times 10^{11}$ \\
\hline $\begin{array}{l}\mathrm{H}_{2} \text {-rich planet, active } \\
\mathrm{M} \text { dwarf } \\
\left(\mathrm{PH}_{3} \text { runaway) }\right.\end{array}$ & $\begin{array}{c}0.28 \% \\
\text { (transmission) }\end{array}$ & $9 \times 10^{11}$ \\
\hline $\begin{array}{l}\mathrm{CO}_{2} \text {-rich planet, active } \\
\mathrm{M} \text { dwarf }\end{array}$ & $\begin{array}{c}310 \text { ppm } \\
\text { (transmission) }\end{array}$ & $3 \times 10^{11}$ \\
\hline $\begin{array}{l}\mathrm{CO}_{2} \text {-rich planet, active } \\
\mathrm{M} \text { dwarf }\end{array}$ & $\begin{array}{c}15 \text { ppm } \\
\text { (emission) }\end{array}$ & $1 \times 10^{11}$ \\
\hline $\begin{array}{l}\mathrm{CO}_{2} \text {-rich planet, active } \\
\mathrm{M} \text { dwarf } \\
\left(\mathrm{PH}_{3} \text { runaway }\right)\end{array}$ & $\begin{array}{c}7.6 \% \\
\text { (transmission) }\end{array}$ & $1 \times 10^{12}$ \\
\hline
\end{tabular}

The values in bold represent surface fluxes and associated atmospheric abundances where phosphine would be able to approach detection but would require longer than 200 hours of observation (which is longer than our allowed limit for detectability). Values in italics correspond to atmospheric scenarios where $\mathrm{PH}_{3}$ is at a runaway threshold (see Fig. 5 and Sections 4.1.3 and 5.1). For comparison, the maximum recorded surface flux of $\mathrm{PH}_{3}$ on Earth is $10^{14} \mathrm{~cm}^{-2} \mathrm{~s}^{-1}$ (above sewage plants, Devai et al., 1988), and the biological production of $\mathrm{CH}_{4}$ on Earth corresponds to $1.2 \times 10^{11} \mathrm{~cm}^{-2} \mathrm{~s}^{-1}$ (Houghton 1995; Segura et al., 2005; Guzmán-Marmolejo and Segura, 2015).

Earth are of the order of $10^{11} \mathrm{~cm}^{-2} \mathrm{~s}^{-1}$ (Guenther et al., 2006), where a significant proportion of modern terrestrial $\mathrm{CH}_{4}$ production is anthropogenic (Houghton, 1995; Segura et al., 2005; Guzmán-Marmolejo and Segura, 2015). As a further comparison, the highest recorded surface flux of $\mathrm{PH}_{3}$ on Earth is above sewage plants, where $\mathrm{PH}_{3}$ production reaches $10^{14} \mathrm{~cm}^{-2} \mathrm{~s}^{-1}$ (Devai et al., 1988).

One of our most interesting findings is the existence of a critical phosphine surface production flux, past which $\mathrm{PH}_{3}$ accumulation is efficient and the atmosphere transitions to a $\mathrm{PH}_{3}$-rich state. We term this critical flux the "tipping point." This effect appears analogous to the "CO runaway" effect identified for early Earth (Kasting et al., 1983, 1984, 2014; Zahnle, 1986; Kasting, 2014). Past the tipping point, $\mathrm{PH}_{3}$ production outpaces the ability of stellar NUV photons to destroy $\mathrm{PH}_{3}$, whether via direct photolysis or via generation of radical species. In this runaway phase, $\mathrm{PH}_{3}$ can accumulate to percent levels and pervade the atmosphere. In this case, our models show that $\mathrm{PH}_{3}$ can be detected with observation times reaching under $10 \mathrm{~h}$ (Fig. 5). The plausibility of such a $\mathrm{PH}_{3}$ runaway effect is discussed in Section 5.1.

4.1.4. Sensitivity analysis to temperature and radiation levels. Our approach prescribes a temperature-pressure profile and considers only two possible stellar scenarios (Sun-like stars and active M dwarfs). We conducted sensi- tivity analyses to assess the dependence of our results on these assumptions.

4.1.4.1. Sensitivity analysis to temperature. In our study, we assumed surface temperatures of $288 \mathrm{~K}$; in reality, worlds with a broad range of temperatures may be habitable. Temperature may affect phosphine concentrations through varying reaction rates of $\mathrm{PH}_{3}$ with radicals and through changing the concentration of $\mathrm{H}_{2} \mathrm{O}$ in the atmosphere, from which the radical species are largely derived. To test the sensitivity of our results to surface temperature, we calculated $\mathrm{PH}_{3}$ profiles for $\mathrm{CO}_{2-}$ and $\mathrm{H}_{2}$-rich atmospheres which have detectable concentrations of $\mathrm{PH}_{3}$ at $288 \mathrm{~K}$, for new surface temperatures of 273 and $303 \mathrm{~K}$. For simplicity, in calculating the dry adiabatic evolution of the lower atmosphere, we approximated the specific heat capacities at constant pressure of $\mathrm{CO}_{2}$ and $\mathrm{H}_{2}$, by their values at $273 \mathrm{~K}$ and $303 \mathrm{~K}$ (Pierrehumbert, 2010). We adjusted the surface mixing ratio of water vapor to 0.0036 and 0.026 , corresponding to the vapor pressures at 273 and $303 \mathrm{~K}$, respectively, for the same $60 \%$ humidity assumed at $288 \mathrm{~K}$. Figures 8 and 9 present the results of these sensitivity tests in the case of a $\mathrm{H}_{2}$-dominated atmosphere orbiting an $\mathrm{M}$ dwarf star.

We find phosphine abundances to be weakly sensitive to surface temperature. For both $\mathrm{CO}_{2-}$ and $\mathrm{H}_{2}$-dominated atmospheric scenarios, the total $\mathrm{PH}_{3}$ column varies by a factor of $\leq 3$ relative to the value at $288 \mathrm{~K}$ across $273-303 \mathrm{~K}$, with the strongest variation occurring in $\mathrm{H}_{2}$-dominated atmospheres. Such variations, while potentially significant for retrievals, do not affect our order-of-magnitude conclusions regarding the detectability of $\mathrm{PH}_{3}$.

We attribute this relatively modest variation of phosphine column with temperature to the comparatively small variation of both the $\mathrm{PH}_{3}$ radical reaction rates and the total water vapor column across this temperature range. Other atmospheric constituents, such as methane, show a much greater sensitivity to lower temperatures than $\mathrm{PH}_{3}$. We are unsure why this is the case. One possibility is that $\mathrm{PH}_{3}$ reaction rates are less sensitive to temperature changes than other atmospheric constituents (e.g., from 288 to $303 \mathrm{~K}$, the rate constant for $\mathrm{H}+\mathrm{CH}_{4}$ increases by a factor of 2.3 , whereas the rate constant for $\mathrm{H}+\mathrm{PH}_{3}$ increases by a factor of 1.16). Another possible explanation for $\mathrm{CH}_{4}$ having a greater sensitivity to temperature than $\mathrm{PH}_{3}$ is that $\mathrm{CH}_{4}$ is primarily removed by $\mathrm{OH}$ (and therefore most sensitive to $\mathrm{H}_{2} \mathrm{O}$ ), whereas $\mathrm{PH}_{3}$ is primarily removed by $\mathrm{O}$ and $\mathrm{H}$ (i.e., less sensitive to $\mathrm{H}_{2} \mathrm{O}$ ).

We crudely considered the potential impact of high phosphine abundances on the temperature profile of a planet. The greenhouse gas potential of $\mathrm{PH}_{3}$ is not known (Bera et al., 2009), but it is plausible that a significant accumulation of phosphine on an atmosphere would contribute to an increase in the global temperature since $\mathrm{PH}_{3}$ is a strong infrared absorber.

To first order, the change in surface temperature due to phosphine can be estimated by calculating the surface temperature required to produce enough outgoing radiation to balance the arriving stellar radiation (Pierrehumbert, 2010). We executed this procedure for an atmosphere with and without $\mathrm{PH}_{3}$. We estimate that, if $\mathrm{PH}_{3}$ accumulates to the abundances required for its detection (see Section 4.1.3), $\mathrm{PH}_{3}$ can lead to an increase of surface temperature between 10 

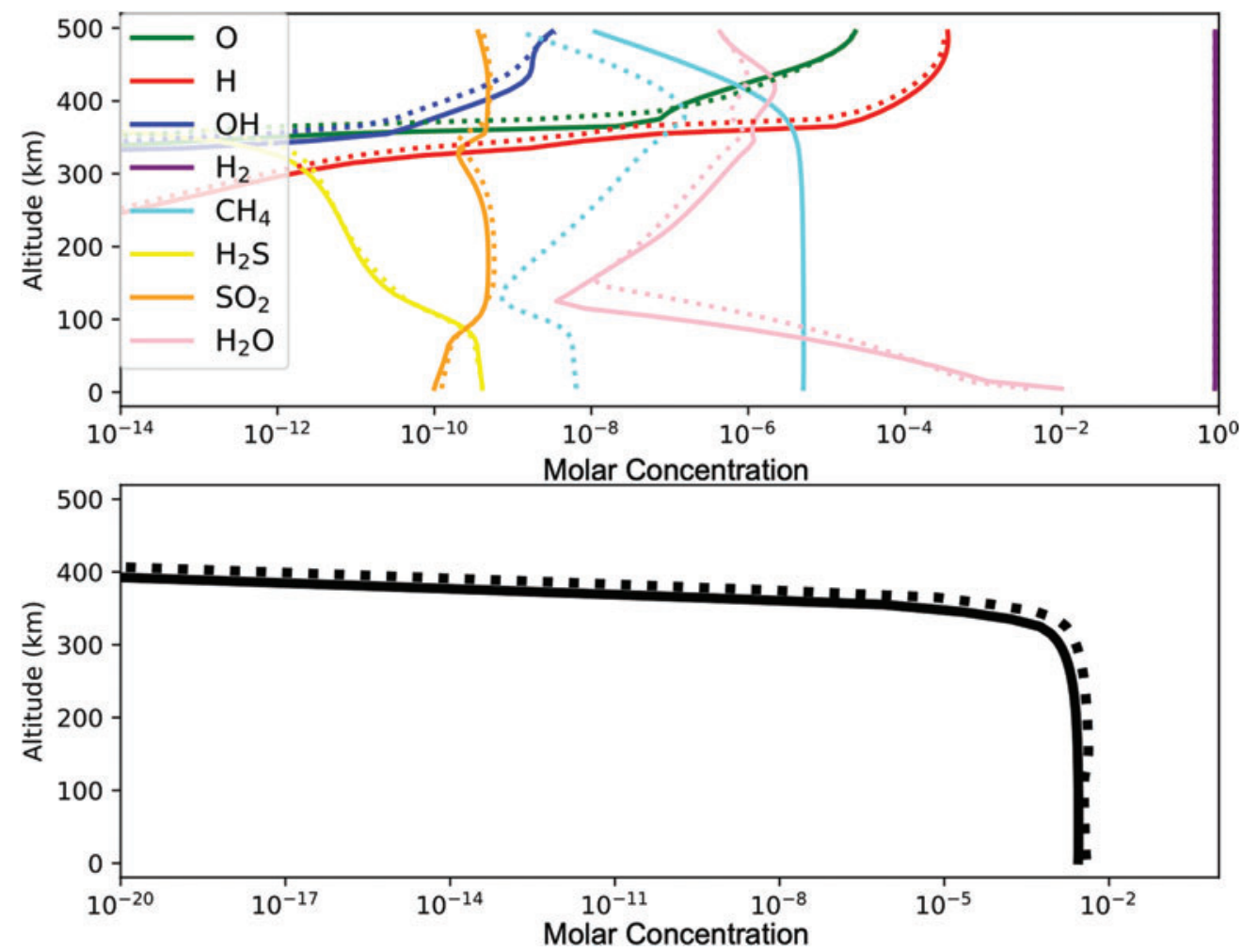

FIG. 8. Distribution of abundances of atmospheric constituents (top panel) and phosphine (bottom panel) throughout the atmosphere of a $\mathrm{H}_{2}$-rich planet orbiting an active $\mathrm{M}$ dwarf. Vertical axes represent altitude in units of $\mathrm{km}$, and horizontal axes represent molar concentration. Solid lines and dotted lines correspond to mixing ratios with surface temperatures of 288 and $273 \mathrm{~K}$, respectively. When comparing low temperatures $(273 \mathrm{~K})$ to our standard $288 \mathrm{~K} \mathrm{models}$, the $\mathrm{PH}_{3} \mathrm{mixing}$ ratio remains mostly unchanged. Color images are available online.
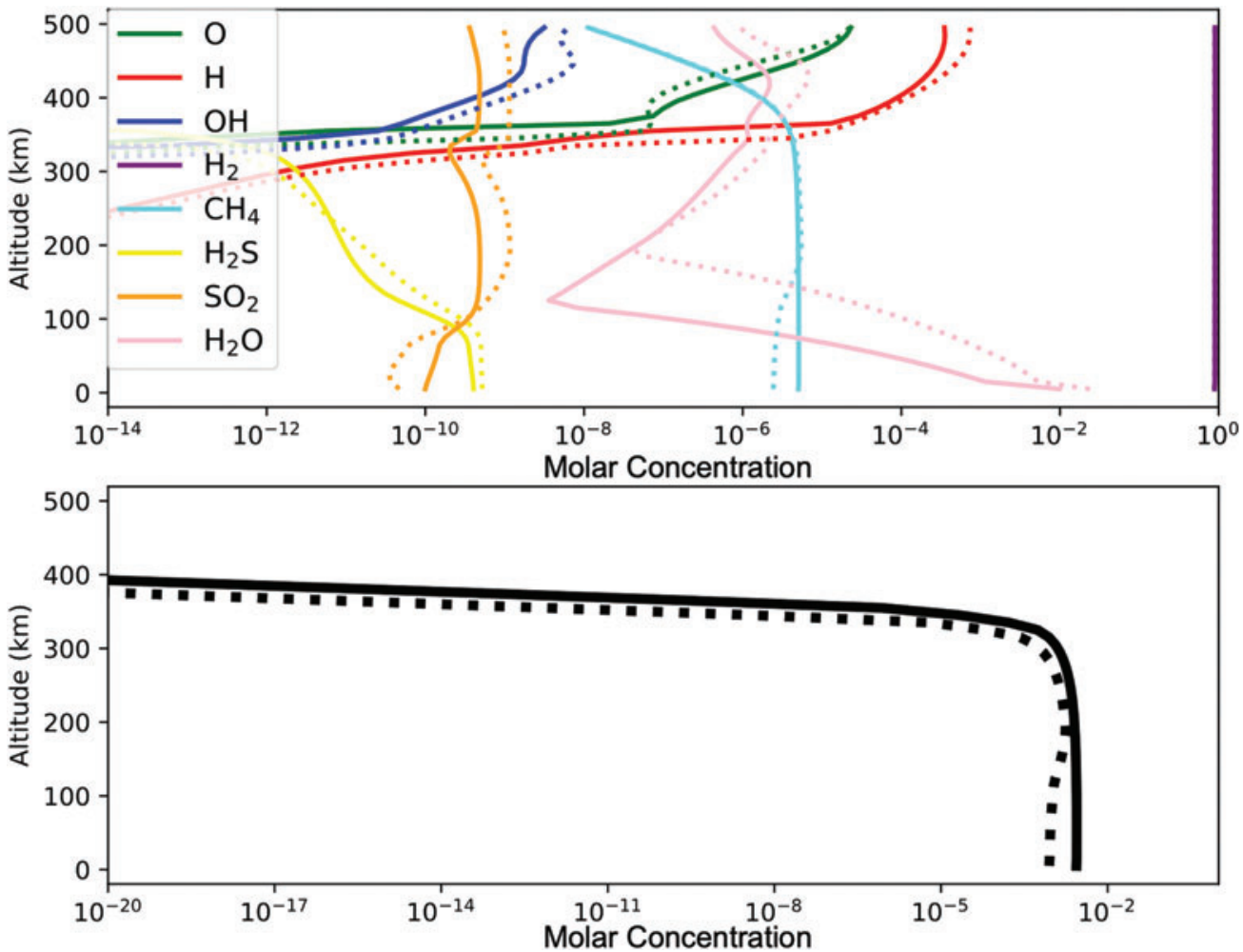

FIG. 9. Distribution of abundances of atmospheric constituents (top panel) and phosphine (bottom panel) throughout the atmosphere of a $\mathrm{H}_{2}$-rich planet orbiting an active $\mathrm{M}$ dwarf. Vertical axes represent altitude in units of $\mathrm{km}$, and horizontal axes represent molar concentration. Solid lines and dotted lines correspond to mixing ratios with surface temperatures of 288 and $303 \mathrm{~K}$, respectively. When comparing high temperatures $(303 \mathrm{~K})$ to our standard $288 \mathrm{~K} \mathrm{models}$, the $\mathrm{PH}_{3} \mathrm{mixing}$ ratio remains mostly unchanged. Color images are available online. 
and $30 \mathrm{~K}$, depending on the atmospheric scenario. Further studies on the greenhouse gas potential of $\mathrm{PH}_{3}$ are needed to fully explore the impact of its accumulation on the temperature profile of exoplanet atmospheres.

Overall, we conclude that our results are insensitive to variations in surface temperature of $\pm 15 \mathrm{~K}$.

4.1.4.2. Sensitivity to $U V$ irradiation. UV irradiation limits phosphine concentrations through direct photolysis and radical production. We considered the hypothesis that $\mathrm{PH}_{3}$ would build to higher concentrations on a planet orbiting a star with low UV output, such as a quiet M dwarf, as considered by Domagal-Goldman et al. (2011). To test this hypothesis, we simulated $\mathrm{CO}_{2}$ - and $\mathrm{H}_{2}$-rich planets orbiting a theoretical "quiet" M dwarf.

We constructed our quiet $\mathrm{M}$ dwarf model by reducing the instellation at wavelengths $<300 \mathrm{~nm}$ of our active $\mathrm{M}$ dwarf case (corresponding to GJ1214) by a factor of 1000 . This corresponds to $\sim 100$ times less UV than GJ581, the quietest M dwarf observed by the MUSCLES survey (France et al., 2016). A truly quiet M dwarf may not exist, as practically all $\mathrm{M}$ dwarfs observed to date have at least some chromospheric activity (France et al., 2013, 2016). Our quiet M dwarf case may therefore be considered a theoretical limiting case to study the effect of UV radiation on $\mathrm{PH}_{3}$ buildup, with the understanding that this limiting case may not exist in reality. We nonetheless note that this limiting case is less extreme than photosphere-only limiting cases considered in past work (Domagal-Goldman et al., 2011; Seager et al., 2013b; Rugheimer et al., 2015).

We find that, for the equivalent surface production rates, phosphine concentrations are two orders of magnitude higher on planets in the quiet $M$ dwarf cases compared with the active $M$ dwarf cases. Low UV emission favors buildup of $\mathrm{PH}_{3}$ due to lower radical concentrations and photolysis rates. Consequently, as with other proposed biosignature gases, planets orbiting quiet $\mathrm{M}$ dwarfs are the best targets for detecting biogenic $\mathrm{PH}_{3}$ (Segura et al., 2005; Domagal-Goldman et al., 2011; Seager et al., 2013b). We also find that, in planets orbiting a quiet $\mathrm{M}$ dwarf, $\mathrm{PH}_{3}$ is able to enter a runaway phase with two orders of magnitude lower surface fluxes than those required in more active stars (Section 5.1).

Our overall main finding is that, because phosphine is easily destroyed either directly by UV or indirectly by UVmediated creation of $\mathrm{H}, \mathrm{O}$, or $\mathrm{OH}$ radicals, a UV-poor environment is favorable for the detection of $\mathrm{PH}_{3}$. This result is consistent with past work (Segura et al., 2005; DomagalGoldman et al., 2011; Seager et al., 2013b). If there are no sufficiently quiet $\mathrm{M}$ dwarf stars, we speculate that a UVpoor environment can be created by a UV shield on the planet itself (Wolf and Toon, 2010). Since $\mathrm{PH}_{3}$ is readily destroyed in an $\mathrm{O}_{2}$-rich environment, an ozone UV shield is unsuitable because other oxygen-containing radicals would destroy $\mathrm{PH}_{3}$. However, elemental sulfur aerosols generated on planets with high volcanism and reducing atmospheres may provide such a UV shield ( $\mathrm{Hu}$ et al., 2013). Additionally, if $\mathrm{PH}_{3}$ fluxes are high enough, they can overwhelm the supply of destructive UV photons and build up to higher concentrations (a runaway effect). For more context and for a comparison with $\mathrm{CH}_{3} \mathrm{Cl}$, another proposed biosignature gas, see Sections 5.1 and 5.3.

\subsection{Phosphine spectral distinguishability}

Phosphine's spectral features can be easily distinguished from those of other gases expected to be main components of rocky planet atmospheres. Such gases include water vapor, methane, carbon dioxide, carbon monoxide, ammonia, and hydrogen sulfide (Fig. 10). Ammonia might be present in hydrogen-rich atmospheres (Seager et al., 2013b).

The infrared spectrum of phosphine has three major features: $2.7-3.6,4-4.8$, and $7.8-11 \mu \mathrm{m}$, corresponding to polyad $(P)$ numbers 3,2 , and 1 , respectively (Sousa-Silva et al., 2013). The $2.7-3.6 \mu \mathrm{m}$ region $(P=3)$ is dominated by a hot band and an overtone band, both associated with the symmetric bending mode of $\mathrm{PH}_{3}$, and six additional combination bands. The $4-4.8 \mu \mathrm{m}$ region $(P=2)$ is dominated by both the fundamental symmetric and asymmetric stretching bands of the $\mathrm{PH}_{3}$ molecule. The $P=2$ feature is also where several weaker, bending overtone and combination bands occur, combining with the fundamental bands to result in the strongest overall spectral feature for $\mathrm{PH}_{3}$. When compared with water, methane, ammonia, and hydrogen sulfide (but not $\mathrm{CO}_{2}$ ), the $P=2$ feature is uniquely attributable to $\mathrm{PH}_{3}$ (Fig. 10). In the $7.8-11 \mu \mathrm{m}$ region $(P=1)$, the fundamental symmetric and asymmetric bending modes, as well as hot bands, combine to produce a strong and broad absorption feature. The $P=1 \mathrm{PH}_{3}$ feature overlaps with the ammonia spectrum but is easily distinguishable from the remaining molecules in this comparison (Fig. 10).

For more detailed comparisons focusing on the 2.7-5 and 7.8-11.5 $\mu \mathrm{m}$ regions, see Appendix B.

The strongest band of phosphine, in the $4-4.8 \mu \mathrm{m}$ region, is particularly salient when comparing $\mathrm{PH}_{3}$ with all available spectra of volatile molecules (Fig. 11). However, the second strongest feature of $\mathrm{PH}_{3}$, the broad band in the 7.8$11 \mu \mathrm{m}$ region, is easily obscured by other gases as it absorbs in a heavily populated wavelength region, where many molecules have strong fundamental rovibrational modes.

It is worth noting that, of the 534 molecules for which there are available spectra, only a few dozen have been adequately measured or calculated, and consequently their spectra should be considered preliminary. Furthermore, there are thousands of volatile molecules that could contribute to an atmospheric spectra (Seager et al., 2016) for which there is no available spectral data, so further studies are required to reveal the full extent of the spectral comparison highlighted in Fig. 11 (Sousa-Silva et al., 2018, 2019).

\subsection{Phosphine false positives}

On Earth, the only significant amounts of phosphine found in the atmosphere are produced anthropogenically or biologically (Sections 2.1 and 2.2). The formation of $\mathrm{PH}_{3}$ on temperate rocky planets is thermodynamically disfavored, even in high-reducing environments, unlike the abiological production of methane or hydrogen sulfide.

In thermodynamic equilibrium, phosphorus can be conservatively expected to be found in the form of phosphine only at $T>800 \mathrm{~K}$, and at $P>0.1$ bar (Visscher et al., 2006), and only in environments rich in $\mathrm{H}_{2}$, which is why $\mathrm{PH}_{3}$ has been detected in Jupiter and Saturn, where these extreme temperatures occur (in the deep layers of the atmosphere). In $\mathrm{H}_{2}$-poor environments, much higher temperatures and pressures are required for $\mathrm{PH}_{3}$ to be thermodynamically 

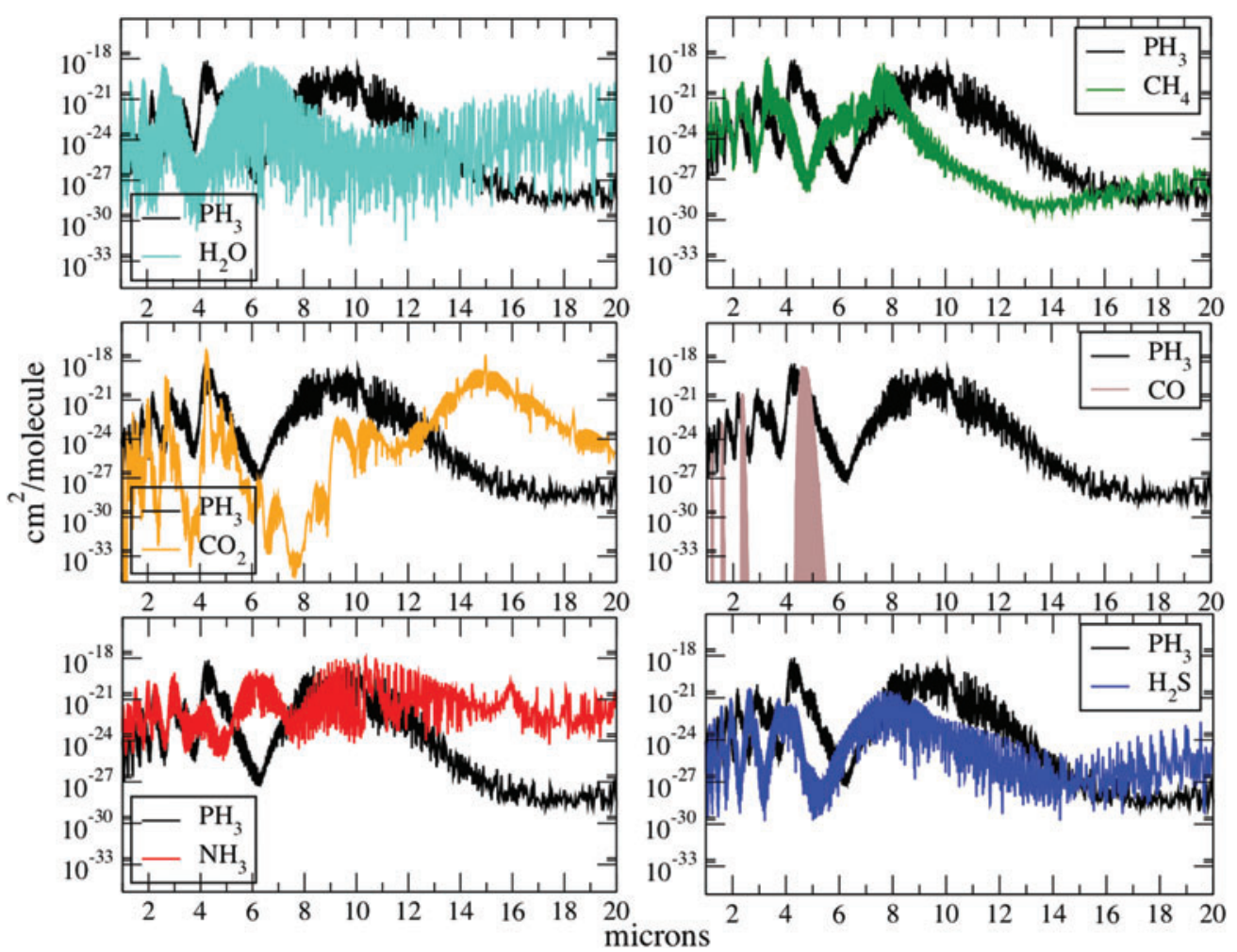

FIG. 10. Comparison of the spectral cross sections of phosphine with other molecular gases at room temperature. Intensity on $y$ axes in a log-scale with units of $\mathrm{cm}^{2} /$ molecule, and wavelength represented on the $x$ axes in microns. All cross sections are calculated at zero-pressure (i.e., Doppler-broadened lines only) using the procedure described by Hill et al. (2013). $\mathrm{PH}_{3}$, shown in black, is distinguishable from all compared molecules due to its strong bands in the 2.7-3.6, 4-4.8, and 7.8-11 $\mu \mathrm{m}$ regions. Color images are available online.

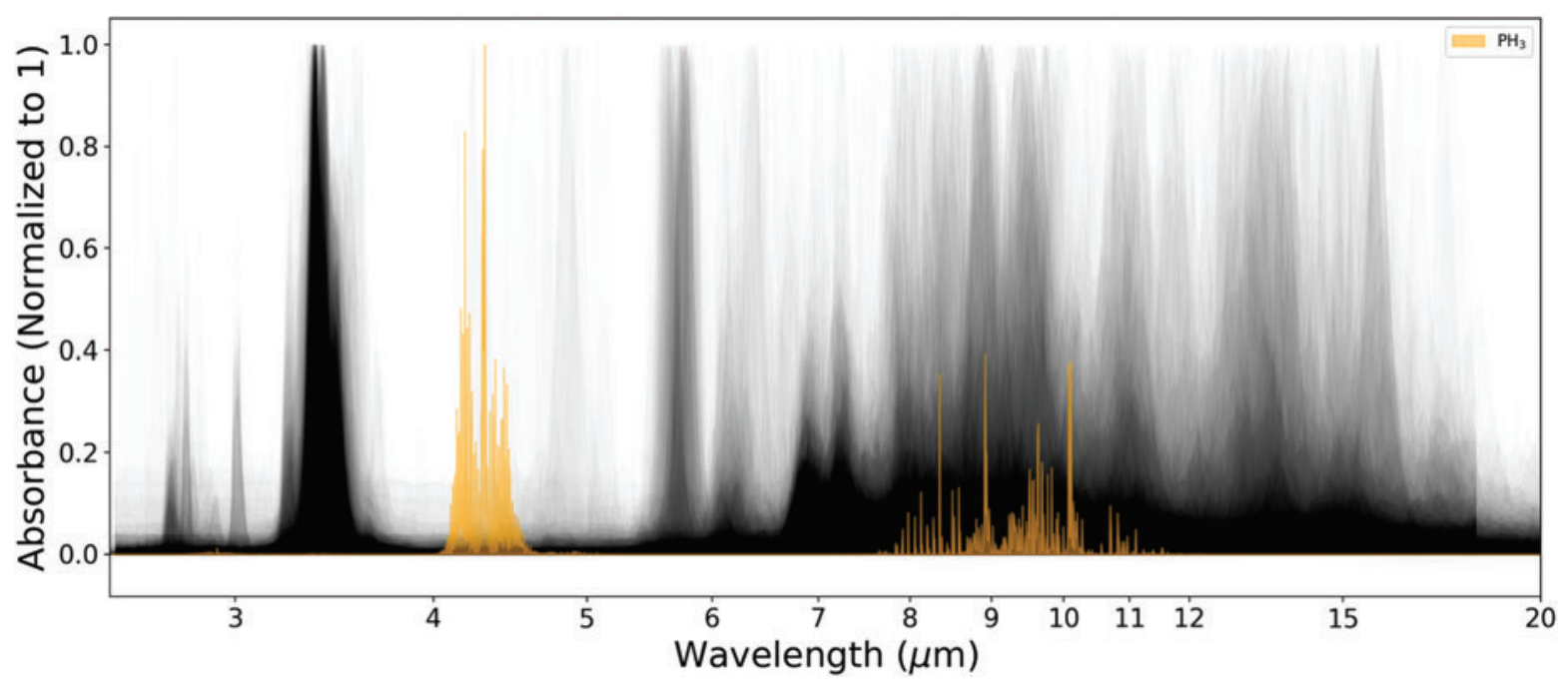

FIG. 11. Comparison of the spectral cross sections of phosphine (orange) with all the available cross sections for molecules that are volatile at room temperature (Lemmon et al., 2010). Intensity on $y$ axes in a linear scale representing absorbance (normalized to 1), and wavelength represented on the $x$ axes in microns, with the spectral range constrained to $2.5-18.5 \mu \mathrm{m}$ for fair comparison (many molecules have incomplete spectra beyond this region). Opacity for all molecules is plotted at $1 \%$ so that heavily populated regions are highlighted. All cross sections are calculated with SEAS, using molecular inputs from NIST (Linstrom and Mallard, 2001) and ExoMol (Tennyson et al., 2016). The strongest band of $\mathrm{PH}_{3}$ $(4.0-4.8 \mu \mathrm{m})$ is easily distinguishable from all other gases, but the broad band at $10 \mu \mathrm{m}$ can become obscured by other molecules. SEAS, Simulated Exoplanet Atmosphere Spectra. Color images are available online. 
favored. We also note that the critical temperature of water is $647 \mathrm{~K}$ so there are no surface conditions that favor both $\mathrm{PH}_{3}$ production and allow for the presence of liquid water. Consequently, in a temperate rocky planet, it is implausible that $\mathrm{PH}_{3}$ can be produced without biological intervention, so its detection in such an environment is a promising indication of biological activity. We summarize below the potential falsepositive scenarios for $\mathrm{PH}_{3}$ as a biosignature gas and their expected impact on the global concentrations of $\mathrm{PH}_{3}$.

Overall, nonbiological phosphine formation is not favored on temperate rocky worlds, and no abiotic pathways can produce $\mathrm{PH}_{3}$ with the production rates necessary for its detection on habitable exoplanets. We therefore conclude that, in contrast to molecules such as ammonia and methane, a detection of $\mathrm{PH}_{3}$ on a temperate exoplanet is likely to only be explained by the presence of life.

4.3.1. Formation from phosphite and phosphate. We considered the hypothesis that phosphine could be formed geochemically as a "false positive" by reduction of phosphate or phosphite to $\mathrm{PH}_{3}$. Phosphate is a dominant form of phosphorus on Earth. Phosphite is much less abundant but was detected in ground water and in mineral deposits (Han et al., 2012, 2013; Yu et al., 2015) where it is likely to be the result of biological activity (Bains et al., 2019a). Phosphite is also postulated to have been much more abundant on early anoxic Earth (Pasek, 2008; Pasek et al., 2013; Herschy et al., 2018). We calculated the Gibbs free energy of formation of $\mathrm{PH}_{3}$ from both phosphate and phosphite under geochemical source conditions at neutral $\mathrm{pH}$, for $T=273$ and $413 \mathrm{~K}$ and $\mathrm{pH}_{2}=10^{-6}$ and 1 bar. In all cases, the formation of $\mathrm{PH}_{3}$ was thermodynamically disfavored; see Appendices $\mathrm{C}$ and $\mathrm{D}$ for details. We conclude that $\mathrm{PH}_{3}$ formation from phosphate or phosphite is unlikely in the absence of a biological catalyst; for more details on the thermodynamic plausibility of the reduction of phosphites or phosphate into $\mathrm{PH}_{3}$, see Bains et al. (2019a).

Phosphite can disproportionate to phosphine at $T \gtrsim 320 \mathrm{~K}$ and acidic pH (Bains et al., 2019a), raising the possibility that "black smoker" hydrothermal systems $(T \leq 678 \mathrm{~K}$, $\mathrm{pH}=2-3$ ) (Martin et al., 2008) might generate phosphine (Appendix D). Such systems do not dominate volcanic emission on Earth, leading us to propose they would be a negligible contributor on Earth-analog worlds. However, if a world had global, hot, acidic oceans (e.g., due to very high $\mathrm{pCO}_{2}$ ), then the theoretical possibility of abiotic phosphine production exists, although likely only in the presence of high $\mathrm{H}_{2}$ concentrations, very low $\mathrm{pH}$ and within a very hot temperature band (Appendix D) (Bains et al., 2019a). Given that these oceans would be unlikely to have $\mathrm{pH}$ values below 4 (carbonic acid has a $\mathrm{pH}$ of 3.6) and $\mathrm{PH}_{3}$ formation is only favored at $\mathrm{pHs}$ closer to 2 , we consider this scenario possible but implausible.

4.3.2. Lightning. We also considered the possibility of phosphine production by lightning. Lightning discharges even in highly reducing atmospheres produce only negligible amounts of reduced phosphorus species, including $\mathrm{PH}_{3}$, and are very unlikely to provide high flux sources of $\mathrm{PH}_{3}$ globally. A few studies have examined the production of reduced phosphorus species from phosphate as a result of simulated lightning discharges in laboratory conditions
(Glindemann et al., 1999., 2004); only a very small fraction of the phosphorus was reduced to $\mathrm{PH}_{3}$ through this process, even in highly reduced atmospheric conditions. Similarly, a mineral fulgurite - a glass resulting from lightning strikeswas also proposed as a potential source of $\mathrm{PH}_{3}$ given that it could, in principle, contain reduced phosphorus species (Pasek and Block, 2009). However, these sources are rare and localized; they would have minimal impact on a global scale. We are not aware of kinetically favored reactions that would promote the conversion of the thermodynamically favored phosphate to $\mathrm{PH}_{3}$.

4.3.3. Volcanism. Phosphine is not known to be produced by volcanoes on Earth.

Calculations on the production of phosphine through volcanism on a simulated anoxic early Earth showed that only trace amounts of $\mathrm{PH}_{3}$ can be created through this avenue; the predicted maximum production rate is 102 tons per year (Holland, 1984), which corresponds to $\sim 10^{4} \mathrm{~cm}^{-2} \mathrm{~s}^{-1}$. We note that the estimation of the maximum production of $\mathrm{PH}_{3}$ through the volcanic processes reported by Holland (1984) is made under the assumption of a highly reduced planet, which provides favorable conditions for $\mathrm{PH}_{3}$ volcanic production. The volcanic production of $\mathrm{PH}_{3}$ in other planetary scenarios is even more unlikely. We estimate that the maximum production of $\mathrm{PH}_{3}$ by volcanoes in any planetary scenario, even $\mathrm{H}_{2}$-rich atmospheres, is at least seven orders of magnitude lower than the surface fluxes required for detection (see Section 4.1.3).

4.3.4. Exogenous delivery. Finally, we considered the possibility of exogenous meteoritic delivery as a source of reduced phosphorus species that could lead to the abiotic production of phosphine. Reduced phosphorus species can be found in the meteoritic mineral schreibersite (Pech et al., 2011). Schreibersite is (Fe,Ni) ${ }_{3} \mathrm{P}$, which is present in iron/ nickel meteorites (Geist et al., 2005); it is not present in stony or carbonaceous bodies. The current accretion rate of meteoritic material to Earth is of the order of 20-70 kilotons per year (Peucker-Ehrenbrink, 1996). We calculated the maximum $\mathrm{PH}_{3}$ production from these sources as follows: considering that $\sim 6 \%$ of the meteoritic material is iron/ nickel (Emiliani, 1992) and such meteorites contain an average of $0.25 \%$ phosphorus by weight (Geist et al., 2005), and working under the conservative assumption that the totality of the phosphorus content could be hydrolyzed to $\mathrm{PH}_{3}$, these meteors would deliver a maximum of $\sim 10$ tons of $\mathrm{PH}_{3}$ to Earth every year. ${ }^{* *}$ Therefore, the contribution from meteoritic sources to the global average $\mathrm{PH}_{3}$ production rates is still negligible. The above calculations are also in agreement with previous estimations of the phosphine production through meteoritic delivery, which were also found to be negligible (Holland, 1984).

\section{Discussion}

We find that phosphine is a promising marker for life if detected on a rocky exoplanet. On Earth, $\mathrm{PH}_{3}$ is naturally

\footnotetext{
${ }^{* *}$ For comparison, 10.2 million tons per year of methane are produced from ruminants alone (Moss et al. 2000).
} 
associated exclusively with anaerobic life and is not expected to have any significant false positives for life on temperate exoplanets. Our models find that, if produced at sufficiently high surface fluxes, $\mathrm{PH}_{3}$ can accumulate in planetary atmospheres to detectable abundances. Here, we discuss the photochemical impact of high abundances of $\mathrm{PH}_{3}$ in an exoplanet atmosphere (Section 5.1). We then describe the known limitations of our calculations (Section 5.2) and expand on alternative methods of detecting $\mathrm{PH}_{3}$ in exoplanet atmospheres (Section 5.3). We summarize our findings in section 5.4 .

\subsection{Phosphine "tipping point" and its impact on the atmosphere}

Our models show that, with global phosphine surface fluxes comparable to those found locally in anoxic ecosystems on Earth, $\mathrm{PH}_{3}$ can have a significant impact on planets with anoxic atmospheres. We calculate that $\mathrm{PH}_{3}$ becomes detectable on anoxic planets where it is emitted at the surface with fluxes greater than $10^{11} \mathrm{~cm}^{-2} \mathrm{~s}^{-1}$. Consequently, $\mathrm{PH}_{3}$ is accessible to remote detection only if it is a substantial product of the biosphere, emitted in quantities similar to $\mathrm{CH}_{4}$ and isoprene on Earth (Guenther et al., 2006). We note that the maximum recorded surface flux of $\mathrm{PH}_{3}$ on Earth is $10^{14} \mathrm{~cm}^{-2} \mathrm{~s}^{-1}$ (Devai et al., 1988). $\mathrm{PH}_{3}$ may be emitted by life at these detectable surface fluxes as a product of primary metabolism, such as $\mathrm{CH}_{4}$, on warm acidic worlds, or as a secondary metabolite, such as isoprene (Bains et al., 2019a, 2019b).

A surprising result of our models was that, once the phosphine surface flux reaches a tipping point $\left(\right.$ e.g., $>9 \times 10^{11}$ $\mathrm{cm}^{-2} \mathrm{~s}^{-1}$ for planets orbiting active $\mathrm{M}$ dwarfs), $\mathrm{PH}_{3}$ enters a runaway phase and begins to drastically change the atmosphere (Fig. 5). This phase appears analogous to the $\mathrm{CO}$ runaway described for early Earth and reviewed in the work of Kasting et al. (2014). In this runaway phase, $\mathrm{PH}_{3}$ production outpaces the ability of stellar NUV photons to destroy $\mathrm{PH}_{3}$ (whether via direct photolysis or via generation of radical species), and modest increases in $\mathrm{PH}_{3}$ flux lead to dramatic increases in $\mathrm{PH}_{3}$ accumulation in an atmosphere.

With fluxes of $10^{12} \mathrm{~cm}^{-2} \mathrm{~s}^{-1}$ (10 times higher than the minimum required for detection), phosphine can approach percent concentrations and would be detectable with just less than $10 \mathrm{~h}$ of observation (Table 1 and Fig. 12). In this runaway phase, $\mathrm{PH}_{3}$ can affect the concentrations of other atmospheric constituents; for example, in $\mathrm{CO}_{2}$-dominated atmospheres, the $\mathrm{H}_{2}$ concentration increases dramatically in $\mathrm{PH}_{3}$ runaway, presumably from $\mathrm{H}_{2}$ generated by $\mathrm{PH}_{3}$ destruction. This raises the possibility that enhanced $\mathrm{H}_{2}$ concentrations may be used to confirm $\mathrm{PH}_{3}$ detections. In summary, $\mathrm{PH}_{3}$ is readily detectable if it is produced at rates about an order of magnitude higher than methane or isoprene on Earth.
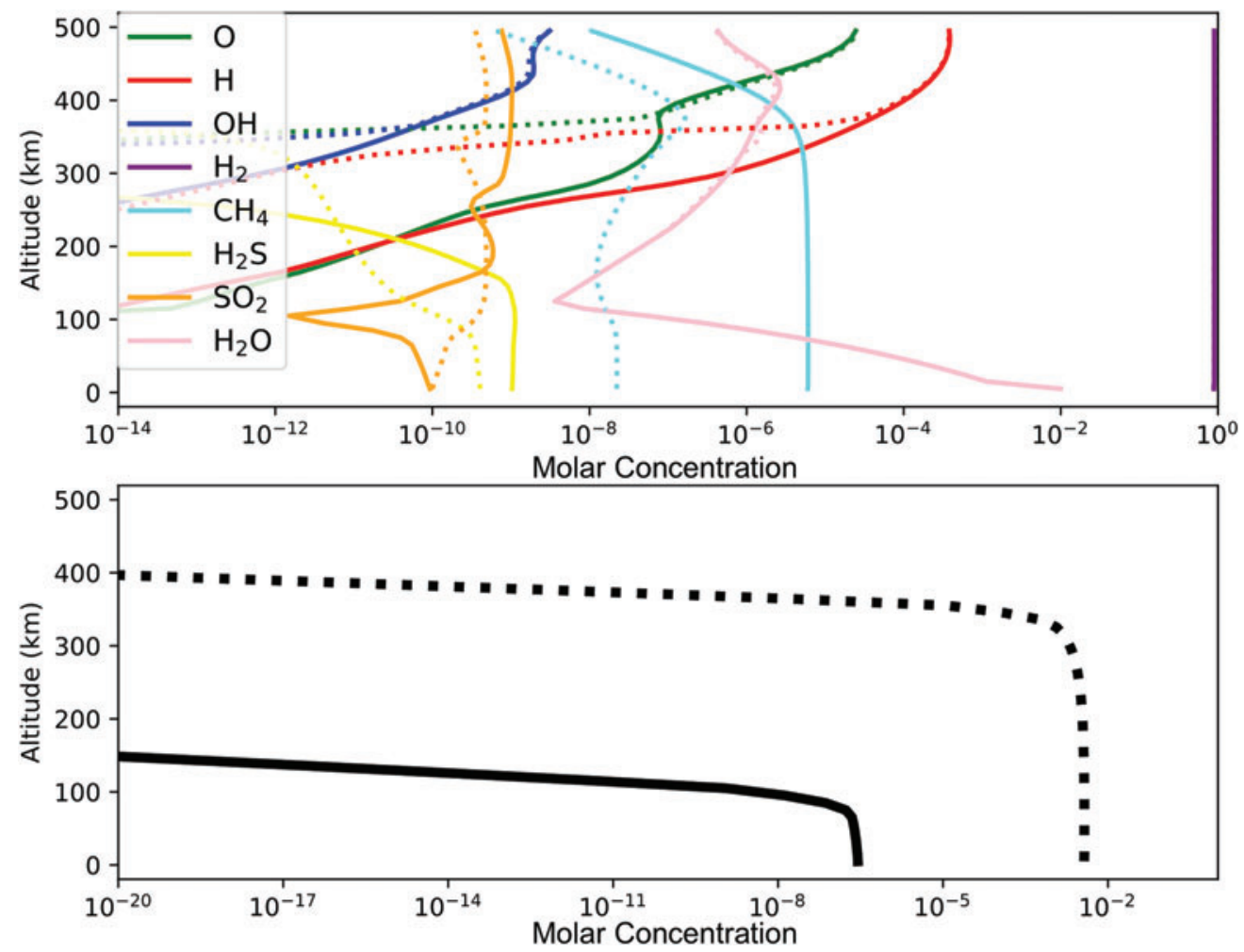

FIG. 12. Distribution of abundances of major atmospheric constituents (top panel) and phosphine (bottom panel) throughout the atmosphere of a $\mathrm{H}_{2}$-rich planet orbiting an active $\mathrm{M}$ dwarf. Solid and dashed lines show molecular abundances immediately below and above the $\mathrm{PH}_{3}$ runaway phase, respectively. The $x$ axis shows abundance concentrations, and the $y$ axis shows altitude in units of $\mathrm{km}$. The scavenging effect of $\mathrm{PH}_{3}$ leads to a decrease in $\mathrm{O}$ and $\mathrm{OH}$ and, to some extent, $\mathrm{H}$ radicals in the atmosphere. Consequently, both $\mathrm{PH}_{3}$ and other trace gases $\left(e . g ., \mathrm{H}_{2} \mathrm{~S}\right)$ are able to accumulate to larger abundances once $\mathrm{PH}_{3}$ enters the runaway phase. Color images are available online. 
Phosphine can affect the spectrum of a rocky planet atmosphere, even at concentrations somewhat below detectability, by driving down radical concentrations due to its intense reactivity with these molecules, effectively becoming a scavenger in the atmosphere. This affects the concentrations of other atmospheric constituents, such as methane. DomagalGoldman et al. (2011) described a comparable effect in models of organosulfur volatiles, which detectably altered the $\mathrm{CH}_{4}$ and $\mathrm{C}_{2} \mathrm{H}_{6}$ abundances despite being themselves undetectable. Domagal-Goldman et al. (2011) reported that elevated $\mathrm{C}_{2} \mathrm{H}_{6} /$ $\mathrm{CH}_{4}$ ratios could be diagnostic of high organosulfur flux, and hence a biosignature. Similarly, it may be possible to use the indirect effects of high $\mathrm{PH}_{3}$ flux to infer the presence of $\mathrm{PH}_{3}$ even if it is not directly detectable; more detailed measurements of the reaction kinematics of $\mathrm{PH}_{3}$ and its by-products, as well as more sophisticated atmospheric modeling are required to explore this possibility.

\subsection{Model limitations and assumptions}

We have assessed the potential of phosphine as a biosignature gas using a set of sophisticated photochemical and radiative transfer models. Nonetheless, given the complexity of simulating both atmospheric composition and subsequent spectral observations, many approximations and assumptions were made. Below is a brief discussion of the major limitations of the work performed here.

5.2.1. Clouds. We assumed cloudless skies for the simulation of exoplanet transmission spectra. To estimate how clouds might affect our results, we re-ran our models for phosphine abundances in the detectable range considering cloud decks at various altitudes, with coverage ranging from $10 \%$ to $100 \%$. As expected, the detectability of $\mathrm{PH}_{3}$ is reduced with the introduction of cloud coverage; for example, for planets with a $\mathrm{H}_{2}$-dominated atmosphere orbiting an active $\mathrm{M}$ dwarf, and with $\mathrm{PH}_{3}$ surface fluxes of $10^{11} \mathrm{~cm}^{-2}$ $\mathrm{s}^{-1}$, the detectability of $\mathrm{PH}_{3}$ reduced by up to a factor of 10 (from a cloudless model to a model with full cloud coverage at $100 \mathrm{~Pa}$ ). However, once $\mathrm{PH}_{3}$ reaches the tipping point (see Section 5.1), it becomes sufficiently abundant in the upper troposphere to be mostly unaffected by the presence of clouds.

5.2.2. Reaction networks and haze formation. In this work, we have focused on the reactivity of phosphine with the dominant radical species $\mathrm{O}, \mathrm{H}$, and $\mathrm{OH}$. The photochemistry of $\mathrm{PH}_{3}$ with radicals originating from other more exotic atmospheric species, although likely to be rare, is insufficiently studied. For example, photochemistry of $\mathrm{PH}_{3}$ and hydrocarbons, through UV radiation, could lead to the formation of complicated alkyl-phosphines (Guillemin et al., 1995, 1997), and in consequence increase the probability of hazes. Inclusion of these reactions would increase $\mathrm{PH}_{3}$ destruction rates and hence increase the required surface fluxes for detection; however, since these species are not expected to be dominant radicals, the effect of their inclusion would be minor and should not affect our results. In contrast to the formation of hydrocarbon and sulfur-based hazes that have previously been thoroughly addressed (Domagal-Goldman et al., 2011; Arney et al., 2017), there is very little work on phosphorus-based hazes. The formation of such hazes is possible in theory (Guillemin et al., 1995, 1997; Pasek et al., 2011), and early laboratory experiments implied the possibility of formation of such organophosphine hazes in planetary atmospheres, but further studies are needed to properly address the plausibility and impact of organophosphine haze formation and its associated potential as a $\mathrm{PH}_{3}$ sink. Our photochemical model will continue to update whenever we are able to expand our reaction networks.

We have neglected the formation of organic hazes in this work. There is evidence that such hydrocarbon hazes occurred on Earth, due to transient high levels of methane (Izon et al., 2017; Zahnle et al., 2019). Organic hazes are predicted to form at $\left[\mathrm{CH}_{4}\right] /\left[\mathrm{CO}_{2}\right]>0.12$ for $\mathrm{M}$ dwarf stars, and $\left[\mathrm{CH}_{4}\right] /\left[\mathrm{CO}_{2}\right] \geq 0.2$ for Sun-like stars (Arney et al., 2016, 2017). A methanogenic biosphere producing high $\mathrm{CH}_{4}$ fluxes is required to generate such high ratios; in our work, we have not considered such a biosphere. The net effect of hazes would be to facilitate phosphine buildup through attenuation of photolytic UV. However, these same hydrocarbon hazes would also cloak some of the $\mathrm{PH}_{3}$ spectral features, although primarily not in the wavelength bands where $\mathrm{PH}_{3}$ is a strong absorber.

We did not include in our models the recombination of phosphine via $\mathrm{PH}_{2}+\mathrm{H} \rightarrow \mathrm{PH}_{3}$. The rate constant for this reaction is $1.1 \times 10^{-10} \mathrm{~cm}^{3} \mathrm{~s}^{-1}$ at $288 \mathrm{~K}$ (Kaye and Strobel, 1984). The rate constant for $\mathrm{H}$ attack on $\mathrm{PH}_{3}$ is $3.3 \times 10^{-12}$ $\mathrm{cm}^{3} \mathrm{~s}^{-1}$ at $288 \mathrm{~K}$ (Arthur and Cooper, 1997). Consequently, if $\left[\mathrm{PH}_{2}\right] /\left[\mathrm{PH}_{3}\right] \geq\left(3.3 \times 10^{-12} \mathrm{~cm}^{3} \mathrm{~s}^{-1}\right) /\left(1.1 \times 10^{-10} \mathrm{~cm}^{3}\right.$ $\left.\mathrm{s}^{-1}\right)=0.03$, then reactions with $\mathrm{H}$ can reform $\mathrm{PH}_{3}$ as fast as it is destroyed by H-attack, and substantially lower the $\mathrm{PH}_{3}$ surface fluxes that are required for $\mathrm{PH}_{3}$ to accumulate in the atmosphere. Detailed photochemical modeling is required to constrain whether such high $\left[\mathrm{PH}_{2}\right] /\left[\mathrm{PH}_{3}\right]$ is plausible, or whether other sinks will suppress $\left[\mathrm{PH}_{2}\right]$. We note that, in models of Saturn's atmosphere, $\left[\mathrm{PH}_{2}\right] /\left[\mathrm{PH}_{3}\right]<0.03$ (Kaye and Strobel, 1984); if the atmospheres of terrestrial $\mathrm{H}_{2-}$ dominated exoplanets behave similarly, this recombination mechanism will not be able to significantly replenish $\mathrm{PH}_{3}$.

Finally, we note that our results reflect the prediction of atmospheric models that anoxic atmospheres should have much higher mean radical concentrations than modern Earth (Hu et al., 2012). Due to their reactivity, one might expect concentrations of radicals to be suppressed even in anoxic atmospheres, as $\mathrm{OH}$ is on Earth. While our reaction networks include all known relevant atmospheric chemistry, it is possible that there are chemical reactions, which are relevant to anoxic temperate terrestrial planets, but which have not been considered in the context of the Solar System and hence are not included in the reaction compendia we use (e.g., the NIST database (Linstrom and Mallard, 2001) and the JPL compendium (Sander et al., 2011).

Due to the incompleteness of our reaction network, it is likely that, when phosphine is destroyed, additional radicals are created that are not considered in our models. While $\mathrm{PH}_{3}$ is a trace gas, this omission should have negligible consequences, as the calculation of the infinite series due to radicals tends to converge quickly. However, when $\mathrm{PH}_{3}$ approaches the runaway phase and becomes the dominant radical sink the atmosphere, our scenarios become a lowradical regimen. In reality, the radical production would not stop, and the transition to a runaway scenario may be slower 
than we predict. If radical concentrations were overestimated in our model, then $\mathrm{PH}_{3}$ can build to detectable levels with lower surface fluxes. If, at high concentrations of $\mathrm{PH}_{3}$, the intermediate radical production from its destruction has been underestimated, then $\mathrm{PH}_{3}$ can only enter a runaway phase with higher surface fluxes than those calculated here. Detailed studies of the chemical reaction networks of anoxic planets are required to explore these possibilities.

5.2.3. Prescribed temperature-pressure profiles. Our photochemical model uses prescribed temperature-pressure profiles (Appendix A) that are isothermal above the stratosphere. In reality, this is an oversimplification; one of the consequences of assuming that there is no temperature inversion at high altitudes is the underestimation of the detectability of phosphine in our simulated emission spectra (Section 4.1.2). We also did not couple the potential heating effect from $\mathrm{PH}_{3}$ dissociation in our photochemical model, so we performed a sensitivity analysis to small changes in temperature $( \pm 15 \mathrm{~K})$ and found that our main conclusions remain unchanged (Section 4.1.4). However, if $\mathrm{PH}_{3}$ fluxes exceed the "tipping point" and $\mathrm{PH}_{3}$ enters a runaway phase, it may be possible for temperatures to increase beyond the maximum $303 \mathrm{~K}$ we consider here. In the most extreme scenario, a $\mathrm{PH}_{3}$ runaway might trigger a runaway greenhouse state, potentially rendering the planet uninhabitable. A coupled climate-photochemistry model is required to thoroughly investigate this scenario.

5.2.4. Phosphine sinks. Our photochemistry model assumes a dry deposition velocity of zero for phosphine, that is, no consumption of $\mathrm{PH}_{3}$ by surficial geochemistry or biology. Apart from its efficient oxidation by atmospheric components, there are no other known significant $\mathrm{PH}_{3}$ sinks on Earth. It is possible, however, that other planets may have $\mathrm{PH}_{3}$ deposition pathways that we cannot account for. For example, given the opportunistic nature of biology, and the fact that, at least on Earth, phosphorus is a growthlimiting nutrient, life might use any excess atmospheric $\mathrm{PH}_{3}$ as source of phosphorus. It is plausible that anaerobic life on other planets will not only just produce $\mathrm{PH}_{3}$ but also reabsorb it from the atmosphere; in these scenarios, biology would slow down the $\mathrm{PH}_{3}$ accumulation in the atmosphere leading to a dampening, and possible avoidance, of a runaway $\mathrm{PH}_{3}$ effect (Section 5.1). In extreme cases, for example, if biological production of $\mathrm{PH}_{3}$ can equal its reabsorption, life's recycling of biogenic $\mathrm{PH}_{3}$ might entirely prevent its accumulation in the atmosphere of an exoplanet.

To estimate the effect of a potential sink for phosphine, we tested the variability of our results to a non-zero deposition velocity for $\mathrm{PH}_{3}$. Deposition velocity tests show that, for a comparable deposition rate to $\mathrm{CO}$ and $\mathrm{O}_{2}\left(10^{-4} \mathrm{~cm}^{-1}\right)$ (Harman et al., 2015), concentrations of $\mathrm{PH}_{3}$ varied by a factor of $<2$, which is not enough to affect detectability. We note that this is a conservative estimate given that the only plausible $\mathrm{PH}_{3}$ sinks are biological.

5.2.5. Phosphorus availability. We considered whether the availability of phosphorus in the crust of a planet could be a limiting factor for the accumulation of phosphine in the atmosphere. An estimate of the total phosphorus within Earth's crust shows that, if all the phosphorus was to be converted to $\mathrm{PH}_{3}$, it would produce approximately twice as many $\mathrm{PH}_{3}$ molecules as the total number of all molecules belonging to all gases present in the atmosphere of the modern Earth (Yaroshevsky, 2006). We conclude that, in principle, the total mass of phosphorus in a planetary crust does not limit the development of a high- $\mathrm{PH}_{3}$ atmosphere.

\subsection{Alternative detection methods beyond JWST}

For the phosphine detectability calculations in this work, we have considered observations from a JWST-like telescope, with a $6.5 \mathrm{~m}$ diameter telescope mirror operating within $50 \%$ of the shot noise limit and a quantum efficiency between $20 \%$ and $25 \%$. The integration time is assumed to be under $200 \mathrm{~h}$ for all atmospheric scenarios. For comparison, the cryogenic lifetime of JWST is 5 years, which is equivalent to an integration time of $100 \mathrm{~h}$ for a planet orbiting the habitable zone of an $\mathrm{M}$ dwarf star. The spectral resolution of JWST is $R=100$ at $1-5 \mu \mathrm{m}$ and $R=160$ at $5-12 \mu \mathrm{m}$, which is more than necessary for distinguishing between $\mathrm{PH}_{3}$ and other dominant gases in the atmosphere (see Section 4.2).

We considered the possibility of detecting phosphine in anoxic atmospheres using alternative telescopes to JWST. Missions such as TPF-I (Lawson et al., 2008), Darwin (Fridlund, 2000), Origins Space Telescope (OST) (Battersby et al., 2018), Habitable Exoplanet Imaging Mission (HabEx) (Gaudi et al., 2018), Large UV Optical Infrared Surveyor (LUVOIR) (Roberge, 2019), and the 30-m class of ground telescopes (Johns et al., 2012; Tamai and Spyromilio, 2014; Skidmore et al., 2015) could also be able to characterize atmospheres of temperate planets in wavelength regions where $\mathrm{PH}_{3}$ is spectrally active.

TPF-I was intended to be a nulling interferometer with four $4 \mathrm{~m}$ diameter telescopes flying in formation with a baseline range of 40-100 $\mathrm{m}$ and operating at $6.5-18 \mu \mathrm{m}$ with a spectral resolution of 25-50. Darwin was planned as set of 3-4 $\mathrm{m}$ diameter telescopes flying in a nulling interferometer configuration. For both instruments, only the nearest $(\sim 4$ pc) M dwarf star habitable zones would be accessible. Winters et al. (2019) estimated that there are only $22 \mathrm{M}$ dwarf stars candidates that could have planets within their habitable zone suitable for atmospheric characterization. TPF-I and Darwin are currently canceled, but similar telescopes [e.g., the proposed Large Interferometer For Exoplanets, or LIFE (Quanz et al., 2019)] may one day be commissioned that perform in a similar interferometer formation and could have the capability to identify phosphine on temperate exoplanets orbiting $\mathbf{M}$ dwarfs.

The OST (Battersby et al., 2018) has a large wavelength coverage $(2.8-20 \mu \mathrm{m})$ and will be able to provide atmospheric spectra for planets orbiting $\mathrm{K}$ and $\mathrm{M}$ dwarf stars, through transmission and secondary eclipse observations. The OST will have the sensitivity and coverage to detect many spectral signatures of potential biosignature gases, including phosphine.

The HabEx (Gaudi et al., 2018) and the LUVOIR (Roberge, 2019) are planned as powerful telescopes that could launch in the coming decades and are focused on the detection of potential biosignature gases. Both cover a wavelength region where phosphine is spectrally active (UV to near-infrared), although not a particularly strong absorber $(1.28-1.79 \mu \mathrm{m})$ (Sousa-Silva et al., 2014). We have not 
modeled atmospheres with $\mathrm{PH}_{3}$ in an HabEx/LUVOIR scenario, but these telescopes' high-contrast spectroscopy may allow for some of the high-frequency combination and hot bands of $\mathrm{PH}_{3}$ to be detected.

Future $30-\mathrm{m}$ ground-based telescopes will be limited by Earth's observing windows, but those capable of $\mathrm{M}$ - and N-band spectroscopy (4.5-5.0 and 7.5-14.5 $\mu \mathrm{m}$, respectively) would be favorable for the detection of phosphine, given its strong feature at $4-4.8 \mu \mathrm{m}$ and its broad band centered at $10 \mu \mathrm{m}$ (see Section 4.2).

\subsection{Phosphine as a biosignature gas}

An ideal biosignature gas lacks abiotic false positives, has uniquely identifiable spectral features, and is unreactive enough to build up to detectable concentrations in exoplanet atmospheres. Phosphine fulfills the first two criteria: $\mathrm{PH}_{3}$ is only known to be associated with life and geochemical false positives for $\mathrm{PH}_{3}$ generation are highly unlikely (Bains et al., 2017, 2019a); $\mathrm{PH}_{3}$ possesses three strong features in the $2.7-3.6,4-4.8$, and $7.8-11 \mu \mathrm{m}$ regions that are distinguishable from common outgassed species that may be present in terrestrial exoplanet atmospheres, such as $\mathrm{CO}_{2}$, $\mathrm{H}_{2} \mathrm{O}, \mathrm{CO}, \mathrm{CH}_{4}, \mathrm{NH}_{3}$, and $\mathrm{H}_{2} \mathrm{~S}$.

The greatest challenge to the detectability of phosphine at low surface fluxes is its reactivity to radicals and its vulnerability to UV photolysis. We found that, in the most tractable observational scenario (planet orbiting an active $\mathrm{M}$ dwarf), $\mathrm{PH}_{3}$ must be emitted at a rate of $10^{11} \mathrm{~cm}^{-2} \mathrm{~s}^{-1}$ to build to levels detectable by transmission or thermal emission spectroscopy (e.g., using JWST). The required $\mathrm{PH}_{3}$ production rates for detection are two orders of magnitude lower for planets orbiting a hypothetical "quiet" M dwarf with extremely low levels of chromospheric activity; this latter scenario is likely unrealistic and corresponds to an extreme lower limit on the required $\mathrm{PH}_{3}$ flux for its detection. An alternative path for a UV-poor surface environment would be a planet with a UV shield, or possibly intense hazes, although hazes might also inhibit a $\mathrm{PH}_{3}$ detection. Finally, our models suggest that, at high but plausible surface fluxes $\left(10^{12}-10^{14} \mathrm{~cm}^{-2} \mathrm{~s}^{-1}\right.$, depending on the planetary scenario), $\mathrm{PH}_{3}$ is able to exhaust the supply of $\mathrm{M}$ dwarf NUV photons and enters a "runaway" phase. If such a runaway effect occurs, $\mathrm{PH}_{3}$ becomes easily detectable, protects other trace gases from destruction by radicals, and rapidly changes the overall composition of the planetary atmosphere.

We compare phosphine with another proposed biosignature gas: methyl chloride or $\mathrm{CH}_{3} \mathrm{Cl}$ (Segura et al., 2005). The surface flux required for $\mathrm{CH}_{3} \mathrm{Cl}$ buildup to detectable levels is $5 \times 10^{7}$ to $3 \times 10^{11} \mathrm{~cm}^{-2} \mathrm{~s}^{-1}$ under the same atmospheric scenarios we consider here, or up to four orders of magnitude less than $\mathrm{PH}_{3}$. This difference is rooted in the lower reactivity of $\mathrm{CH}_{3} \mathrm{Cl}$ with radicals; its reaction rates with $\mathrm{O}$ and $\mathrm{H}$ are four to six orders of magnitude lower than $\mathrm{PH}_{3}$ (Seager et al., 2013b). We conclude that it is more difficult for $\mathrm{PH}_{3}$ to build to detectable concentrations than $\mathrm{CH}_{3} \mathrm{Cl}$ because of its higher reactivity with radicals. As a further comparison, the modern methane surface flux is 535 $\mathrm{Tg}$ /year $=1.2 \times 10^{11} \mathrm{~cm}^{-2} \mathrm{~s}^{-1}$, meaning that for $\mathrm{PH}_{3}$ to build to detectable levels in planets orbiting active stars, it must be emitted at a rate comparable to that of methane on Earth
(Houghton, 1995; Segura et al., 2005; Guzmán-Marmolejo and Segura, 2015).

For a final comparison, we consider the highest concentrated fluxes of phosphine on Earth. On Earth, detections of $\mathrm{PH}_{3}$ in biogas from sewage plants can reach very high levels, for example, $10^{14} \mathrm{~cm}^{-2} \mathrm{~s}^{-1}$ (Devai et al., 1988). It is therefore plausible that a complex anaerobic biosphere, not dissimilar from those found in sludges on Earth, could achieve global $\mathrm{PH}_{3}$ production levels comparable to the production rates found locally in isolated environments on Earth. In such anoxic environments, $\mathrm{PH}_{3}$ would be detectable in transmission and emission for planets orbiting $M$ dwarfs (Table 1). In other words, we can imagine, for example, a planet like Earth in the early Carboniferous period ( $\sim 318$ Mya), but without $\mathrm{O}_{2}$ in the atmosphere: a wet anoxic "tropical paradise" from pole to pole. Such a planet could potentially produce tremendous amounts of $\mathrm{PH}_{3}$ by its rich anaerobic biosphere.

Phosphine's relevance as a biosignature gas depends on its production by life, on its possible geochemical false positives, and on its detectability. Prospects for the buildup of $\mathrm{PH}_{3}$ to detectable levels are uncertain with near-future telescopes. However, the wavelength regions where $\mathrm{PH}_{3}$ is spectrally active are similar to those of other atmospherically important molecules, for example, $\mathrm{H}_{2} \mathrm{O}$ and $\mathrm{CH}_{4}$. Consequently, searches for $\mathrm{PH}_{3}$ can be carried out at no additional observational cost to searches for other molecular species relevant to characterizing exoplanet habitability.

Ultimately, if detected on a temperate planet, phosphine is an extremely promising biosignature gas, since its lack of high-flux false positives would be a strong reason to hypothesize production by life.

\section{Acknowledgments}

We thank the MIT BOSE Fellow program and the Change Happens Foundation for partial funding of this work. We thank Elisabeth Matthews, Thomas Evans, Julien de Wit, and Jason Dittmann for their advice on detectability metrics. We also thank Antonio P. Silva, Fionnuala Cavanagh, Catherine Wilka, Sarah Ballard, Sarah Rugheimer, Jennifer Burt, Daniel Koll, Susan Solomon, Andrew Babbin, Tiffany Kataria, Antonio Silva, and Christopher Shea for their support and useful discussions. Finally, we would like to thank our two reviewers, whose contributions significantly improved this article.

\section{Author Disclosure Statement}

No competing financial interests exist.

\section{Funding Information}

This research was supported in part by grants from the Simons Foundation (SCOL; Grant No. 495062 to S.R.), the Heising-Simons Foundation (51 Pegasi b Fellowship to C.SS.), and carried out in part at the Jet Propulsion Laboratory, California Institute of Technology, under a contract with the National Aeronautics and Space Administration.

\section{References}

Agúndez, M., Cernicharo, J., Decin, L., Encrenaz, P., and Teyssier, D. (2014) Confirmation of circumstellar phosphine. Astrophys J Lett 790:L27.

Arney, G., Domagal-Goldman, S.D., Meadows, V.S., Wolf, E.T., Schwieterman, E., Charnay, B., Claire, M., Hébrard, E., and 
Trainer, M.G. (2016) The pale orange dot: the spectrum and habitability of hazy Archean Earth. Astrobiology 16:873-899. Arney, G.N., Meadows, V.S., Domagal-Goldman, S.D., Deming, D., Robinson, T.D., Tovar, G., Wolf, E.T., and Schwieterman, E. (2017) Pale orange dots: the impact of organic haze on the habitability and detectability of Earthlike exoplanets. Astrophys $J$ 836:49.

Arthur, N.L. and Cooper, I.A. (1997) Arrhenius parameters for the reactions of $\mathrm{H}$ atoms with $\mathrm{PH} 3$ and AsH3. J Chem Soc Faraday Trans 93:521-524.

Bagnasco, G., Kolm, M., Ferruit, P., Honnen, K., Koehler, J., Lemke, R., Maschmann, M., Melf, M., Noyer, G., and Rumler, P. (2007) Overview of the Near-Infrared Spectrograph (NIRSpec) Instrument On-Board the James Webb Space Telescope (JWST). International Society for Optics and Photonics, Bellingham, WA.

Bains, W., Petkowski, J.J., and Seager, S. (2017) Toward a list of molecules as potential biosignature gases for the search for life on exoplanets: thermodynamic profiling potential false positives. In: Astrobiology Science Conference (AbSciCon), Mesa, Arizona.

Bains, W., Petkowski, J.J., Sousa-Silva, C., and Seager, S. (2019a) New environmental model for thermodynamic ecology of biological phosphine production. Sci Total Environ 658:521-536.

Bains, W., Petkowski, J.J., Sousa-Silva, C., and Seager, S. (2019b) Trivalent phosphorus and phosphines as components of biochemistry in anoxic environments. Astrobiology 19: 885-902.

Batalha, N., Kalirai, J., Lunine, J., Clampin, M., and Lindler, D.J. (2015) Transiting exoplanet simulations with the James Webb Space Telescope. arXiv:1507.02655. Available online at http://arxiv.org/abs/1507.02655

Battersby, C., Armus, L., Bergin, E., Kataria, T., Meixner, M., Pope, A., Stevenson, K.B., Cooray, A., Leisawitz, D., and Scott, D. (2018) The Origins Space Telescope. Nat Astron 2:596.

Bera, P.P., Francisco, J.S., and Lee, T.J. (2009) Identifying the molecular origin of global warming. J Phys Chem A 113: 12694-12699.

Berk, A., Bernstein, L.S., Anderson, G.P., Acharya, P.K., Robertson, D.C., Chetwynd, J.H., and Adler-Golden, S.M. (1998) MODTRAN cloud and multiple scattering upgrades with application to AVIRIS. Remote Sens Environ 65:367-375.

Bernath, P.F., McElroy, C.T., Abrams, M.C., Boone, C.D., Butler, M., Camy-Peyret, C., Carleer, M., Clerbaux, C., Coheur, P.F., and Colin, R. (2005) Atmospheric chemistry experiment (ACE): mission overview. Geophys Res Lett 32:L15S01.

Bingham, E. (2001) Phosphorus, selenium, tellurium and sulfur. In: Patty's Toxicology, 5th ed, Vol. 3, edited by E. Bingham, B. Cohrssen, and C.H. Powells, John Wiley, New York, NY, pp 459-517.

Bregman, J.D., Lester, D.F., and Rank, D.M. (1975) Observation of the nu-squared band of $\mathrm{PH} 3$ in the atmosphere of Saturn. Astrophys J 202:L55-L56.

Brown, T.M. (2001) Transmission spectra as diagnostics of extrasolar giant planet atmospheres. Astrophys $J$ 553:1006.

Budavari, S., O'Neil, M.J., Smith, A., Heckelman, P.E., and Kinneary, J.F. (1996) The Merck Index. Merck Research Laboratories Division of Merck \& Co., Inc., Kenilworth, NJ.

Burgdorf, M.J., Orton, G.S., Encrenaz, T., Davis, G.R., Lellouch, E., Sidher, S.D., and Swinyard, B.M. (2004) Farinfrared spectroscopy of the giant planets: measurements of ammonia and phosphine at Jupiter and Saturn and the continuum of Neptune. Adv Space Res 34:2247-2250.
Cao, H., Liu, J.A., Zhuang, Y., and Dietmar, G. (2000) Emission sources of atmospheric phosphine and simulation of phosphine formation. Sci China Ser B Chem 43:162-168.

Carlson, R.W., Baines, K.H., Anderson, M.S., Filacchione, G., and Simon, A.A. (2016) Chromophores from photolyzed ammonia reacting with acetylene: application to Jupiter's Great Red Spot. Icarus 274:106-115.

Caspi, R., Billington, R., Fulcher, C.A., Keseler, I.M., Kothari, A., Krummenacker, M., Latendresse, M., Midford, P.E., Ong, Q., Ong, W.K., Paley, S., Subhraveti, P., Karp, P.D. (2017) The MetaCyc database of metabolic pathways and enzymes. Nucleic Acids Res 46:D633-D639.

Chen, F., Judge, D.L., Robert Wu, C.Y., Caldwell, J., White, H.P., and Wagener, R. (1991) High-resolution, lowtemperature photoabsorption cross sections of $\mathrm{C} 2 \mathrm{H} 2, \mathrm{PH} 3$, AsH3, and GeH4, with application to Saturn's atmosphere. $J$ Geophys Res Planets 96:17519-17527.

Chen, W., Niu, X., An, S., Sheng, H., Tang, Z., Yang, Z., and $\mathrm{Gu}, \mathrm{X}$. (2017) Emission and distribution of phosphine in paddy fields and its relationship with greenhouse gases. Sci Total Environ 599-600:952-959.

Chughtai, M. and Pridham, J.B. (1998) Determination of phosphine by packed column gas chromatography with alkali flame ionisation detection. Anal Commun 35:109-111.

Devai, I. and Delaune, R.D. (1995) Evidence for phosphine production and emission from Louisiana and Florida marsh soils. Org Geochem 23:277-279.

Devai, I., Felfoldy, L., Wittner, I., and Plosz, S. (1988) Detection of phosphine: new aspects of the phosphorus cycle in the hydrosphere. Nature 333:343-345.

Ding, L., Liang, H., Zhu, Y., Mo, W., Wang, Q., Ren, H., Wang, X., Edwards, M., and Glindemann, D. (2005a) Sources of matrix-bound phosphine in advanced wastewater treatment system. Chinese Sci Bull 50:1274-1276.

Ding, L., Wang, X., Zhu, Y., Edwards, M., Glindemann, D., and Ren, H. (2005b) Effect of $\mathrm{pH}$ on phosphine production and the fate of phosphorus during anaerobic process with granular sludge. Chemosphere 59:49-54.

Ding, W., Zhu, R., Hou, L., and Wang, Q. (2014) Matrix-bound phosphine, phosphorus fractions and phosphatase activity through sediment profiles in Lake Chaohu, China. Environ Sci Processes Impacts 16:1135-1144.

Domagal-Goldman, S.D., Meadows, V.S., Claire, M.W., and Kasting, J.F. (2011) Using biogenic sulfur gases as remotely detectable biosignatures on anoxic planets. Astrobiology 11: 419-441.

Eismann, F., Glindemann, D., Bergmann, A., and Kuschk, P. (1997a) Balancing phosphine in manure fermentation. $J$ Environ Sci Health B 32:955-968.

Eismann, F., Glindemann, D., Bergmannt, A., and Kuschk, P. (1997b) Soils as source and sink of phosphine. Chemosphere 35:523-533.

Elm, J., Myllys, N., and Kurtén, T. (2017) Phosphoric acid-a potentially elusive participant in atmospheric new particle formation. Mol Phys 115:2168-2179.

Emiliani, C. (1992) Planet Earth: Cosmology, Geology, and the Evolution of Life and Environment. Cambridge University Press, Cambridge, UK.

Fan, Yimin, et al. (2020) "Evidence and mechanism of biological formation of phosphine from the perspective of the tricarboxylic acid cycle." International Biodeterioration \& Biodegradation 146:104791.

Feng, Z., Song, X., and Yu, Z. (2008) Seasonal and spatial distribution of matrix-bound phosphine and its relationship 
with the environment in the Changjiang River Estuary, China. Mar Pollut Bull 56:1630-1636.

Fletcher, L.N., Orton, G.S., Teanby, N.A., and Irwin, P.G.J. (2009) Phosphine on Jupiter and Saturn from Cassini/CIRS. Icarus 202:543-564.

Fluck, E. (1973) The Chemistry of Phosphine. Fortschritte der Chemischen Forschung (Inorganic Chemistry) 35:1-64.

France, K., Froning, C.S., Linsky, J.L., Roberge, A., Stocke, J.T., Tian, F., Bushinsky, R., Désert, J.-M., Mauas, P., and Vieytes, M. (2013) The ultraviolet radiation environment around M dwarf exoplanet host stars. Astrophys $J$ 763:149.

France, K., Loyd, R.P., Youngblood, A., Brown, A., Schneider, P.C., Hawley, S.L., Froning, C.S., Linsky, J.L., Roberge, A., and Buccino, A.P. (2016) The MUSCLES treasury survey. I. Motivation and overview. Astrophys J 820:89.

Frank, R., and Rippen, G. (1987) Verhalten von phosphin in der Atmosphäre [Fate of phosphine in the atmosphere]. Lebensmitteltechnik [Food Technol] 17:409-411.

Fridlund, C.V.M. (2000) Darwin-the infrared space interferometry mission. ESA Bull 103:20-25.

Fritz, B., Lorenz, K., Steinert, W., and Zellner, R. (1982) Laboratory kinetic investigations of the tropospheric oxidation of selected industrial emissions. Phys Chem Behav Atmos Pollut Proc Eur Symp.

Fu, M., Yu, Z., Lu, G., and Song, X. (2013) Henry's law constant for phosphine in seawater: determination and assessment of influencing factors. Chin J Oceanol Limnol 31:860-866.

Gardner, J.P., Mather, J.C., Clampin, M., Doyon, R., Greenhouse, M.A., Hammel, H.B., Hutchings, J.B., Jakobsen, P., Lilly, S.J., Long, K.S., Lunine, J.I., McCaughrean, M.J., Mountain, M., Nella, J., Rieke, G.H., Rieke, M.J., Rix, H.W., Smith, E.P., Sonneborn, G., Stiavelli, M., Stockman, H.S., Windhorst, R.A., and Wright, G.S. (2006) The James Webb Space Telescope. Space Sci Rev 123:485-606.

Gassmann, G. (1994) Phosphine in the fluvial and marine hydrosphere. Mar Chem 45:197-205.

Gassmann, G. and Glindemann, D. (1993) Phosphane (PH3) in the biosphere. Angew Chem Int Ed Engl 32:761-763.

Gassmann, G., Van Beusekom, J.E.E., and Glindemann, D. (1996) Offshore atmospheric phosphine. Naturwissenschaften [The Science of Nature]. 83:129-131.

Gaudi, B.S., Seager, S., Mennesson, B., Kiessling, A., Warfield, K., Kuan, G., Cahoy, K., Clarke, J.T., Domagal-Goldman, S., and Feinberg, L. (2018) The Habitable Exoplanet Observatory (HabEx) mission concept study interim report. $a r-$ Xiv Preprint arXiv: 1809.09674.

Geist, V., Wagner, G., Nolze, G., and Moretzki, O. (2005) Investigations of the meteoritic mineral $(\mathrm{Fe}, \mathrm{Ni}) 3 \mathrm{P}$. Cryst Res Technol 40:52-64.

Geng, J., Niu, X., Jin, X., Wang, X., Gu, X., Edwards, M., and Glindemann, D. (2005) Simultaneous monitoring of phosphine and of phosphorus species in Taihu lake sediments and phosphine emission from lake sediments. Biogeochemistry 76:283-298.

Geng, J., Niu, X., Wang, X., Edwards, M., and Glindemann, D. (2010) The presence of trace phosphine in Lake Taihu water. Int J Environ Anal Chem 90:737-746.

Glindemann, D., Bergmann, A., Stottmeister, U., and Gassmann, G. (1996a) Phosphine in the lower terrestrial troposphere. Naturwissenschaften [The Science of Nature]. 83: 131-133.

Glindemann, D., Stottmeister, U., and Bergmann, A. (1996b) Free phosphine from the anaerobic biosphere. Environ Sci Pollut Res 3:17-19.
Glindemann, D., Eismann, F., Bergmann, A., Kuschk, P., and Stottmeister, U. (1998) Phosphine by bio-corrosion of phosphide-rich iron. Environ Sci Pollut Res 5:71-74.

Glindemann, D., de Graaf, R.M., and Schwartz, A.W. (1999) Chemical reduction of phosphate on the primitive Earth. Orig Life Evol Biosph 29:555-561.

Glindemann, D., Edwards, M., and Kuschk, P. (2003) Phosphine gas in the upper troposphere. Atmosph Environ 37:2429-2433.

Glindemann, D., Edwards, M., and Schrems, O. (2004) Phosphine and methylphosphine production by simulated lightning-a study for the volatile phosphorus cycle and cloud formation in the earth atmosphere. Atmos Environ 38:6867-6874.

Glindemann, D., Edwards, M., Liu, J.-A., and Kuschk, P. (2005) Phosphine in soils, sludges, biogases and atmospheric implications-a review. Ecol Eng 24:457-463.

Gordon, I.E., Rothman, L.S., Hill, C., Kochanov, R.V., Tan, Y., Bernath, P.F., Birk, M., Boudon, V., Campargue, A., and Chance, K.V. (2017) The HITRAN2016 molecular spectroscopic database. J Quant Spectrosc Radiat Transfer 203: 3-69.

Grenfell, J.L. (2018) Atmospheric Biosignatures. In: Handbook of Exoplanets, edited by H.J. Deeg and J.A. Belmontes, Springer International Publishing, Cham, pp 1-14.

Guenther, A., Karl, T., Harley, P., Wiedinmyer, C., Palmer, P.I., and Geron, C. (2006) Estimates of global terrestrial isoprene emissions using MEGAN (Model of Emissions of Gases and Aerosols from Nature). Atmos Chem Phys 6: 3181-3210.

Guillemin, J.C., Janati, T., and Lassalle, L. (1995) Photolysis of phosphine in the presence of acetylene and propyne, gas mixtures of planetary interest. Adv Space Res 16:85-92.

Guillemin, J.-C., Le Serre, S., and Lassalle, L. (1997) Regioselectivity of the photochemical addition of phosphine to unsaturated hydrocarbons in the atmospheres of Jupiter and Saturn. Adv Space Res 19:1093-1102.

Guzmán-Marmolejo, A., and Segura, A. (2015) Methane in the solar system. Boletín de la Sociedad Geológica Mexicana [The Bulletin of the Mexican Geological Society]. 67:377-385.

Han, C., Geng, J., Hong, Y., Zhang, R., Gu, X., Wang, X., Gao, S., and Glindemann, D. (2011a) Free atmospheric phosphine concentrations and fluxes in different wetland ecosystems, China. Environ Pollut 159:630-635.

Han, C., Geng, J., Zhang, J., Wang, X., and Gao, S. (2011b) Phosphine migration at the water-air interface in Lake Taihu, China. Chemosphere 82:935-939.

Han, C., Geng, J., Xie, X., Wang, X., Ren, H., and Gao, S. (2012) Determination of phosphite in a eutrophic freshwater lake by suppressed conductivity ion chromatography. Environ Sci Technol 46:10667-10674.

Han, C., Geng, J., Ren, H., Gao, S., Xie, X., and Wang, X. (2013) Phosphite in sedimentary interstitial water of Lake Taihu, a large eutrophic shallow lake in China. Environ Sci Technol 47:5679-5685.

Han, S.-H., Zhuang, Y.-H., Liu, J.-A., and Glindemann, D. (2000) Phosphorus cycling through phosphine in paddy fields. Sci Total Environ 258:195-203.

Harman, C.E., Schwieterman, E.W., Schottelkotte, J.C., and Kasting, J.F. (2015) Abiotic O2 levels on planets around F, G, $\mathrm{K}$, and $\mathrm{M}$ stars: possible false positives for life? Astrophys $\boldsymbol{J}$ 812:137.

Herschy, B., Chang, S.J., Blake, R., Lepland, A., Abbott-Lyon, H., Sampson, J., Atlas, Z., Kee, T.P., and Pasek, M.A. (2018) Archean phosphorus liberation induced by iron redox geochemistry. Nat Commun 9:1346. 
Hill, C., Yurchenko, S.N., and Tennyson, J. (2013) Temperaturedependent molecular absorption cross sections for exoplanets and other atmospheres. Icarus 226:1673-1677.

Holland, H.D. (1984) The Chemical Evolution of the Atmosphere and Oceans. Princeton University Press, Princeton, NJ.

Hong, Y., Geng, J., Qiao, S., Ding, L., Gu, X., Wang, X., Glindemann, D., and Ren, H. (2010a) Distribution of phosphine in the offshore area of the Southwest Yellow Sea, East Asia. Mar Chem 118:67-74.

Hong, Y., Geng, J., Qiao, S., Zhang, Y., Ding, L., Wang, X., and Ren, H. (2010b) Phosphorus fractions and matrix-bound phosphine in coastal surface sediments of the Southwest Yellow Sea. J Hazard Mater 181:556-564.

Houghton, J.T. (1995) Climate Change 1994: Radiative Forcing of Climate Change and an Evaluation of the IPCC 1992 IS92 Emission Scenarios. Cambridge University Press, Cambridge.

$\mathrm{Hu}, \mathrm{R}$. and Seager, S. (2014) Photochemistry in terrestrial exoplanet atmospheres. III. Photochemistry and thermochemistry in thick atmospheres on super Earths and mini Neptunes. Astrophys $J$ 784:63.

$\mathrm{Hu}$, R., Seager, S., and Bains, W. (2012) Photochemistry in terrestrial exoplanet atmospheres. I. Photochemistry model and benchmark cases. Astrophys J 761:166.

$\mathrm{Hu}$, R., Seager, S., and Bains, W. (2013) Photochemistry in terrestrial exoplanet atmospheres. II. H2S and SO2 photochemistry in anoxic atmospheres. Astrophys $J$ 769:6.

Izon, G., Zerkle, A.L., Williford, K.H., Farquhar, J., Poulton, S.W., and Claire, M.W. (2017) Biological regulation of atmospheric chemistry en route to planetary oxygenation. Proc Natl Acad Sci U S A 114:E2571-E2579.

Jacob, D.J. (1999) Introduction to Atmospheric Chemistry. Princeton University Press, Princeton, NJ.

Jenkins, R.O., Morris, T.A., Craig, P.J., Ritchie, A.W., and Ostah, N. (2000) Phosphine generation by mixed- and monosepticcultures of anaerobic bacteria. Sci Total Environ 250:73-81.

Ji-ang, L., Yahui, C.H.Z., Kuschk, P., Eismann, F., and Glindemann, D. (1999) Phosphine in the urban air of Beijing and its possible sources. Water Air Soil Pollut 116:597-604.

Johns, M., McCarthy, P., Raybould, K., Bouchez, A., Farahani, A., Filgueira, J., Jacoby, G., Shectman, S., and Sheehan, M. (2012) Giant Magellan Telescope: overview. SPIE Astronomical Telescopes+Instrumentation. SPIE, Bellingham, WA.

Kasting, J.F. (1993) Earth's early atmosphere. Science 259:920926.

Kasting, J.F. (2014) Atmospheric composition of Hadean-early Archean Earth: the importance of CO. Geol Soc Am Spec Pap 504:19-28.

Kasting, J.F., Zahnle, K.J., and Walker, J.C.G. (1983) Photochemistry of methane in the Earth's early atmosphere. Precambrian Res 20:121-148.

Kasting, J.F., Pollack, J.B., and Crisp, D. (1984) Effects of high $\mathrm{CO} 2$ levels on surface temperature and atmospheric oxidation state of the early Earth. J Atmos Chem 1:403-428.

Kasting, J.F., Kopparapu, R., Ramirez, R.M., and Harman, C.E. (2014) Remote life-detection criteria, habitable zone boundaries, and the frequency of Earth-like planets around $\mathrm{M}$ and late K stars. Proc Natl Acad Sci U S A 111:12641.

Kaye, J.A., and Strobel, D.F. (1984) Phosphine photochemistry in the atmosphere of Saturn. Icarus 59:314-335.

Keller-Rudek, H., Moortgat, G.K., Sander, R., and Sörensen, R. (2013) The MPI-Mainz UV/VIS spectral atlas of gaseous molecules of atmospheric interest. Earth Syst Sci Data 5:365-373.

Kempton, E.M.R., Lupu, R., Owusu-Asare, A., Slough, P., and Cale, B. (2017) Exo-Transmit: an open-source code for cal- culating transmission spectra for exoplanet atmospheres of varied composition. Publ Astron Soc Pac 129:044402.

Kiang, N.Y., Domagal-Goldman, S., Parenteau, M.N., Catling, D.C., Fujii, Y., Meadows, V.S., Schwieterman, E.W., and Walker, S.I. (2018) Exoplanet Biosignatures: at the dawn of a new era of planetary observations. Astrobiology 18:619-629.

Kim, S.-J. (1996) Phosphine and Jupiter's great red spot. $J$ Astron Space Sci 13:32-39.

Kochanov, R.V., Gordon, I.E., Rothman, L.S., Wcisło, P., Hill, C., and Wilzewski, J.S. (2016) HITRAN Application Programming Interface (HAPI): a comprehensive approach to working with spectroscopic data. J Quant Spectrosc Radiat Transfer 177:15-30.

Lawson, P., Lay, O., Martin, S., Peters, R., Gappinger, R., Ksendzov, A., Scharf, D., Booth, A., Beichman, C., and Serabyn, E. (2008) Terrestrial Planet Finder Interferometer: 2007-2008 Progress and Plans.Optical and Infrared Interferometry. International Society for Optics and Photonics, Bellingham, WA.

Lemmon, E.W., Huber, M.L., and McLinden, M.O. (2010) NIST Standard Reference Database 23. NIST reference fluid thermodynamic and transport properties-REFPROP, version 9, 55.

Lewis, W.M., Grant, M.C., and Hamilton, S.K. (1985) Evidence that filterable phosphorus is a significant atmospheric link in the phosphorus cycle. Oikos 45:428-432.

Li, J.-B., Zhang, G.-L., Zhang, J., Liu, S.-M., Ren, J.-L., and Hou, Z.-X. (2009) Phosphine in the lower atmosphere of Qingdao-a coastal site of the Yellow Sea (China). Water Air Soil Pollut 204:117-131.

Linstrom, P.J., and Mallard, W.G. (2001) The NIST Chemistry WebBook: a chemical data resource on the Internet. $J$ Chem Eng Data 46:1059-1063.

Liu, Z., Jia, S., Wang, B., Zhang, T., and Liu, S. (2008) Preliminary investigation on the role of microorganisms in the production of phosphine. J Environ Sci 20:885-890.

Martin, W., Baross, J., Kelley, D., and Russell, M.J. (2008) Hydrothermal vents and the origin of life. Nat Rev Microbiol 6:805.

Miller-Ricci, E., Meyer, M.R., Seager, S., and Elkins-Tanton, L. (2009) On the emergent spectra of hot protoplanet collision afterglows. Astrophys J 704:770.

Moreno, R., Marten, A., and Lellouch, E. (2009) Search for PH3 in the atmospheres of Uranus and Neptune at millimeter wavelength. Am Astron Soc DPS Meeting \#41, 41:id.28.02, Volume 41.

Morton, S.C. and Edwards, M. (2005) Reduced phosphorus compounds in the environment. Crit Rev Environ Sci Technol 35:333-364.

Moss, A.R., Jouany, J.-P., and Newbold, J. (2000) Methane production by ruminants: its contribution to global warming. Ann Zootech 49:231-253.

Nava, D.F. and Stief, L.J. (1989) Temperature study of oxygen atom + phosphine reaction rate: kinetic measurements and planetary atmospheric implications. J Phys Chem 93:4044-4047.

Niu, X., Wei, A., Li, Y., Mi, L., Yang, Z., and Song, X. (2013) Phosphine in paddy fields and the effects of environmental factors. Chemosphere 93:1942-1947.

Noll, K.S. and Marley, M.S. (1997) Detectability of CO, PH3, $\mathrm{AsH} 3$, and $\mathrm{GeH} 4$ in the atmosphere of GL 229B. In: ASP Conference Series Planets Beyond the Solar System and the Next Generation of Space Missions, Baltimore, MD.

Noy, N., Podolak, M., and Bar-Nun, A. (1981) Photochemistry of phosphine and Jupiter's Great Red Spot. J Geophys Res Oceans 86:11985-11988. 
Pascale, E., Bezawada, N., Barstow, J., Beaulieu, J.-P., Bowles, N., du Foresto, V.C., Coustenis, A., Decin, L., Drossart, P., and Eccleston, P. (2018) The ARIEL Space Mission. Space Telescopes and Instrumentation 2018: Optical, Infrared, and Millimeter Wave. International Society for Optics and Photonics, Bellingham, WA.

Pasek, M.A. (2008) Rethinking early Earth phosphorus geochemistry. Proc Natl Acad Sci U S A 105:853-858.

Pasek, M. and Block, K. (2009) Lightning-induced reduction of phosphorus oxidation state. Nat Geosci 2:553.

Pasek, M.A., Mousis, O., and Lunine, J.I. (2011) Phosphorus chemistry on Titan. Icarus 212:751-761.

Pasek, M.A., Harnmeijer, J.P., Buick, R., Gull, M., and Atlas, Z. (2013) Evidence for reactive reduced phosphorus species in the early Archean ocean. Proc Natl Acad Sci U S A 110: 10089-10094.

Pasek, M.A., Sampson, J.M., and Atlas, Z. (2014) Redox chemistry in the phosphorus biogeochemical cycle. Proc Natl Acad Sci U S A 111:15468-15473.

Pech, H., Vazquez, M.G., Van Buren, J., Foster, K.L., Shi, L., Salmassi, T.M., Ivey, M.M., and Pasek, M.A. (2011) Elucidating the redox cycle of environmental phosphorus using ion chromatography. J Chromatogr Sci 49:573-581.

Perkins, M.W., Wong, B., Olivera, D., and Sciuto, A. (2015) Phosphine. In: Hamilton \& Hardy's Industrial Toxicology, edited by M.W. Perkins, B. Wong, D. Olivera, and A. Sciuto, Wiley Online Library, pp 1295-1300, Chapter 118. Available online at https://onlinelibrary.wiley.com/doi/abs/10.1002/9781 118834015.ch118

Petkowski, J.J., Bains, W., and Seager, S. (2019) An apparent binary choice in biochemistry: mutual reactivity implies life chooses thiols or nitrogen-sulfur bonds, but not both. Astrobiology 19:579-613.

Peucker-Ehrenbrink, B. (1996) Accretion of extraterrestrial matter during the last 80 million years and its effect on the marine osmium isotope record. Geochim Cosmochim Acta 60:3187-3196.

Pierrehumbert, R.T. (2010) Principles of Planetary Climate. Cambridge University Press, Cambridge.

Pilcher, C.B. (2003) Biosignatures of early Earths. Astrobiology 3:471-486.

Prinn, R.G. and Lewis, J.S. (1975) Phosphine on Jupiter and implications for the Great Red Spot. Science 190:274-276.

Quanz, S.P., Olivier, A., Angerhausen, S., Benz, W., Bonfils, X., Berger, J.-P., Brogi, M., Cabrera, J., Danchi, W.C., Defrère, D., van Dishoeck, E., Ehrenreich, D., Ertel, S., Fortney, J., Gaudi, S., Girard, J., Glauser, A., Grenfell, L., Ireland, M., Janson, M., Kammerer, J., Kitzmann, D., Kraus, S., Krause, O., Labadie, L., Lacour, S., Lichtenberg, T., Line, M., Linz, H., Loicq, J., Mennesson, B., Meyer, M., Miguel, Y., Monnier, J., N'Diaye, M., Pallé, E., Queloz, D., Rauer, H., Ribas, I., Rugheimer, S., Selsis, F., Serabyn, G., Snellen, I., Sozzetti, A., Stapelfeldt, K., Triaud, A., Udry, S., and Wyatt, M. (2019) Atmospheric characterization of terrestrial exoplanets in the mid-infrared: biosignatures, habitability \& diversity. arXiv Preprint arXiv:1908.01316.

Ranjan, S. and Sasselov, D.D. (2017) Constraints on the early terrestrial surface UV environment relevant to prebiotic chemistry. Astrobiology 17:169-204.

Roberge, A. (2019) The Large UV/Optical/Infrared Surveyor (LUVOIR): Telling the Story of Life in the Universe. Astro2020 APC White Paper.

Roels, J. and Verstraete, W. (2001) Biological formation of volatile phosphorus compounds. Bioresour Technol 79:243-250.
Roels, J. and Verstraete, W. (2004) Occurrence and origin of phosphine in landfill gas. Sci Total Environ 327:185-196.

Roels, J., Huyghe, G., and Verstraete, W. (2005) Microbially mediated phosphine emission. Sci Total Environ 338:253-265.

Rothman, L.S., Gordon, I.E., Barber, R.J., Dothe, H., Gamache, R.R., Goldman, A., Perevalov, V.I., Tashkun, S.A., and Tennyson, J. (2010) HITEMP, the high-temperature molecular spectroscopic database. J Quant Spectrosc Radiat Transfer 111:2139-2150.

Rugheimer, S., Kaltenegger, L., Segura, A., Linsky, J., and Mohanty, S. (2015) Effect of UV radiation on the spectral fingerprints of earth-like planets orbiting M stars. Astrophys $J$ 809:57.

Rutishauser, B.V. and Bachofen, R. (1999) Phosphine formation from sewage sludge cultures. Anaerobe 5:525-531.

Sander, R. (2015) Compilation of Henry's law constants (version 4.0) for water as solvent. Atmos Chem Phys 15:4399-4981.

Sander, R., Baumgaertner, A., Gromov, S., Harder, H., Jöckel, P., Kerkweg, A., Kubistin, D., Regelin, E., Riede, H., and Sandu, A. (2011) The atmospheric chemistry box model CAABA/MECCA-3.0. Geosci Model Dev 4:373-380

Schink, B. and Friedrich, M. (2000) Bacterial metabolism: phosphite oxidation by sulphate reduction. Nature 406:37-37.

Schwieterman, E.W., Kiang, N.Y., Parenteau, M.N., Harman, C.E., DasSarma, S., Fisher, T.M., Arney, G.N., Hartnett, H.E., Reinhard, C.T., Olson, S.L. Meadows, V.S., Cockell, C.S., Walker, S., Grenfell, J.L., Hegde, S., Rugheimer, S., $\mathrm{Hu}, \mathrm{R}$., and Lyons, T.W. (2018) Exoplanet biosignatures: a review of remotely detectable signs of life. Astrobiology 18: 663-708.

Seager, S. (2010) Exoplanet Atmospheres: Physical Processes. Princeton University Press, Princeton, NJ.

Seager, S., Bains, W., and Hu, R. (2013a) A biomass-based model to estimate the plausibility of exoplanet biosignature gases. Astrophys J 775:104.

Seager, S., Bains, W., and Hu, R. (2013b) Biosignature gases in H2-dominated atmospheres on rocky exoplanets. Astrophys $J$ 777:95.

Seager, S., Bains, W., and Petkowski, J.J. (2016) Toward a list of molecules as potential biosignature gases for the search for life on exoplanets and applications to terrestrial biochemistry. Astrobiology 16:465-485.

Segura, A., Kasting, J.F., Meadows, V., Cohen, M., Scalo, J., Crisp, D., Butler, R.A., and Tinetti, G. (2005) Biosignatures from Earth-like planets around $\mathrm{M}$ dwarfs. Astrobiology 5: 706-725.

Skidmore, W., Teams, T.M.T.I.S.D., and Committee, T.M.T.S.A. (2015) Thirty meter telescope detailed science case: 2015. Res Astron Astrophys 15:1945.

Sousa-Silva, C., Yurchenko, S.N., and Tennyson, J. (2013) A computed room temperature line list for phosphine. $J \mathrm{Mol}$ Spectrosc 288:28-37.

Sousa-Silva, C., Hesketh, N., Yurchenko, S.N., Hill, C., and Tennyson, J. (2014) High temperature partition functions and thermodynamic data for ammonia and phosphine. J Quant Spectrosc Radiat Transfer 142:66-74.

Sousa-Silva, C., Al-Refaie, A.F., Tennyson, J., and Yurchenko, S.N. (2015) ExoMol line lists-VII. The rotation-vibration spectrum of phosphine up to 1500 K. Mon Not R Astron Soc 446:2337-2347.

Sousa-Silva, C., Petkowski, J.J., and Seager, S. (2018) ATMOS: towards Resolving Ambiguities in the Spectroscopic Detection of Life. Astronomical Society of the Pacific Conference Series, San Francisco, p 241. 
Sousa-Silva, C., Petkowski, J.J., and Seager, S. (2019) Molecular simulations for the spectroscopic detection of atmospheric gases. Phys Chem Chem Phys 21:18970-18987.

Tamai, R. and Spyromilio, J. (2014) European Extremely Large Telescope: Progress Report. SPIE Astronomical Telescopes+Instrumentation, Bellingham, WA.

Tarrago, G., Lacome, N., Lévy, A., Guelachvili, G., Bézard, B., and Drossart, P. (1992) Phosphine spectrum at 4-5 $\mu \mathrm{m}$ : analysis and line-by-line simulation of $2 v 2, v 2+v 4,2 v 4, v 1$, and $v 3$ bands. $J$ Mol Spectrosc 154:30-42.

Teanby, N.A., Irwin, P.G.J., and Moses, J.I. (2019) Neptune's carbon monoxide profile and phosphine upper limits from Herschel/SPIRE: implications for interior structure and formation. Icarus 319:86-98.

Tennyson, J., Yurchenko, S.N., Al-Refaie, A.F., Barton, E.J., Chubb, K.L., Coles, P.A., Diamantopoulou, S., Gorman, M.N., Hill, C., Lam, A.Z., Lodi, L., McKemmish, L.K., Na, Y., Owens, A., Polyansky, O.L., Sousa-Silva, C., Underwood, D.S., Yachmenev, A., and Zak, E. (2016) The ExoMol database: molecular line lists for exoplanet and other hot atmospheres. J Mol Spectrosc 327:73-94.

Tessenyi, M., Tinetti, G., Savini, G., and Pascale, E. (2013) Molecular detectability in exoplanetary emission spectra. Icarus 226:1654-1672.

Visconti, G. (1981) Multiple scattering and photochemistry in planetary atmospheres. Memorie della Societa Astronomica Italiana [Journal of the Italian Astronomical Society]. 52:535.

Visscher, C., Lodders, K., and Jr. B. F. (2006) Atmospheric chemistry in giant planets, brown dwarfs, and low-mass dwarf stars. II. Sulfur and phosphorus. Astrophys J 648: 1181 .

Winters, J.G., Henry, T.J., Jao, W.-C., Subasavage, J.P., Chatelain, J.P., Slatten, K., Riedel, A.R., Silverstein, M.L., and Payne, M.J. (2019) The solar neighborhood. XLV. The stellar multiplicity rate of M dwarfs within 25 pc. Astron J 157:216.

Wolf, E.T., and Toon, O.B. (2010) Fractal organic hazes provided an ultraviolet shield for early Earth. Science 328:12661268.

Wright, G.S., Rieke, G., Boeker, T., Colina, L., Van Dishoeck, E., Driggers, P., Friedman, S., Glasse, A., Goodson, G., and Greene, T. (2010) Progress with the Design and Development of MIRI, the Mid-IR Instrument for JWST. International Society for Optics and Photonics, Bellingham, WA.

Yaroshevsky, A.A. (2006) Abundances of chemical elements in the Earth's crust. Geochem Int 44:48-55.

Yu, X., Geng, J., Ren, H., Chao, H., and Qiu, H. (2015) Determination of phosphite in a full-scale municipal wastewater treatment plant. Environ Sci Processes Impacts 17:441-447.

Yurchenko, S.N. and Tennyson, J. (2014) ExoMol line listsIV. The rotation-vibration spectrum of methane up to 1500 K. Mon Not R Astron Soc 440:1649-1661.

Yurchenko, S.N., Barber, R.J., and Tennyson, J. (2011) A variationally computed line list for hot NH3. Mon Not $R$ Astron Soc 413:1828-1834.

Yurchenko, S.N., Al-Refaie, A.F., and Tennyson, J. (2018) ExoCross: a general program for generating spectra from molecular line lists. Astron Astrophys 614:A131.
Zahnle, K.J. (1986) Photochemistry of methane and the formation of hydrocyanic acid (HCN) in the Earth's early atmosphere. J Geophys Res Atmos 91:2819-2834.

Zahnle, K.J., Gacesa, M., and Catling, D.C. (2019) Strange messenger: a new history of hydrogen on Earth, as told by Xenon. Geochim Cosmochim Acta 244:56-85.

Zhang, R., Wu, M., Wang, Q., Geng, J., and Yang, X. (2010) The determination of atmospheric phosphine in Ny-Ålesund. Chinese Sci Bull 55:1662-1666.

Zhu, R., Kong, D., Sun, L., Geng, J., Wang, X., and Glindemann, D. (2006a) Tropospheric phosphine and its sources in coastal Antarctica. Environ Sci Technol 40:7656-7661.

Zhu, R., Sun, L., Kong, D., Geng, J., Wang, N., Wang, Q., and Wang, X. (2006b) Matrix-bound phosphine in Antarctic biosphere. Chemosphere 64:1429-1435.

Zhu, R., Glindemann, D., Kong, D., Sun, L., Geng, J., and Wang, X. (2007a) Phosphine in the marine atmosphere along a hemispheric course from China to Antarctica. Atmos Environ 41:1567-1573.

Zhu, R., Kong, D., Sun, L., Geng, J., and Wang, X. (2007b) The first determination of atmospheric phosphine in Antarctica. Chinese Sci Bull 52:131-135.

Zhu, R., Wang, Q., Ding, W., Wang, C., Hou, L., and Ma, D. (2014) Penguins significantly increased phosphine formation and phosphorus contribution in maritime Antarctic soils. Sci Rep 4:7055.

Address correspondence to:

Clara Sousa-Silva

Department of Earth, Atmospheric, and Planetary Sciences Massachusetts Institute of Technology (MIT)

77 Massachusetts Avenue

Building 54, 54-1719

Cambridge, MA 02139

E-mail: clara@space.mit.edu

Submitted 10 September 2018 Accepted 7 October 2019

Abbreviations Used
ARIEL $=$ Atmospheric Remote-sensing Infrared
$\quad$ Exoplanet Large-survey
HabEx $=$ Habitable Exoplanet Imaging Mission
$\mathrm{JWST}=$ James Webb Space Telescope
$\mathrm{LUVIR}=$ Large UV Optical Infrared Surveyor
$\mathrm{MBP}=$ Matrix-Bound Phosphine
$\mathrm{NUV}=$ Near UV
$\mathrm{OST}=$ Origins Space Telescope
$\mathrm{PH} \mathrm{H}_{3}=$ phosphine
$\mathrm{SEAS}=$ Simulated Exoplanet Atmosphere
Spectra
$\mathrm{UV}=$ ultraviolet




\section{Appendix}

\section{Appendix A: Atmospheric Mixing Ratios and Temperature-Pressure Profiles}

Appendix Figure A1 shows the molecular mixing ratio profiles used to simulate atmospheric spectra for the planets with $\mathrm{CO}_{2^{-}}$and $\mathrm{H}_{2}$-rich atmospheres orbiting active $\mathrm{M}$ dwarfs, quiet $\mathrm{M}$ dwarfs, and Sun-like stars. To establish the effect of adding phosphine to the atmosphere, we ran the photochemical models with the starting mixing ratios from Appendix Fig. A1 considering surface fluxes of phosphine $\left(\mathrm{PH}_{3}\right)$ ranging from $10^{3} \mathrm{~cm}^{-2} \mathrm{~s}^{-1}$ to $10^{14} \mathrm{~cm}^{-2} \mathrm{~s}^{-1}$.

Appendix Figure A2 shows the temperature-pressure profiles used to simulate the transmission and emission spectra for the planets with $\mathrm{H}_{2^{-}}$and $\mathrm{CO}_{2}$-rich atmospheres.
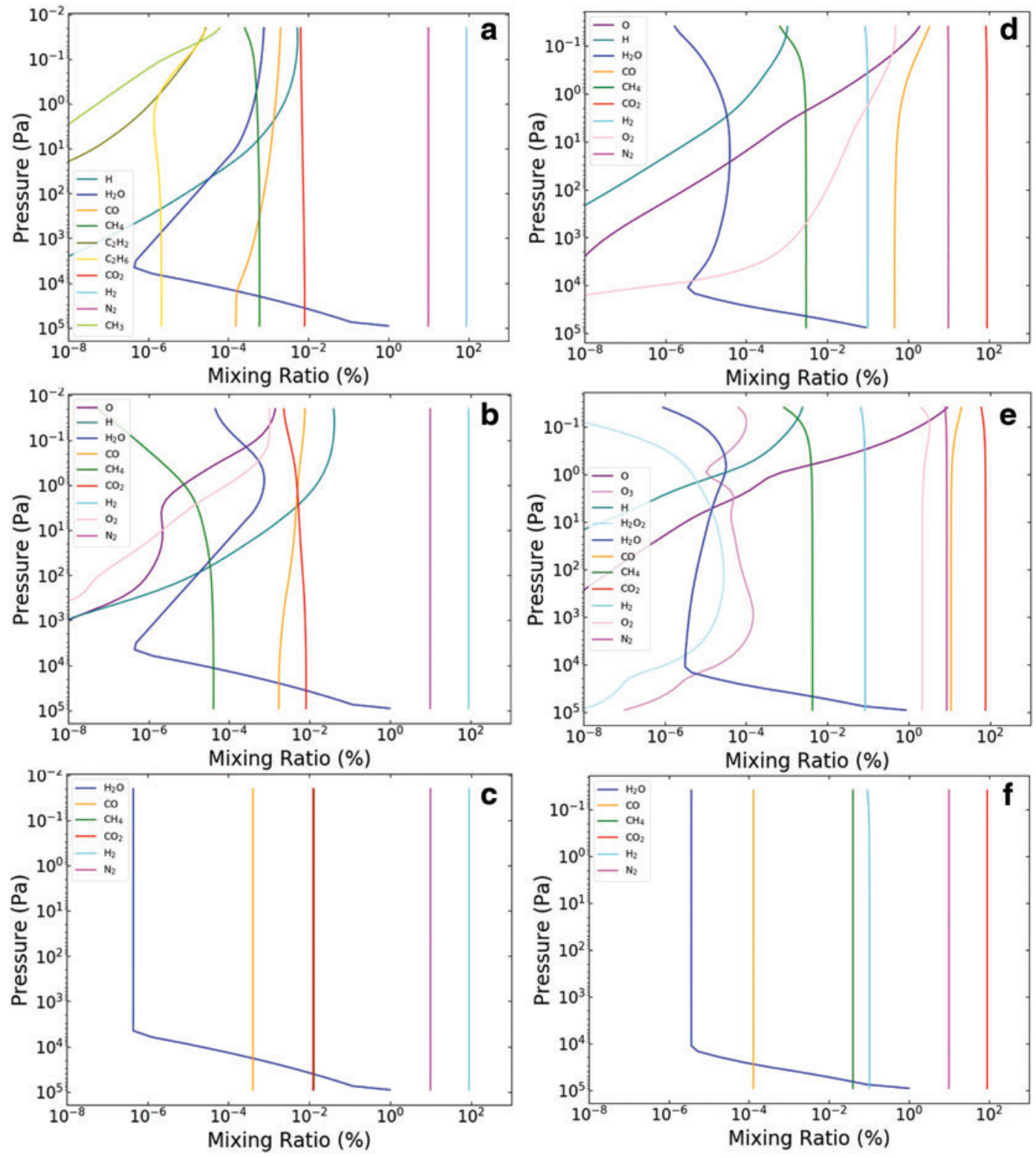

APPENDIX FIG. A1. Mixing ratio profiles of simulated Earth-sized planets with the following parameters: (a) $\mathrm{H}_{2-}$ dominated atmosphere on a planet orbiting a Sun-like star; (b) $\mathrm{H}_{2}$-rich atmosphere on a planet orbiting an active $\mathrm{M}$ dwarf; (c) $\mathrm{H}_{2}$-rich atmosphere on a planet orbiting a quiet $\mathrm{M}$ dwarf; (d) $\mathrm{CO}_{2}$-rich atmosphere on a planet orbiting a Sun-like star; (e) $\mathrm{CO}_{2}$-rich atmosphere on a planet orbiting an active $\mathrm{M}$ dwarf; (f) $\mathrm{CO}_{2}$-rich atmosphere on a planet orbiting a quiet $\mathrm{M}$ dwarf. Vertical axis represents pressure in units of $\mathrm{Pa}$, and horizontal axis shows the mixing ratio represented as a percentage of the total atmosphere. Figure partially adapted from the works of $\mathrm{Hu}$ et al. (2012) and Seager et al. (2013). 


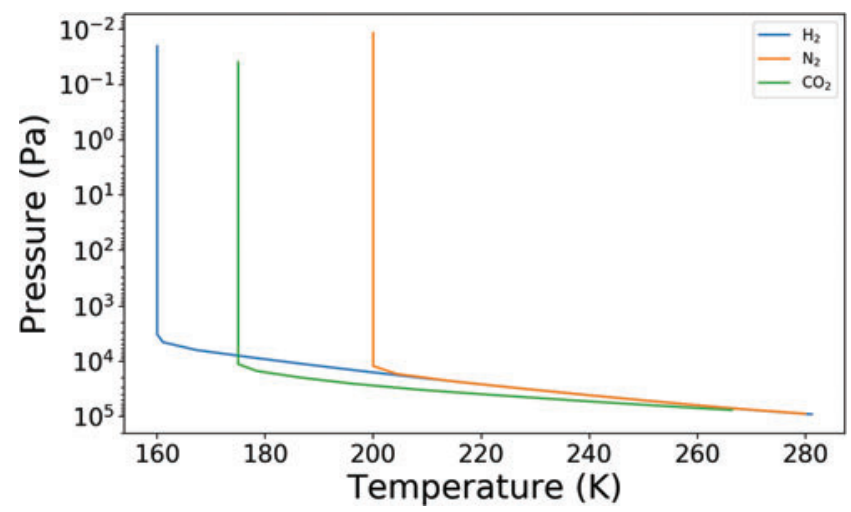

APPENDIX FIG. A2. Temperature-pressure profiles of the simulated massive super-Earths $\left(M_{p}=10 M_{E}\right.$ and $R_{p}=1.75$ $\left.R_{E}\right)$, with $\mathrm{H}_{2-}$ and $\mathrm{CO}_{2}$-rich atmospheres $\left(\mathrm{N}_{2}\right.$-rich atmosphere shown for comparison as an intermediate reducing scenario). Vertical axis represents pressure in units of $\mathrm{Pa}$, and horizontal axis shows the temperature in units of $\mathrm{K}$. Figure adapted from the work of Hu et al. (2012).

\section{Appendix B: Detailed Spectral Comparison of Phosphine with Other Atmospheric Components}

The three strongest spectral features of phosphine occur at 2.7-3.6, 4.0-4.8, and 7.8-11.5 $\mu \mathrm{m}$. Appendix Figures B1 and B2 (2-6.5 and $7.5-11.8 \mu \mathrm{m}$, respectively) show a detailed comparison between the room temperature cross sections of $\mathrm{PH}_{3}$ and six common atmospheric molecules, namely $\mathrm{H}_{2} \mathrm{O}, \mathrm{CH}_{4}, \mathrm{CO}_{2}, \mathrm{CO}, \mathrm{NH}_{3}$, and $\mathrm{H}_{2} \mathrm{~S}$.

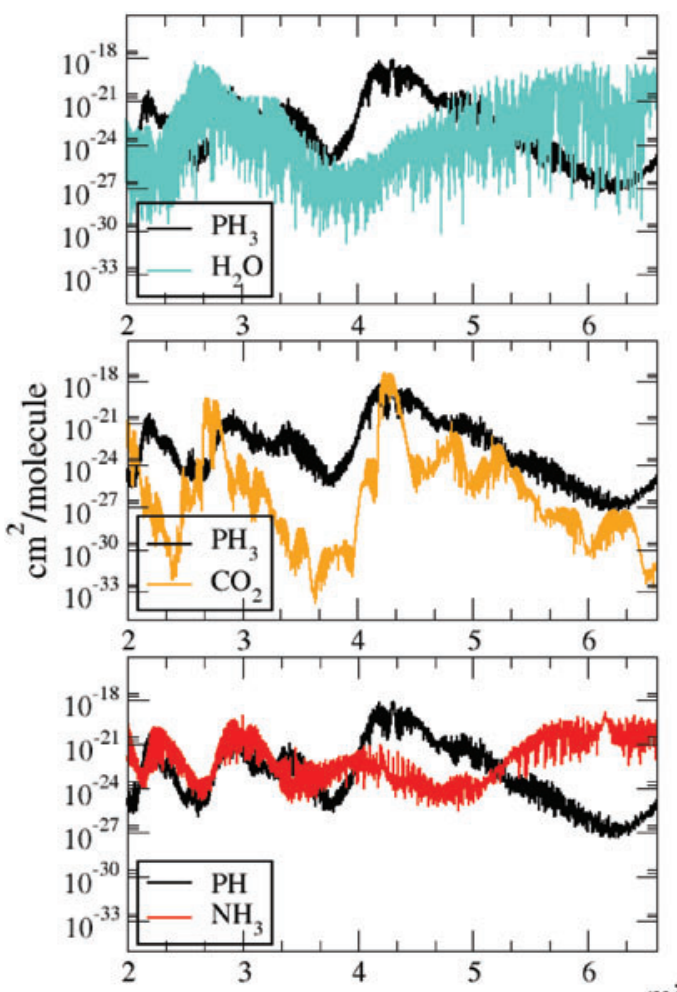

\section{Appendix C: Thermodynamics of Abiotic Phosphine Synthesis at Circumneutral pH}

We conduct a thermodynamic analysis to determine whether the formation of phosphine from phosphate and phosphite is thermodynamically favored at circumneutral $\mathrm{pH}$. We calculate the free energy of formation of $\mathrm{PH}_{3}$ in solution (and solid, in reduction of calcium phosphate) under geochemical source conditions and elemental composition similar to
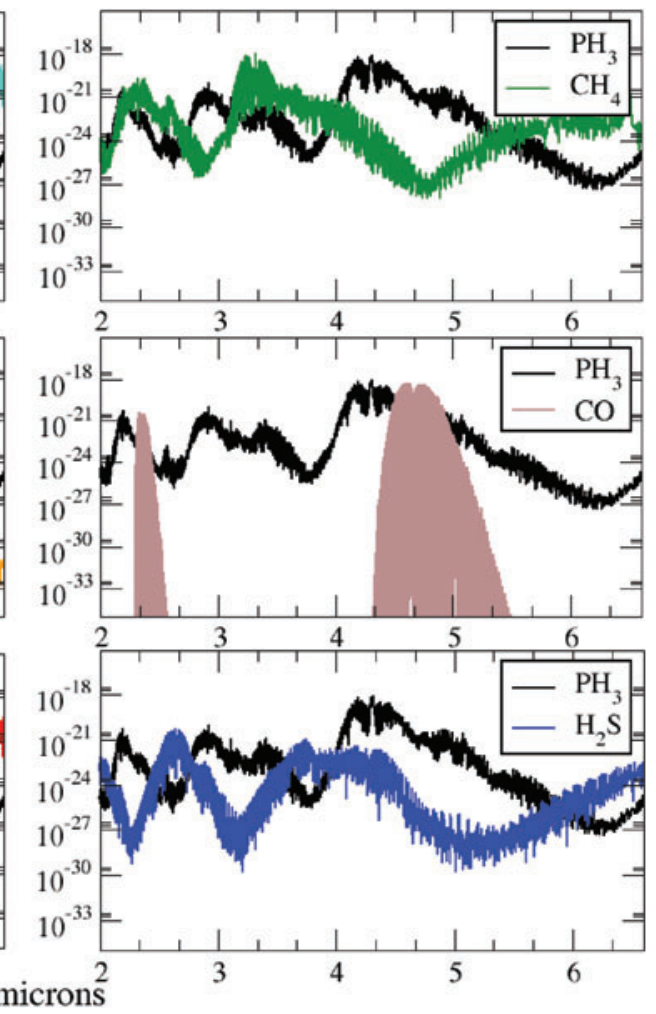

APPENDIX FIG. B1. Comparison of the spectral cross sections of phosphine with other gases at room temperature, in the $2-6.5 \mu \mathrm{m}$ region. Intensity on $y$-axes with units of $\mathrm{cm}^{2} /$ molecule and wavelength represented on the $x$-axes in microns. All cross sections are calculated at zero-pressure (i.e., Doppler-broadened lines only) using the procedure described by Hill et al. (2013). $\mathrm{PH}_{3}$, shown in black, is distinguishable in this region from all compared molecules. 

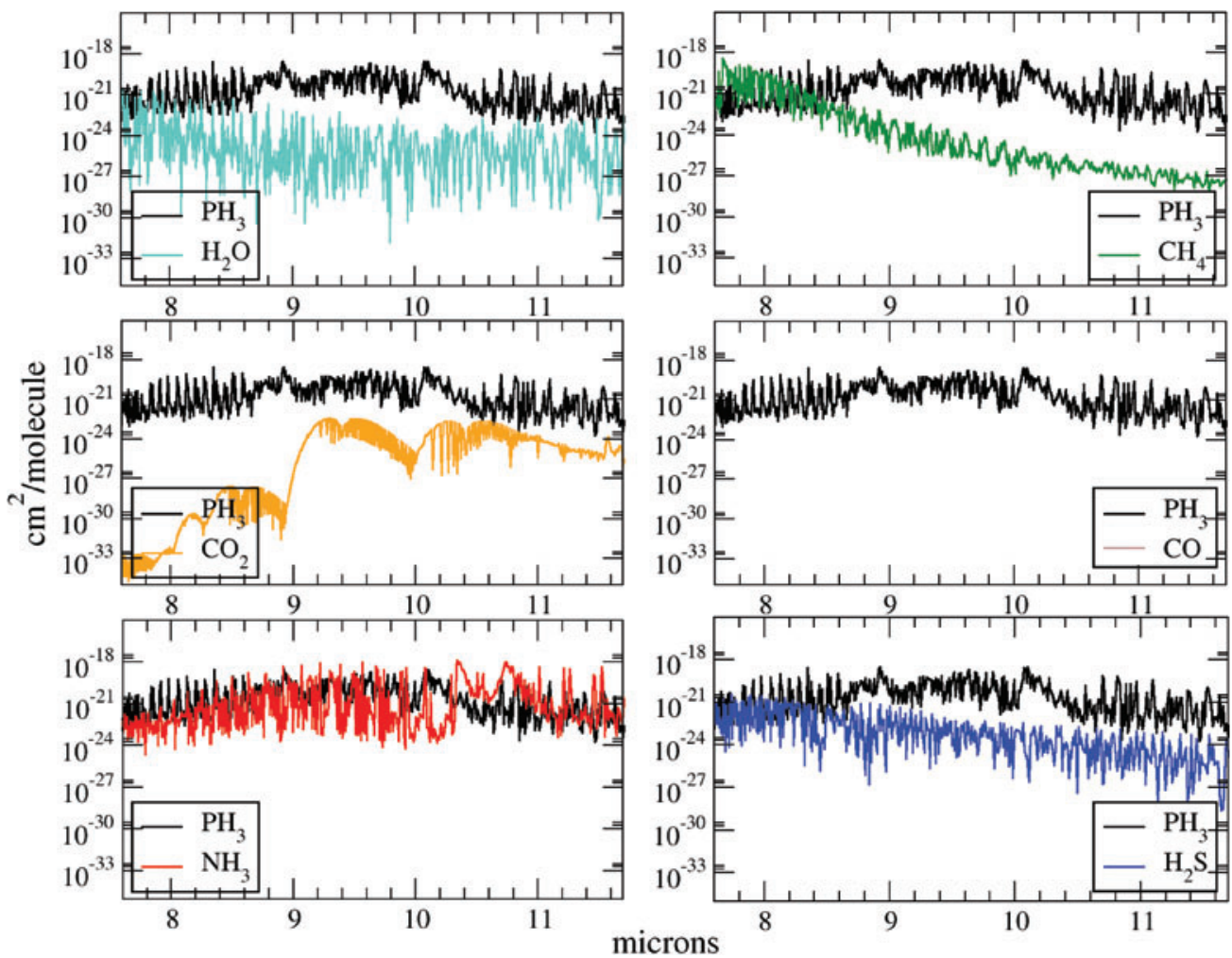

APPENDIX FIG. B2. Comparison of the spectral cross sections of phosphine with other gases at room temperature, in the $7.6-11.7 \mu \mathrm{m}$ region. Intensity on $y$-axes with units of $\mathrm{cm}^{2} /$ molecule and wavelength represented on the $x$-axes in microns. All cross sections are calculated at zero-pressure (i.e., Doppler-broadened lines only) using the procedure described by Hill et al. (2013). $\mathrm{PH}_{3}$, shown in black, is distinguishable in this region from all compared molecules except ammonia. Note that $\mathrm{CO}$ is not known to absorb in this region.

terrestrial planets for two temperature conditions $\left(0^{\circ} \mathrm{C}\right.$ and $140^{\circ} \mathrm{C}$, or 273 and $413 \mathrm{~K}$ ) and two extreme $\mathrm{H}_{2}$ abundance levels (Appendix Table C1) on the basis of established thermodynamic values (Barner and Scheurman, 1978; Conrad et al., 1986; Amend and Shock, 2001; Linstrom and Mallard, 2001; Fu et al., 2013). The free energy of reaction of formation of $\mathrm{PH}_{3}$ is given by:

$$
\Delta G=\Delta G^{\mathrm{o}}+R \cdot T \cdot \ln (Q)
$$

where $\Delta G^{\mathrm{o}}$ is the standard free energy of formation, $R$ is the gas constant, $T$ is the absolute temperature, and $Q$ is the reaction quotient.

The resulting calculated free energy of formation of phosphine can be used as a proxy to estimate the likelihood of $\mathrm{PH}_{3}$ formation as a geochemical false positive (Bains et al., 2017). The more positive the free energy of formation of $\mathrm{PH}_{3}$ for a given reaction pathway, the less likely it is to be produced through geochemical processes. We note that the estimation of likelihood of geochemical false-positive scenarios for $\mathrm{PH}_{3}$ is a part of the larger effort to estimate the possibilities for false positives for all biosignature gases (Bains et al., 2017).

The calculated energy of formation of phosphine is strongly positive for any of the proposed $\mathrm{PH}_{3}$ formation reaction pathways, given the geochemical concentrations of gases, suggesting that if $\mathrm{PH}_{3}$ is detected in an atmosphere of an exoplanet, its source is not likely to be geochemical, increasing the probability for biological production (Appendix Table $\mathrm{C} 1$ ).

\section{Appendix D: Production of Phosphine from Phosphite at Acidic $\mathrm{pH}$}

It has been suggested that abiotic disproportionation of phosphites could be a source of environmental phosphine (Roels and Verstraete, 2001). Indeed, one common laboratory method of obtaining $\mathrm{PH}_{3}$ is by heating phosphite, which disproportionates to phosphine and phosphate (Gokhale et al., 2007). This process is only favored at high temperature $\left(T>50^{\circ} \mathrm{C}\right.$ or $T>323 \mathrm{~K}$ ) or acid conditions ( $\mathrm{pH} \sim<1.3$, where $\mathrm{H}_{3} \mathrm{PO}_{3}$ and/or $\mathrm{H}_{2} \mathrm{PO}_{3}{ }^{-}$dominate) (Appendix Fig. D1). We also note that the production of phosphite via the reduction of phosphate with $\mathrm{H}_{2}$ on Earth-analog habitable worlds is highly endergonic and is also unlikely (Appendix Table D1). For a detailed analysis of thermodynamic limitations of $\mathrm{PH}_{3}$ and phosphite production, see Bains et al. (2019a).

Such acidic and high-temperature conditions are not characteristic of Earth-analog habitable worlds, although they are available at "black smoker" vents (Martin et al., 2008), and might be more common on a hot world with more acid oceans, for example, a world with high $\mathrm{pCO}_{2}$ orbiting close to its parent star. We conclude that disproportionation of phosphite is unlikely to generate significant fluxes of phosphines on Earth-analog habitable worlds, but that worlds which are known to have hot extremely acid 
Appendix Table C1. Reaction Pathways and the Energy of Formation of Phosphine from Plausible Geochemical Volatile Concentrations

(1) Oxidized atmosphere (low $\mathrm{H}_{2}$ levels)

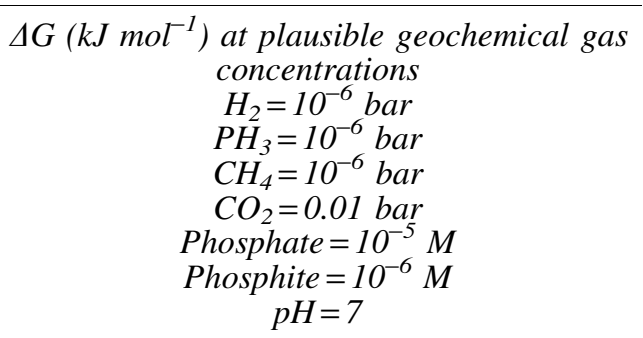

\begin{tabular}{|c|c|c|c|}
\hline Possible geochemical $\mathrm{PH}_{3}$ formation reaction & $\Delta G^{o}\left(k J \mathrm{~mol}^{-1}\right)$ & Temp. $=0^{\circ} \mathrm{C}$ & Temp. $=140^{\circ} \mathrm{C}$ \\
\hline $\mathrm{HPO}_{4}{ }^{2-}(\mathrm{ag})+4 \mathrm{H}_{2(\mathrm{ag})} \rightarrow \mathrm{PH}_{3(\mathrm{ag})}+2 \mathrm{H}_{2} \mathrm{O}_{(\mathrm{g})}+2 \mathrm{OH}_{(\mathrm{ag})}^{-}$ & 225.16 & 410.14 & 538.49 \\
\hline $\mathrm{HPO}_{4}{ }^{2}$ (aq) $+\mathrm{CH}_{4(\mathrm{aq})} \rightarrow \mathrm{PH}_{3(\mathrm{aq})}+\mathrm{CO}_{2(\mathrm{aq})}+2 \mathrm{OH}^{-}(\mathrm{aq})$ & 415.32 & 503.97 & 558.16 \\
\hline $\mathrm{HPO}_{3}{ }_{(\mathrm{aq})}+3 \mathrm{H}_{2(\mathrm{aq})} \rightarrow \mathrm{PH}_{3(\mathrm{aq})}+\mathrm{H}_{2} \mathrm{O}_{(\mathrm{l})}+2 \mathrm{OH}_{(\mathrm{aq})}^{-}$ & 130.79 & 303.69 & 355.06 \\
\hline $\begin{array}{l}\mathrm{HPO}_{3}{ }^{2-(a q)}+1 / 2 \mathrm{H}_{2} \mathrm{O}_{(1)}+3 / 4 \mathrm{CH}_{4(\mathrm{aq})} \rightarrow \mathrm{PH}_{3(\mathrm{aq})} \\
\quad+3 / 4 \mathrm{CO}_{2(\mathrm{aq})}+2 \mathrm{OH}^{-}(\mathrm{aq})\end{array}$ & 358.80 & 451.88 & 459.38 \\
\hline $\begin{array}{l}1 / 2 \mathrm{Ca}_{3}\left(\mathrm{PO}_{4}\right)_{2(\mathrm{~s})}+4 \mathrm{H}_{2(\mathrm{~g})} \rightarrow \mathrm{PH}_{3(\mathrm{~g})}+1 \frac{1 / 2 \mathrm{Ca}(\mathrm{OH})_{2(\mathrm{~s})}}{}+\mathrm{H}_{2} \mathrm{O}_{(\mathrm{g})}\end{array}$ & 373.32 & 465.08 & 526.75 \\
\hline
\end{tabular}

(2) Reduced atmosphere (high $\mathrm{H}_{2}$ levels)

$\Delta G\left(\mathrm{~kJ} \mathrm{~mol}^{-1}\right)$ at plausible geochemical gas
concentrations
$\mathrm{H}_{2}=1 \mathrm{bar}$
$\mathrm{PH}_{3}=10^{-6} \mathrm{bar}$
$\mathrm{CH}_{4}=10^{-1} \mathrm{bar}$
$\mathrm{CO}_{2}=0.01$ bar
Phosphate $=10^{-6} \mathrm{M}$
Phosphite $=10^{-5} \mathrm{M}$
pH $=7$

\begin{tabular}{|c|c|c|c|}
\hline Possible geochemical $\mathrm{PH}_{3}$ formation reaction & $\Delta G^{o}\left(k^{J} \mathrm{~mol}^{-1}\right)$ & Temp. $=0^{\circ} \mathrm{C}$ & Temp. $=140^{\circ} \mathrm{C}$ \\
\hline $\begin{array}{l}\mathrm{HPO}_{4}{ }^{2-}{ }^{2}{ }^{(\mathrm{aq})}+4 \mathrm{H}_{2(\mathrm{aq})} \rightarrow \mathrm{PH}_{3(\mathrm{aq})}+2 \mathrm{H}_{2} \mathrm{O}_{(\mathrm{l})}+2 \mathrm{OH}^{-}(\mathrm{aq}) \\
\mathrm{HPO}_{4}{ }^{-}{ }^{(\mathrm{aq})}+\mathrm{CH}_{4(\mathrm{aq})} \rightarrow \mathrm{PH}_{3(\mathrm{aq})}+\mathrm{CO}_{2(\mathrm{aq})}+2 \mathrm{OH}^{-}{ }_{(\mathrm{aq})} \\
\mathrm{HPO}_{3}{ }^{2-}{ }^{(\mathrm{aq})}+3 \mathrm{H}_{2(\mathrm{aq})} \rightarrow \mathrm{PH}_{3(\mathrm{aq})}+\mathrm{H}_{2} \mathrm{O}_{(\mathrm{l})}+2 \mathrm{OH}^{-}(\mathrm{aq}) \\
\mathrm{HPO}_{3}{ }^{-{ }_{(\mathrm{aq})}}+\mathrm{CH}_{4(\mathrm{aq})} \rightarrow \mathrm{PH}_{3(\mathrm{aq})}+\mathrm{CO}_{(\mathrm{aq})}+2 \mathrm{OH}^{-}{ }_{(\mathrm{aq})} \\
1 / 2 \mathrm{Ca}_{3}\left(\mathrm{PO}_{4}\right)_{2(\mathrm{~s})}+4 \mathrm{H}_{2(\mathrm{~g})} \rightarrow \mathrm{PH}_{3(\mathrm{~g})}+1 \frac{1}{2} \mathrm{Ca}(\mathrm{OH})_{2(\mathrm{~s})}+\mathrm{H}_{2} \mathrm{O}_{(\mathrm{g})}\end{array}$ & $\begin{array}{l}225.16 \\
415.32 \\
130.79 \\
358.80 \\
373.32\end{array}$ & $\begin{array}{l}289.95 \\
483.07 \\
204.40 \\
427.06 \\
339.65\end{array}$ & $\begin{array}{l}356.65 \\
526.54 \\
204.84 \\
421.83 \\
337.00\end{array}$ \\
\hline
\end{tabular}

Free energy of formation of phosphine $(\Delta G)$ was calculated under geochemical concentration of gases for two different temperature scenarios and two $\mathrm{H}_{2}$ levels (1) oxidized atmosphere (low $\mathrm{H}_{2}$ levels) and (2) reduced atmosphere (high $\mathrm{H}_{2}$ levels). Under all the tested terrestrial planet conditions, the free energy of formation of $\mathrm{PH}_{3}(\Delta G)$ is positive, making the geochemical formation of $\mathrm{PH}_{3}$ from phosphate and phosphite an unlikely scenario at circumneutral $\mathrm{pH}$.

oceans $\left(T>\sim 50^{\circ} \mathrm{C} / 323 \mathrm{~K}, \mathrm{pH}<\sim 1.3\right)$ may be vulnerable to this false-positive scenario.

\section{Appendix E: Phosphine in the Context of Terrestrial Biology}

We briefly address a few of the common concerns in the association of phosphine with terrestrial biology: the toxicity of $\mathrm{PH}_{3}$; the absence of a known metabolic biosynthetic pathway and enzymatic mechanism for the production of $\mathrm{PH}_{3}$; some context on the ecology of phosphines; and a discussion of the phosphorus cycle.

\section{Phosphine Toxicity}

On modern Earth, phosphine is a rare toxic gas. It is highly toxic to aerobically metabolizing organisms (Bond and Monro, 1967), which is reflected by its wide use as fumigant [e.g., reviewed in Perkins et al. (2015)]. The toxicity of $\mathrm{PH}_{3}$ appears to be strictly dependent on aerobic metabolism [e.g., reviewed in Valmas et al. (2008)]. The detailed analysis of the toxic effects of $\mathrm{PH}_{3}$ on aerobic organisms is beyond the scope of this article and is published elsewhere (Bains et al., 2019b). However, if $\mathrm{PH}_{3}$ chemistry is indeed selectively incompatible with $\mathrm{O}_{2}$-dependent metabolism of aerobic organisms, as literature suggests, it opens an intriguing possibility that phosphines may safely be produced as secondary metabolites to much greater extent by obligatorily anaerobic life, which does not rely on $\mathrm{O}_{2}$ metabolism (Bains et al., 2019b).

We note that it is not known why phosphine is so much more toxic to oxygenic organisms than anoxic ones, given that both rely on heme iron atoms that interact with $\mathrm{PH}_{3}$ (Bains et al., 2019b). We postulate that the highly positive electrode potential of the oxygen-handling enzymes may 


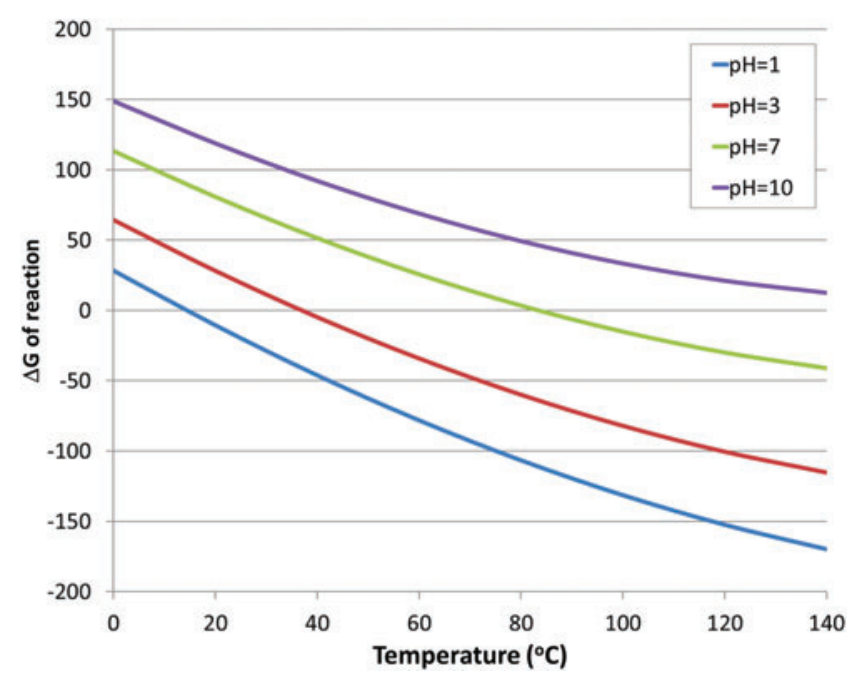

APPENDIX FIG. D1. Phosphine production by disproportionation of phosphite is favored under very low $\mathrm{pH}$ or high temperature conditions. $Y$ axis: $\Delta G^{\mathrm{o}}$ (Gibbs free energy of reaction under standard conditions), in units of $\mathrm{kJ} \mathrm{mol}^{-1}$. $X$ axis: temperature $\left({ }^{\circ} \mathrm{C}\right)$. The free energy of reaction for four reactions are shown: disproportionation at very low $\mathrm{pH}$, where phosphite is present as unionized $\mathrm{H}_{3} \mathrm{PO}_{3}$ form; and disproportionation of ionized species present at higher $\mathrm{pHs}$ (see Bains et al., 2019a for more details on calculation method).

make them more susceptible targets for $\mathrm{PH}_{3}$ complexation and hence inactivation.

\section{Biosynthetic Pathways of Phosphine and Other Volatiles}

The identification of natural products from anaerobic organisms and elucidation of their biosynthetic pathways is notoriously difficult. Culturing of anaerobic organisms is much more laborious task than aerobic ones, and identification or purification of anaerobic metabolites is much more complex as well. It requires specialized experimental setup, as often isolated molecules or their biosynthetic precursors get destroyed in our oxygen-rich atmosphere before they can be properly studied. It is therefore not surprising that the exact molecular mechanism of biological phosphine formation in anaerobic environment has eluded discovery for such a long time. Even identification of biosynthetic pathways of natural molecules from aerobic organisms that can be easily cultured in the laboratory conditions can take many decades. For example, the biosynthetic pathway of

\begin{tabular}{|c|c|c|}
\hline \multicolumn{3}{|c|}{ 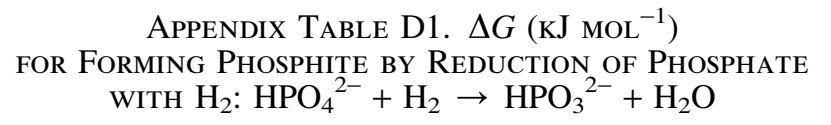 } \\
\hline & $\begin{array}{r}\Delta \mathrm{G}(\mathrm{kJ} \text { mol } \\
\text { oxidizing cond } \\
\text { (as above in } A p\end{array}$ & $\begin{array}{l}\Delta \mathrm{G}\left(\mathrm{kJ} \mathrm{mol}^{-1}\right) \text {; } \\
\text { reducing conditions } \\
\text { (as above in Appendix }\end{array}$ \\
\hline Temperature & Table C1 & Table C1) \\
\hline $0^{\circ} \mathrm{C} / 273 \mathrm{~K}$ & 38.52 & 17.62 \\
\hline $140^{\circ} \mathrm{C} / 413 \mathrm{~K}$ & 95.36 & 63.73 \\
\hline
\end{tabular}

nucleocidin, an unusual fluorine-containing natural antibiotic produced by a bacterium Streptomyces calvus, was only recently elucidated, after $\sim 60$ years of intensive studies (Petkowski et al., 2018).

The economically important production of volatile molecules by fruiting bodies of truffles (Tuber sp.), responsible for the unique aroma of the fungus, is another good illustration of the complexity of the biological production of volatile and nonvolatile natural metabolites. Despite many decades of studies, and an influx of funding from a multimillion-dollar food industry, the biosynthetic pathways responsible for synthesis of truffle volatiles are not fully understood (Zambonelli et al., 2016). The biological production of truffle volatiles, for example, thiophene derivatives, is further complicated by the fact that the biosynthetic pathways for molecules responsible for the unique aroma of truffles are likely shared between multiple species of symbiotic microorganisms (bacteria and yeast) living within the fruiting body of the fungus. The metabolic processes of the symbionts and the fungus host collectively allow for the formation of the final volatile product (e.g., thiophene derivatives) (Zambonelli et al., 2016). Each of the symbionts, and the host fungus, likely contain only part of the full biosynthetic pathway required for the formation of the final volatile product, and each single species is only responsible for the production of few biosynthetic intermediates for the formation of thiophene derivatives (Zambonelli et al., 2016).

Such ecological complexity of biosynthetic pathways is a common occurrence. It is quite possible that phosphine production requires a similarly complex ecological scenario, where the full biosynthetic pathway for $\mathrm{PH}_{3}$ synthesis is shared between multiple species of anaerobic microorganisms and only leads to phosphine formation under very specific environmental conditions (Bains et al., 2019a). Such complex ecological scenarios further complicate the elucidation of the direct mechanism of biosynthesis. Recent work postulated that $\mathrm{PH}_{3}$ production may be associated with the microbial tricarboxylic acid cycle of Enterobacter (Fan et al., 2020). As it is in the case of the complex ecology of biosynthesis of truffle volatiles, the lack of understanding of the details of their biological production does not mean that phosphine or any other natural molecule is not a product of the metabolism of the living organisms.

\section{Phosphines in Earth's Ecology}

All life on Earth relies on phosphorous-containing compounds in its metabolism. The great majority of biochemicals used by life on Earth are pentavalent phosphorus-containing molecules, predominantly phosphates (Petkowski et al., 2019b). At first glance, trivalent phosphorus compounds appear to be almost completely absent from biology, yet strong circumstantial evidence seems to suggest that anaerobic life on Earth explores chemistry of phosphines beyond simple phosphine production. One isolated study (Davies, 2008) reports identification of phospholane volatile in biological samples. Phospholane is a volatile, trivalent phosphoruscontaining, five-membered ring saturated hydrocarbon. It is the only molecule reported so far to be isolated from biological samples, which contains a bond between trivalent phosphorus and carbon atoms. Phospholane, like $\mathrm{PH}_{3}$, is a volatile trivalent phosphorus molecule that was identified in European 
badger fresh scat samples (Davies, 2008). It is interesting to note that, so far, all trivalent phosphorus-containing molecules that were isolated from biological samples (i.e., $\mathrm{PH}_{3}$ and phospholane) appear to be produced in strictly anoxic environments and appear to be exclusively associated with anaerobic $\mathrm{O}_{2}$-free dwelling microorganisms, which seems to be in agreement with previous observations that phosphines tend to be poisonous to aerobic life, but nontoxic in anoxic environments.

Both phosphine and phospholane were detected in anaerobic environments, which are generally difficult to study. If natural production of trivalent phosphorus-containing natural chemicals is strictly dependent on anoxic environments, it is likely that more such molecules are going to be discovered in the future. Almost all natural compounds reported to be produced by life on Earth were detected or isolated from organisms living in oxygenic environments, as shown by the repositories of natural molecules produced by life (Bains et al., 2019b; Petkowski et al., 2019a); thus, the small number of known natural molecules containing trivalent phosphorus might be the result of sample bias toward $\mathrm{O}_{2}$-rich environment. For a detailed study on the chemistry of $\mathrm{PH}_{3}$ in the context of the terrestrial biology, see Bains et al. (2019b).

\section{The Phosphorus Cycle on Earth}

It is generally assumed that life on Earth only consumes phosphorus in its most oxidized form of phosphate and that phosphates are the only form of phosphorus that is useful for life's cellular metabolism. This assumption is incorrect. Organisms can also use phosphorus in many other forms beyond phosphates, such as organic phosphorus esters, polyphosphates, phosphonates, and even reduced phosphorus species such as phosphites. Phosphite and hypophosphite biochemistry is well known (Casida Jr. 1960; Metcalf and Wolfe, 1998; Stone and White, 2012; Pasek et al., 2014; Figueroa and Coates, 2017), and some microorganisms living in strictly anoxic environments, such as anaerobic sludges in wastewater treatment plants, can fulfill all their energy requirements by oxidizing phosphite to phosphate (a process called dissimilatory phosphite oxidation) (Figueroa and Coates, 2017; Figueroa et al., 2018). Studies in recent years established that a complex redox phosphorus cycle (beyond the simple cycling of phosphates) has equally important bioenergetic and ecological consequences as the more recognized microbial nitrogen, carbon, or sulfur cycles (Karl, 2014).

Marine organisms can reduce a significant fraction (up to $15 \%$ ) of the total phosphates taken up from the surrounding environment (Van Mooy et al., 2015). The reduced phosphorus species (e.g., in the form of the soluble phosphite) are then rapidly released to the surrounding seawater (Van Mooy et al., 2015). The authors argue that the total amount of phosphorus cycled through such complex redox reactions could exceed other oceanic sources of phosphorus (e.g., direct continental and atmospheric sources) (Van Mooy et al., 2015). It is unknown why marine planktonic organisms engage in such an energetically costly endeavor. Production and rapid release of large quantities of reduced phosphorus species is unexpected, especially in the open oceanic waters where phosphorus is a limiting nutrient. The production of reduced phosphorus species is, of course, dependent on the abundance of phosphorus but appears to be independent of inorganic or organic phosphorus concentrations and independent of the overall phosphate turnover time (Van Mooy et al., 2015). The synthesis and rapid export of soluble reduced phosphorus species could stem from the general cooperative behavior of planktonic organisms in the open ocean. One of the more studied producers of reduced phosphorus species is the cyanobacteria Trichodesmium. The colonies of Trichodesmium are also inhabited by many species of epibiotic and symbiotic bacteria that appear to cooperate in the cycling of phosphorus (Hmelo et al., 2012; Van Mooy et al., 2012). Phosphonates and reduced phosphorus compounds (i.e., phosphites) may be part of a barter economy where phosphorus is exchanged for other nutrients between Trichodesmium and other species living in the cooperative colonies. Such examples of biological importance of rare redox phosphorus species clearly show that reduced phosphorus species play an important albeit underappreciated role in the global biogeochemical cycle of phosphorus.

The exact place and role of phosphine in the global phosphorus cycle is not yet fully known. It is, however, likely that, similar to other reduced phosphorus species, $\mathrm{PH}_{3}$ also has an important role in the global cycling of this essential element.

\section{Appendix References}

Amend, J.P. and Shock, E.L. (2001) Energetics of overall metabolic reactions of thermophilic and hyperthermophilic Archaea and Bacteria. FEMS Microbiol Rev 25:175-243.

Bains, W., Petkowski, J.J., and Seager, S. (2017) Toward a list of molecules as potential biosignature gases for the search for life on exoplanets: thermodynamic profiling potential false positives. In: Astrobiology Science Conference (AbSciCon), Mesa, Arizona.

Bains, W., Petkowski, J.J., Sousa-Silva, C., and Seager, S. (2019a) New environmental model for thermodynamic ecology of biological phosphine production. Sci Total Environ 658:521-536.

Bains, W., Petkowski, J.J., Sousa-Silva, C., and Seager, S. (2019b) Trivalent phosphorus and phosphines as components of biochemistry in anoxic environments. Astrobiology 19:885-902.

Barner, H.E. and Scheurman, R.V. (1978) Handbook of Thermochemical Data for Compounds and Aqueous Species. Wiley Interscience, New York, NY.

Bond, E.J. and Monro, H.A.U. (1967) The role of oxygen in the toxicity of fumigants to insects. J Stored Prod Res 3: 295-310.

Casida Jr., L. (1960) Microbial oxidation and utilization of orthophosphite during growth. J Bacteriol 80:237.

Conrad, R., Schink, B., and Phelps, T.J. (1986) Thermodynamics of $\mathrm{H} 2$-consuming and $\mathrm{H} 2$-producing metabolic reactions in diverse methanogenic environments under in situ conditions. FEMS Microbiol Lett 38:353-360.

Davies, M.J. (2008) Chemical Communication in the European Otter, Lutra lutra. PhD thesis, University of Hull.

Fan, Y., et al. (2020) Evidence and mechanism of biological formation of phosphine from the perspective of the tricarboxylic acid cycle. Int Biodeterior Biodegrad 146:104791.

Figueroa, I.A., and Coates, J.D. (2017) Chapter four-microbial phosphite oxidation and its potential role in the global phosphorus and carbon cycles. In: Advances in Applied Microbiology, edited by S. Sariaslani and G.M. Gadds, Academic Press, pp. 93-117. Available online at https://www .sciencedirect.com/science/article/pii/S0065216416301137 
Figueroa, I.A., Barnum, T.P., Somasekhar, P.Y., Carlström, C.I., Engelbrektson, A.L., and Coates, J.D. (2018) Metagenomicsguided analysis of microbial chemolithoautotrophic phosphite oxidation yields evidence of a seventh natural $\mathrm{CO}_{2}$ fixation pathway. Proc Natl Acad Sci U S A 115:E92-E101.

Fu, M., Yu, Z., Lu, G., and Song, X. (2013) Henry's law constant for phosphine in seawater: determination and assessment of influencing factors. Chin J Oceanol Limnol 31:860-866.

Gokhale, S.D., Jolly, W.L., Thomas, S., and Britton, D. (2007) Phosphine. In: Inorganic Syntheses, edited by S.Y. Tyrees, Jr. Copyright (C) 1967 by McGraw-Hill Book Company, Inc. Available online at https://onlinelibrary.wiley.com/doi/abs/ 10.1002/9780470132401.ch17

Hill, C., Yurchenko, S.N., and Tennyson, J. (2013) Temperaturedependent molecular absorption cross sections for exoplanets and other atmospheres. Icarus 226:1673-1677.

Hmelo, L.R., Van Mooy, B.A.S., and Mincer, T.J. (2012) Characterization of bacterial epibionts on the cyanobacterium Trichodesmium. Aquat Microb Ecol 67:1-14.

Hu, R., Seager, S., and Bains, W. (2012) Photochemistry in terrestrial exoplanet atmospheres. I. Photochemistry model and benchmark cases. Astrophys J 761:166.

Karl, D.M. (2014) Microbially mediated transformations of phosphorus in the sea: new views of an old cycle. Annu Rev Mar Sci 6:279-337.

Linstrom, P.J., and Mallard, W.G. (2001) The NIST Chemistry WebBook: a chemical data resource on the Internet. J Chem Eng Data 46:1059-1063.

Martin, W., Baross, J., Kelley, D., and Russell, M.J. (2008) Hydrothermal vents and the origin of life. Nat Rev Microbiol 6:805.

Metcalf, W.W. and Wolfe, R.S. (1998) Molecular genetic analysis of phosphite and hypophosphite oxidation by Pseudomonas stutzeri WM88. J Bacteriol 180:5547-5558.

Pasek, M.A., Sampson, J.M., and Atlas, Z. (2014) Redox chemistry in the phosphorus biogeochemical cycle. Proc Natl Acad Sci U S A 111:15468-15473.

Perkins, M.W., Wong, B., Olivera, D., and Sciuto, A. (2015) Phosphine. In: Hamilton \& Hardy's Industrial Toxicology, edited by M.W. Perkins, B. Wong, D. Olivera, and
A. Sciuto; Wiley Online Library, pp 1295-1300, Chapter 118. Available online at https://onlinelibrary.wiley.com/ doi/abs/10.1002/9781118834015.ch118

Petkowski, J.J., Bains, W., and Seager, S. (2018) Natural products containing a nitrogen-sulfur bond. J Nat Prod 81: 423-446.

Petkowski, J.J., Bains, W., and Seager, S. (2019a) An apparent binary choice in biochemistry: mutual reactivity implies life chooses thiols or nitrogen-sulfur bonds, but not both. Astrobiology 19:579-613.

Petkowski, J.J., Bains, W., and Seager, S. (2019b) Natural products containing 'rare' organophosphorus functional groups. Molecules 24:866.

Roels, J. and Verstraete, W. (2001) Biological formation of volatile phosphorus compounds. Bioresour Technol 79:243-250.

Seager, S., Bains, W., and Hu, R. (2013) Biosignature gases in H2-dominated atmospheres on rocky exoplanets. Astrophys $J$ 777:95.

Stone, B.L. and White, A.K. (2012) Most probable number quantification of hypophosphite and phosphite oxidizing bacteria in natural aquatic and terrestrial environments. Arch Microbiol 194:223-228.

Valmas, N., Zuryn, S., and Ebert, P.R. (2008) Mitochondrial uncouplers act synergistically with the fumigant phosphine to disrupt mitochondrial membrane potential and cause cell death. Toxicology 252:33-39.

Van Mooy, B.A.S., Hmelo, L.R., Sofen, L.E., Campagna, S.R., May, A.L., Dyhrman, S.T., Heithoff, A., Webb, E.A., Momper, L., and Mincer, T.J. (2012) Quorum sensing control of phosphorus acquisition in Trichodesmium consortia. ISME J 6:422.

Van Mooy, B.A.S., Krupke, A., Dyhrman, S.T., Fredricks, H.F., Frischkorn, K.R., Ossolinski, J.E., Repeta, D.J., Rouco, M., Seewald, J.D., and Sylva, S.P. (2015) Major role of planktonic phosphate reduction in the marine phosphorus redox cycle. Science 348:783-785.

Zambonelli, A., Iotti, M., and Murat, C., editors (2016) True Truffle (Tuber spp.) in the World: Soil Ecology, Systematics and Biochemistry. Springer International, Publishing Switzerland, Springer. 